Atratores para equações de ondas em domínios de fronteira móvel

\author{
Christian Manuel Surco Chuño
}



SERVIÇO DE PÓS-GRADUAÇÃO DO ICMC-USP

Data de Depósito:

Assinatura:

\section{Atratores para equações de ondas em domínios de fronteira móvel ${ }^{1}$}

\section{Christian Manuel Surco Chuño}

Orientador: Prof. Dr. Ma To Fu

Tese apresentada ao Instituto de Ciências Matemáticas e de Computação - ICMC-USP, como parte dos requisitos para obtenção do título de Doutor em Ciências - Matemática. VERSÃO REVISADA.

USP - São Carlos

Novembro de 2014

\footnotetext{
${ }^{1}$ Este trabalho teve apoio financeiro da CAPES de 01/09/2009 à 31/08/2013
} 
Ficha catalográfica elaborada pela Biblioteca Prof. Achille Bassi e Seção Técnica de Informática, ICMC/USP, com os dados fornecidos pelo(a) autor(a)

Surco Chuño, Christian Manuel
Atratores para equações de ondas em domínios de
fronteira móvel / Christian Manuel Surco Chuño;
orientador Ma To Fu . -- São Carlos, 2014 .
$80 \mathrm{p.}$
Tese (Doutorado - Programa de Pós-Graduação em
Matemática) -- Instituto de Ciências Matemáticas e
de Computação, Universidade de São Paulo, 2014.
1. Atratores pullback. 2. Equação de onda. 3.
Domínio não cilíndrico. I. Ma To Fu, orient. II.
Título.


A luta é a minha vida. Continuarei a luta pela liberdade até o fim de meus dias.

Nelson Mandela 


\section{Agradecimentos}

O principal agradecimento vai para minha mãe, pelo apoio e pela paz nos momentos em que me encontrei incapaz de prosseguir.

A meu pãe, irmãos, demais familiares e amigos que sempre me incentivaram e torceram pela minha vitória.

Ao estimado Dr. Ma To Fu, que considero um excelente professor e orientador e, acima de tudo, um grande amigo, sempre auxiliando de uma forma bastante criativa nas incontáveis dúvidas que surgiram durante a realização deste trabalho.

Aos amigos Eber, Napoleón, Lizandro, Manuel, José Bravo, Nancy, Luis, Nathaly, Eduar, Gian-

franco, Vanessa, Alessandra, José Alvites, Henry, Cecilia, Daniel, Luis Enrique, Irma e aos demais colegas do Programa de Pós-graduação em Matemática que me ajudaram no desenvolvimento desta tese.

À Coordenação de Aperfeiçoamento de Pessoal de Nível Superior (CAPES) pela bolsa de estudos de Doutorado.

Aos demais professores do Programa de Pós-graduação em Matemática, aos técnicos e demais profissionais do ICMC.

Tese de Doutorado dedicada a minha mãe Alejandra. 


\section{Resumo}

Este trabalho contém um estudo sobre equações de ondas fracamente dissipativas definidas em domínios de fronteira móvel

$$
\frac{\partial^{2} u}{\partial t^{2}}+\eta \frac{\partial u}{\partial t}-\Delta u+g(u)=f(x, t), \quad(x, t) \in \widehat{\mathscr{Q}}_{\tau}
$$

onde

$$
\widehat{\mathscr{Q}}_{\tau}=\bigcup_{t \in(\tau,+\infty)} \mathscr{O}_{t} \times\{t\}
$$

Dizemos que domínio $\widehat{\mathscr{Q}}_{\tau}$ possui fronteira móvel se admitirmos que a fronteira $\Gamma_{t}$ de $\mathscr{O}_{t}$ varia em relação a $t$. Nossa contribuição é dividida em três etapas. 1 - Provamos que o problema munido da condição de fronteira de Dirichlet é bem posto no sentido de Hadamard (existência global, unicidade e dependência contínua dos dados) para soluções fortes e fracas. Nessa etapa utilizamos um método clássico que transforma o domínio dependente de $t$ em um domínio fixo. Como consequência observamos que o sistema é essencialmente não autônomo. 2 - Buscamos uma teoria de sistemas dinâmicos não autônomos para estudar o operador solução do problema como um processo

$$
U(t, \tau): X_{\tau} \longrightarrow X_{t}, \quad t \geq \tau
$$

definido em espaços de fase $X_{t}=H_{0}^{1}\left(\mathscr{O}_{t}\right) \times L^{2}\left(\mathscr{O}_{t}\right)$ que são dependentes do tempo $t .3$ - No contexto da dinâmica de longo prazo encontramos hipóteses para garantir que o sistema dinâmico associado ao problema de ondas em domínios de fronteira móvel possui um atrator pullback. Basicamente admitimos que o domínio é crescente e "time-like". Salientamos que o nosso trabalho é o primeiro que estuda tais equações de ondas sob o ponto de vista de sistemas dinâmicos não-autônomos. Para equações parabólicas, resultados no mesmo contexto foram obtidos anteriormente por Kloeden, Marín-Rubio e Real [JDE 244 (2008) 2062-2090] e Kloeden, Real e Sun [JDE 246 (2009) 4702-4730]. Entretanto o nosso problema é hiperbólico e não possui a regularidade das equações parabólicas. 


\begin{abstract}
In this work we study a weakly dissipative wave equation defined in domains with moving boundary

$$
\frac{\partial^{2} u}{\partial t^{2}}+\eta \frac{\partial u}{\partial t}-\Delta u+g(u)=f(x, t), \quad(x, t) \in \widehat{\mathscr{Q}}_{\tau},
$$
\end{abstract}

where

$$
\widehat{\mathscr{Q}}_{\tau}=\bigcup_{t \in(\tau,+\infty)} \mathscr{O}_{t} \times\{t\}
$$

We says that a domain $\widehat{\mathscr{Q}}_{\tau}$ has moving boundary if the boundary $\Gamma_{t}$ of $\mathscr{O}_{t}$ varies with respect to $t$. Our contribution is threefold. 1 - We prove that the wave equation equipped with Dirichlet boundary condition is well-posed in the sense of Hadamard (global existence, uniqueness and continuous dependence with respect to data) for weak and strong solutions. This is done by using a classical argument that transforms the time dependent domain in a fixed domain. As a consequence we see that the problem is essentially non-autonomous. 2 - We find a theory of non-autonomous dynamical systems in order to study the solution operator as a process

$$
U(t, \tau): X_{\tau} \longrightarrow X_{t}, \quad t \geq \tau
$$

defined in time dependent phase spaces $X_{t}=H_{0}^{1}\left(\mathscr{O}_{t}\right) \times L^{2}\left(\mathscr{O}_{t}\right)$. 3 - In the context of long-time behavior of solutions we find suitable conditions to guarantee the existence of a pullback attractor. Roughly speaking, we assume the domain $Q$ is expanding and time-like. We emphasize that our work is the first one that consider wave equations in noncylindrical domains as non-autonomous dynamical systems. With respect to parabolic equations, similar results were early obtained by Kloeden, Marín-Rubio and Real [JDE 244 (2008) 2062-2090] and Kloeden, Real and Sun [JDE 246 (2009) 4702-4730]. However our problem is hyperbolic and does not enjoy regularity properties as the parabolic ones. 


\section{Sumário}

$\begin{array}{ll}\text { Introdução } & 1\end{array}$

1 Preliminares $\quad 5$

1.1 Principais resultados utilizados . . . . . . . . . . . . . 5

1.2 Espaços de Sobolev . . . . . . . . . . . . . . . . . . . 7

1.2.1 Os espaços $L^{p}(\Omega) \quad \ldots \ldots \ldots \ldots \ldots$

1.2.2 Os espaços $W^{m, p}(\Omega) \ldots \ldots \ldots \ldots \ldots$

1.3 Os espaços $L^{p}(\tau, T ; X) \ldots \ldots \ldots \ldots \ldots \ldots \ldots \ldots$

1.4 Processos de evolução e atratores pullback . . . . . . . . . . . . . . . . . 14

1.4.1 Definições básicas ......................... 14

1.5 Bases de atração e atratores $\mathscr{D}$-pullback . . . . . . . . . . . . . . . 22

2 Existência e unicidade de solução fraca para o problema de onda semilinear 27

2.1 Existência e unicidade de solução forte para o sistema auxiliar (2.2) . . . . . . . 28

2.1.1 Existência de solução forte para o sistema (2.3) . . . . . . . . . . . 30

2.1.2 Existência e unicidade de solução forte para o sistema (2.2) . . . . . . . . 39

2.2 Existência e unicidade de solução fraca para o sistema auxiliar (2.2) . . . . . . . . 42 
2.2.1 Existência de solução fraca para o sistema auxiliar (2.2) . . . . . . . . . 43

3 Processo de evolução associado ao problema de onda semilinear 49

3.1 Definição do processo correspondente . . . . . . . . . . . . . . . . 49

4 Atrator pullback para o problema de onda semilinear $\quad 54$

4.1 Atratores pullback ............................ 54

4.2 Resultado principal . . . . . . . . . . . . . . . . . 60

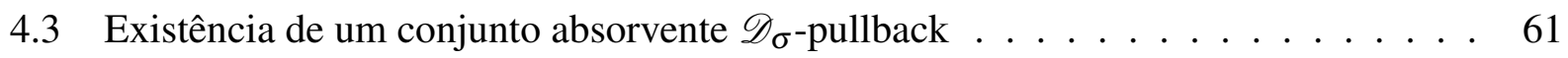

4.4 Compacidade assintótica $\mathscr{D}_{\sigma \text {-pullback } \ldots \ldots \ldots} \ldots \ldots$

Referências Bibliográficas 


\section{Introdução}

A presente tese contém um primeiro estudo da dinâmica a longo prazo (long-time behaviour) de equações de ondas definidas em domínios com fronteira móvel. Como veremos, tais problemas definem sistemas dinâmicos de dimensão infinita não autônomos. Nosso principal resultado é um teorema de existência de atratores pullback (Teorema 4.2.1).

\section{A equação de ondas em domínios com fronteira móvel.}

A classe de equações de ondas definidas em domínios de fronteira móvel pode ser apresentada da seguinte forma. Seja $\left\{\mathscr{O}_{t}\right\}_{t \in \mathbb{R}}$ uma família de abertos limitados de $\mathbb{R}^{N}$ cuja fronteira $\partial \mathscr{O}_{t}$ pode variar (continuamente) com relação ao parâmetro temporal $t$. Então podemos definir a equação de ondas

$$
\begin{aligned}
& u_{t t}-\Delta u+\eta u_{t}+g(u)=f(x, t), \quad x \in \mathscr{O}_{t}, t \geq \tau \\
& u(x, t)=0, \quad x \in \partial \mathscr{O}_{t}, t \geq \tau, \\
& u(x, \tau)=u_{0 \tau}(x), \quad u_{t}(x, \tau)=u_{1 \tau}(x), \quad x \in \mathscr{O}_{\tau},
\end{aligned}
$$

onde $\tau \in \mathbb{R}$ é um tempo inicial. Assim o domínio da variável independente $(x, t)$ é definido por

$$
\widehat{\mathscr{Q}}_{\tau}=\bigcup_{t \in(\tau,+\infty)} \mathscr{O}_{t} \times\{t\}
$$

e possui fronteira lateral

$$
\hat{\Sigma}_{\tau}=\bigcup_{t \in(\tau,+\infty)} \partial \mathscr{O}_{t} \times\{t\}
$$

Como as regiões $\left\{\mathscr{O}_{t}\right\}$ podem variar em relação a $t$, também dizemos que o domínio $\widehat{\mathscr{Q}}_{\tau}$ é nãocilíndrico. 
Tais equações são modelos matemáticos para diversos problemas físicos. Na literatura, os principais resultados pertinentes são dirigidos ao estudo da existência de soluções e ao comportamento assintótico das soluções. Resultados importantes para o nosso estudo se encontram em Lions [14], Cooper e Bardos [9] e Bardos e Chen [1].

Uma técnica para se estudar a existência de soluções desses problemas é supor que exista um difeomorfismo que transforma o domínio não cilíndrico $\widehat{\mathscr{Q}}_{\tau}$ em um domínio cilíndrico

$$
\mathscr{Q}_{\tau}=\mathscr{O} \times\{\tau\}
$$

Suponhamos que exista uma função $h \in C^{2}(\mathbb{R})$, inversível, tal que quando $(x, t)$ varia em $\widehat{\mathscr{Q}}_{\tau}$, o ponto $\left(h^{-1}(t) x, t\right)$ varia no cilindro $\mathscr{Q}_{\tau}$. Assim, definimos a aplicação

$$
r: \widehat{\mathscr{Q}}_{\tau} \rightarrow Q_{\tau}, \quad r(x, t)=(y, t)
$$

com $y=h^{-1}(t) x$. Tal aplicação é um difeomorfismo de classe $C^{2}$. Através dessa mudança de variáveis o problema original é transformado no problema de domínio fixo, fazendo $v(y, t)=$ $u(r(x, t))$,

$$
\begin{aligned}
& v_{t t}-A(t) v+a_{2} \cdot \nabla v_{t}+a_{3} \cdot \nabla v+\eta v_{t}+g(v)=f(y, t), \quad(y, t) \in Q_{\tau}, \\
& v(y, t)=0, \quad(y, t) \in \partial \mathscr{O} \times\{\tau\}, \\
& u(y, \tau)=v_{0 \tau}(y), \quad v_{t}(y, \tau)=v_{1 \tau}(y), \quad y \in \mathscr{O},
\end{aligned}
$$

onde

$$
A(t) v=-\sum_{k, l=1}^{N} \frac{\partial}{\partial y_{l}}\left(a_{k l}(y, t) \frac{\partial v}{\partial y_{k}}\right) .
$$

Os coeficientes $a_{2}=a_{2}(y, t), a_{3}=a_{3}(y, t)$ são vetores dependentes de $t$ e os coeficientes $a_{k l}$ são escalares.

Segue que a equação (1) a coeficientes constantes no domínio de fronteira móvel é equivalente a uma equação (2) de coeficientes dependentes de $t$ no domínio fixo, que é por definição, não autônomo. Dessa forma o estudo da dinâmica assintótica de equações de ondas em domínios de fronteira móvel conduz necessariamente ao estudo de sistemas dinâmicos não autônomos. 
Em condições apropriadas (ver Capítulo 2) provaremos que o problema (1) é bem posto e o operador solução corresponde a um processo não autônomo.

\section{Sistemas dinâmicos não autônomos e atratores pullback}

O estudo do comportamento a longo prazo de equações de evolução autônomas possui uma sólida fundamentação teórica baseado em semigrupos de operadores lineares e não-lineares. A teoria pode ser vista em, por exemplo, Babin e Vishik [24], Hale [26], Ladyzhenskaya [27] e Temam [28], entre outros. Por outro lado, a teoria da dinâmica de equações de evolução não autônomas é mais recente. Uma moderna e ampla apresentação da teoria pode ser vista em Carvalho, Langa e Robinson [6].

Dada um espaço métrico completo $(X, d)$ dizemos que uma família de operadores a dois parâmetros

$$
\{U(t, \tau)\}_{t \geq \tau}, \quad U(t, \tau): X \rightarrow X
$$

é um processo de evolução em $X$ se satisfaz as propriedades:

1. $U(t, t)=I$, para todo $t \in \mathbb{R}$,

2. $U(t, s)=U(t, \tau) U(\tau, s)$, para todo $t \geq \tau \geq s$,

3. $\left\{(t, s) \in \mathbb{R}^{2}: t \geq s\right\} \times X \ni(t, s, x) \longmapsto U(t, s) x \in X$ é contínuo.

Dado $t \in \mathbb{R}$, dizemos que um conjunto $B(t) \subset X$ atrai pullback um conjunto $D$ de $X$ no instante $t$ (sob a ação do processo $\{U(t, s): t \geq s\})$ se $\lim _{s \rightarrow-\infty} \operatorname{dist}(U(t, s) D, B(t))=0$, onde dist denota a semidistância de Hausdorff $\operatorname{dist}(A, B)=\sup _{x \in A} \inf _{y \in B} d(x, y)$ para $A, B \subset X$. Podemos então definir o atrator pullback para um processo de evolução. Dizemos que uma família $\mathscr{A}=\{\mathscr{A}(t)\}_{t \in \mathbb{R}}$ de conjuntos compactos é um atractor a pullback de $\{U(t, s)\}_{t \geq s}$ se

(i) $\mathscr{A}$ é invariant, i.e., $U(t, s) \mathscr{A}(s)=\mathscr{A}(t), \quad \forall t \geqslant s$.

(ii) $\mathscr{A}$ atrai pullback qualquer conjuntos limitados $B \subset X$,

(iii) $\mathscr{A}$ é a menor família de fechados que satisfaz (ii). 
Mas com relação ao nosso problema (1) o operador solução associa dados iniciais

$$
\left(u_{0 \tau}, u_{1 \tau}\right) \in X_{\tau}:=H_{0}^{1}\left(\mathscr{O}_{\tau}\right) \times L^{2}\left(\mathscr{O}_{\tau}\right)
$$

a uma solução (no instante $t \geq \tau$ )

$$
\left(u(\cdot, t), u_{t}(\cdot, t)\right) \in X_{t}:=H_{0}^{1}\left(\mathscr{O}_{t}\right) \times L^{2}\left(\mathscr{O}_{t}\right) .
$$

Então, em vista da unicidade de soluções e dependência contínua em relação aos dados, concluímos que o operador solução satisfaz as propriedades de processo de evolução $U(t, \tau)$ sobre espaços $X$, $t$-dependentes (não são mais fixos). Ou seja,

$$
\{U(t, \tau)\}_{t \geq \tau}, \quad U(t, \tau): X_{\tau} \rightarrow X_{t}
$$

Resulta que precisamos buscar uma teoria de sistemas dinâmicos não autônomos adequada para processos definidos em espações dependente de $t$.

\section{Estado da arte e Metodologia.}

Dois estudos da dinâmica a longo prazo de problemas de evolução em domínios de fronteira móvel foi recentemente apresentados por Kloeden, Marín-Rubio e Real [12] e Kloeden, Real e Sun [13]. No primeiro trabalho eles estudam a existência de atratores pullback para uma equação do calor (parabólica) através de um método de penalização. No segundo trabalho os autores também discutem a existência de atratores pullback para uma equação do calor. Entretanto empregam o método de mudança de variável. Além disso descrevem como trabalhar com processos do tipo $U(t, \tau): X_{\tau} \rightarrow X_{t}$. Nosso trabalho é fortemente baseado nos argumentos de [13].

Nossa contribuição é sobre equações de ondas em domínios não cilíndricos. Como a equação de onda fracamente dissipativa é hiperbólica, não satisfaz propriedades de regularidade da equação do calor. Dessa forma precisamos readequar vários argumentos para poder empregar os métodos em [13]. Em particular apresentamos um resultado novo sobre a compacidade assintótica do problema (Teorema 4.4.2). 


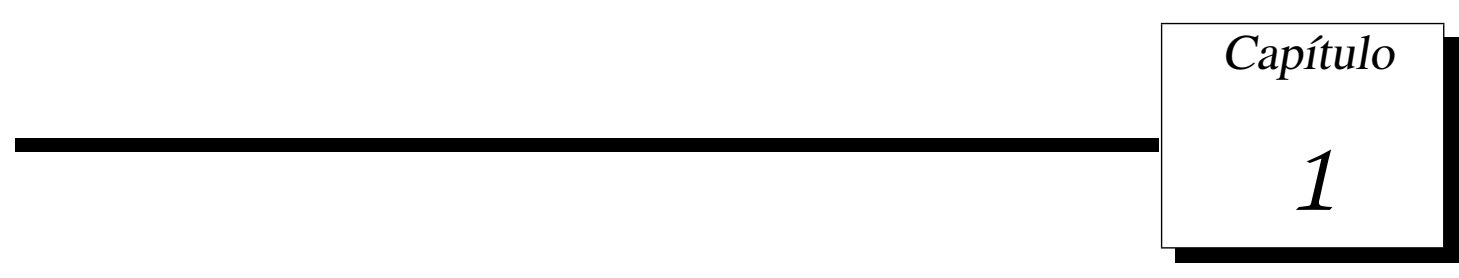

\section{Preliminares}

Neste capítulo apresentamos definições, notações e resultados, alguns adaptados da literatura, que foram fundamentais para o desenvolvimento da tese, uma vez que foram utilizadas em demostrações de teoremas importantes. Este capítulo está dividido em várias seções, onde cada uma trata de um tema específico.

\subsection{Principais resultados utilizados}

Seja $D$ um subconjunto do $\mathbb{R}^{N+1}$, cujos elementos são denotados por $(t, x)$, onde $t \in \mathbb{R}, x \in \mathbb{R}^{N}$. Considere $f: D \longrightarrow \mathbb{R}$ não necessariamente contínua. Consideramos a equação diferenciável 1.1, se existir uma função absolutamente contínua $x(t)$, definida em algum intervalo $I$ da reta, tal que $(t, x(t)) \in D$, para todo $t \in I \mathrm{e}$

$$
x^{\prime}(t)=f(t, x(t))
$$

para quase todo $t \in \mathbb{R}$, dizemos que $x(t)$ é uma solução de (1.1) sobre $I$. Se $\left(t_{0}, x_{0}\right) \in D$ está associado ao problema de valor inicial:

$$
\begin{aligned}
& x^{\prime}(t)=f(t, x) ; \\
& x\left(t_{0}\right)=x_{0},
\end{aligned}
$$

dizemos que a solução $x(t)$ de (1.1) sobre $I$, é tal que $t_{0} \in I$ e $x\left(t_{0}\right)=x_{0}$. 
Definição 1.1.1 (Condições de Carathéodory) Sejam D um subconjunto de $\mathbb{R}^{N+1}$ e $f: D \longrightarrow \mathbb{R}^{N}$. Então dizemos que $f$ satisfaz as condições de Carathéodory se:

i) $f(t, x)$ é mensurável em t para cada $x$ fixo;

ii) $f(t, x)$ é contínua em x para cada t fixo;

iii) Para cada compacto $U$ em $D$, existe uma função real integrável $m_{U}(t)$ tal que:

$$
|f(t, x)| \leq m_{U}(t), \text { para todo }(t, x) \in U
$$

Teorema 1.1.2 (Carathéodory) Seja $f: R \longrightarrow \mathbb{R}^{N}$ satisfazendo as condições de Carathéodory sobre $R$. Então existe uma solução $x(t)$ de (1.2) sobre algum intervalo $\left|t-t_{0}\right| \leq \beta, \beta>0$, onde $R$ é o retângulo definido por $R=\left\{(t, x) \in \mathbb{R}^{N+1}:\left|t-t_{0}\right| \leq a,\left|x-x_{0}\right| \leq b\right\}$, com $a>0, b>0$.

Demonstração: Ver [8].

Corolário 1.1.3 Sejam D um aberto do $\mathbb{R}^{N+1}$ e f satisfazendo as condições de Carathéodory sobre D. Então o problema (1.2) tem solução para qualquer $\left(t_{0}, x_{0}\right) \in D$.

Demonstração: Ver [8].

Teorema 1.1.4 (Banach-Alaoglu-Bourbaki) Seja $E$ um espaço de Banach. O conjunto $B_{E^{\prime}}=$ $\left\{f \in E^{\prime}:\|f\| \leq 1\right\}$ é compacto com respeito a topologia fraca estrela $* \sigma\left(E^{\prime}, E\right)$.

Demonstração: Ver [3].

Lema 1.1.5 (Desigualdade de Gronwall-Belman) Sejam $\tau, T \in \mathbb{R}, \tau<T$. Sejam $m \in L^{1}(\tau, T, \mathbb{R})$ tal que $m \geq 0$ q.t.p. em $(\tau, T)$ e a $\geq 0$ uma constante.

Seja $\phi:[\tau, T] \longrightarrow \mathbb{R}$ uma função contínua que verifica

$$
\phi(t) \leq a+\int_{\tau}^{t} m(s) \phi(s) d s, \quad \forall t \in[\tau, T] .
$$


Então

$$
\phi(t) \leq a e^{\int_{\tau}^{t} m(s) d s}, \forall t \in[\tau, T]
$$

Demonstração: Ver [2].

\subsection{Espaços de Sobolev}

\subsubsection{Os espaços $L^{p}(\Omega)$}

Dado $\Omega$ um aberto do $\mathbb{R}^{N}$, denotamos por $L^{p}(\Omega), 1 \leq p<\infty$, o espaço vetorial das (classes de) funções mensuráveis à Lebesgue $u: \Omega \longrightarrow \mathbb{R}$ tais que $|u|^{p}$ é integrável no sentido de Lebesgue em $\Omega$, equipado da norma:

$$
\|u\|_{L^{p}(\Omega)}=\left(\int_{\Omega}|u(x)|^{p} d x\right)^{1 / p}
$$

No caso $p=\infty$, denota-se por $L^{\infty}(\Omega)$, o espaço vetorial das (classes de) funções mensuráveis a Lebesgue e essencialmente limitadas em $\Omega$, isto é, existe uma constante $C>0$ tal que

$$
|u(x)| \leq C, \quad \text { quase sempre em } \quad \Omega
$$

onde quase sempre significa a menos de um conjunto de medida nula.

Neste espaço considera-se a seguinte norma

$$
\|u\|_{L^{\infty}(\Omega)}=\sup _{x \in \Omega} \operatorname{ess}|u(x)|
$$

O espaço $L^{p}(\Omega), 1 \leq p \leq \infty$, com sua respectiva norma é um espaço de Banach. Em particular, quando $p=2$, tem-se que $L^{2}(\Omega)$ é um espaço de Hilbert cuja norma e produto interno serão definidos e denotados, respectivamente por

$$
|u|=\|u\|_{L^{2}(\Omega)}=\left(\int_{\Omega}|u(x)|^{2} d x\right)^{1 / 2} \quad \mathrm{e} \quad(u, v)_{L^{2}(\Omega)}=\int_{\Omega} u(x) v(x) d x
$$


Lema 1.2.1 (Lema de Lions) Sejam $U$ um aberto limitado do $\mathbb{R}^{N},\left(g_{n}\right)_{n \in \mathbb{N}}$ e g funções de $L^{q}(U)$, $1 \leq q \leq \infty$, tais que:

$$
\left\|g_{m}\right\|_{L^{q}(U)} \leq \text { C e } g_{m} \longrightarrow g \text { quase sempre em } U \text {. }
$$

Então $g_{m} \longrightarrow g$ fracamente em $L^{q}(U)$.

Demonstração: Ver [16].

Lema 1.2.2 (Desigualdade de Young) Sejam $p, q>1$, tais que, $\frac{1}{p}+\frac{1}{q}=1$. Então

$$
a b \leq \frac{a^{p}}{p}+\frac{b^{q}}{q} \text { para todo } a, b \geq 0
$$

Demonstração: Ver [3]

Lema 1.2.3 (Desigüaldade de Hölder) Sejam $f \in L^{p}(\Omega)$ e $g \in L^{q}(\Omega)$, com $\frac{1}{p}+\frac{1}{q}=1$ e $1 \leq p, q \leq$ $\infty$. Então $f g \in L^{1}(\Omega) e$

$$
\int_{\Omega}|f g| \leq\|f\|_{L^{p}(\Omega)}\|f\|_{L^{q}(\Omega)}
$$

Demonstração: Ver [3].

Lema 1.2.4 (Du Bois Raymond) Seja $u \in L_{l o c}^{1}(\Omega)$. Então

$$
\int_{\Omega} u(x) \phi(x) d x=0, \text { para todo } \phi \in D(\Omega)
$$

se, o somente se, $u=0$ quase sempre em $\Omega$.

Demonstração: Ver [3].

Teorema 1.2.5 (Rellich) Seja $\Omega$ um aberto limitado de classe $C^{1}$. Então a imersão do $H^{1}(\Omega)$ no $L^{2}(\Omega)$ é compacta. 
Demonstração: Ver [3].

Representamos por $L_{l o c}^{q}(\Omega), 1 \leq q<\infty$, o espaço vetorial das (classes de) funções $u: \Omega \longrightarrow \mathbb{R}$ tais que $|u|^{q}$ é integrável sobre qualquer compacto $K$ de $\Omega$.

Como $L^{p}(\Omega) \subset L_{l o c}^{1}(\Omega)$, para todo $1 \leq p \leq \infty$, concluímos que toda função $u \in L^{p}(\Omega)$ pode ser identificada a uma distribuição por ela definida e temos a seguinte cadeia de injeções contínuas e densas:

$$
D(\Omega) \hookrightarrow L^{p}(\Omega) \hookrightarrow L_{l o c}^{1}(\Omega) \hookrightarrow D^{\prime}(\Omega), 1 \leq p \leq \infty .
$$

Temos que, se $u \in L^{p}(\Omega)$, então sua derivada, no sentido das distribuições, não pertence necessariamente a $L^{p}(\Omega)$.

\subsubsection{Os espaços $W^{m, p}(\Omega)$}

Seja $m>0$, um número inteiro positivo e $1 \leq p \leq \infty$. O espaço de Sobolev de ordem $m$, denotado por $W^{m, p}(\Omega)$, é por definição o espaço vetorial das (classes de) funções de $L^{p}(\Omega)$ para as quais suas derivadas até a ordem $\alpha$, no sentido das distribuições, pertencem a $L^{p}(\Omega)$, para todo multi-índice $\alpha, \operatorname{com}|\alpha| \leq m$. O espaço $W^{m, p}(\Omega)$ será equipado com norma

$$
\|u\|_{W^{m, p}(\Omega)}=\left(\sum_{|\alpha| \leq m}\left\|D^{\alpha} u\right\|_{L^{p}(\Omega)}^{p}\right)^{1 / p}, 1 \leq p<\infty
$$

e quando $p=\infty$, define-se

$$
\|u\|_{W^{m, \infty}(\Omega)}=\sum_{|\alpha| \leq m}\left\|D^{\alpha} u\right\|_{L^{\infty}(\Omega)}
$$

Proposição 1.2.6 Os espaços lineares $W^{m, p}(\Omega)$ equipados das respectivas normas acima são espaços de Banach.

Demonstração: Ver [3].

$\mathrm{O}$ espaço $W^{m, p}(\Omega)$ é um espaço reflexivo se $1<p<\infty$ e separável se $1 \leq p<\infty$. No caso particular em que $p=2$, o espaço $W^{m, 2}(\Omega)$ é um espaço de Hilbert, que é denotado por $H^{m}(\Omega)$. 
Simbolicamente

$$
H^{m}(\Omega)=\left\{u \in L^{2}(\Omega) ; D^{\alpha} u \in L^{2}(\Omega), \forall \alpha,|\alpha| \leq m\right\}
$$

cuja norma e produto interno são por

$$
\|u\|_{H^{m}(\Omega)}=\left(\sum_{|\alpha| \leq m}\left\|D^{\alpha} u\right\|_{L^{2}(\Omega)}^{2}\right)^{1 / 2} \text { e }(u, v)=\sum_{|\alpha| \leq m}\left(D^{\alpha} u, D^{\alpha} v\right)_{L^{2}(\Omega)}
$$

respectivamente.

O espaço $H^{m}(\Omega)$ com a estrutura topológica acima é um espaço de Hilbert continuamente imerso em $L^{2}(\Omega)$.

Define-se o espaço $W_{0}^{m, p}(\Omega)$ como sendo o fecho de $C_{0}^{\infty}(\Omega)$ em $W^{m, p}(\Omega)$, em particular se $p=2$ denotaremos $W_{0}^{m, 2}(\Omega)=H_{0}^{m}(\Omega)$.

O dual topológico do espaço $W_{0}^{m, p}(\Omega)$ é representado por $W^{-m, q}(\Omega)$ se $1 \leq p<\infty$ com $p$ e $q$ índices conjugados. Se $\varphi \in W^{-m, q}(\Omega)$ então $\left.\varphi\right|_{\mathscr{D}(\Omega)}$ pertence a $\mathscr{D}^{\prime}(\Omega)$. Quando $p=2$, $W_{0}^{m, 2}(\Omega)$ é denotado por $H_{0}^{m}(\Omega)$, cujo dual é o espaço denotado por $H^{-m}(\Omega)$. A caracterização de $W^{-m, p}(\Omega)$ é dada por:

Teorema 1.2.7 Seja $T \in \mathscr{D}^{\prime}(\Omega)$. Então, $T \in W^{-m, p}(\Omega)$ se, e somente se, existem $g_{\alpha} \in L^{q}(\Omega)$ tais que, $T=\sum_{|\alpha| \leq m} D^{\alpha} g_{\alpha}$.

Demonstração: Ver [3].

Lema 1.2.8 (Desigualdade de Poincaré) Seja $\Omega \subset \mathbb{R}^{N}$ um aberto limitado em alguma direção. Se $u \in H_{0}^{1}(\Omega)$, então existe uma constante $C>0$ tal que

$$
\|u\|_{L^{2}(\Omega)}^{2} \leq C\|\nabla u\|_{L^{2}(\Omega)}^{2}
$$

Demonstração: Ver [3].

Observação 1.2.9 Usando a desigualdade de Poincaré conclui-se que em $H_{0}^{1}(\Omega)$, as normas $\|u\|_{H^{1}(\Omega)} e\|\nabla u\|_{L^{2}(\Omega)}$ são equivalentes. 
Teorema 1.2.10 Seja $\Omega$ um subconjunto limitado do $\mathbb{R}^{n},(n \geq 2)$, com $\partial \Omega$ de Classe $C^{m} e 1 \leq p<$ $\infty$, então

a) $W^{m, p}(\Omega) \hookrightarrow L^{q}(\Omega), 1 \leq q \leq \frac{n p}{n-m p}$ se $m p<n$,

b) $W^{m, p}(\Omega) \hookrightarrow L^{q}(\Omega), 1 \leq q<\infty$ se $m p=n$,

c) $W^{m, p}(\Omega) \hookrightarrow C^{k, \lambda}(\bar{\Omega})$ se $m p>n$.

No caso c), $k$ é um inteiro verificando $k<m-\frac{n}{p} \leq k+1$ e $\lambda$ um real satisfazendo $0<\lambda \leq$ $m-k-\frac{n}{p}=\lambda_{0}$ se $\lambda_{0}<1$ e $0<\lambda<1$ se $\lambda_{0}=1$.

Demonstração: Ver [3].

Teorema 1.2.11 (Rellich-Kondrachov) Sejam $\Omega$ um aberto limitado do $\mathbb{R}^{n}$, com $\partial \Omega$ de classe $C^{1}$ $e 1 \leq p \leq \infty$. Então as seguintes imersões são compactas:

a) $W^{1, p}(\Omega) \hookrightarrow L^{q}(\Omega), 1 \leq q<\frac{n p}{n-p}$ se $p<n$,

b) $W^{1, p}(\Omega) \hookrightarrow L^{q}(\Omega), 1 \leq q<\infty$ se $p=n$,

c) $W^{1, p}(\Omega) \hookrightarrow C^{0}(\bar{\Omega})$ se $p>n$.

Demonstração: Ver [3].

\subsection{Os espaços $L^{p}(\tau, T ; X)$}

Sejam $X$ um espaço de Banach real com a norma $\|\cdot\|_{X}, \tau<T$ números reais e $\chi_{E}$ a função característica do conjunto $E$. Uma função vetorial $\varphi:(\tau, T) \longrightarrow X$, é dita simples quando assume apenas um número finito de valores distintos. Dada uma função simples $\varphi:(\tau, T) \longrightarrow X$ com representação canônica

$$
\varphi(t)=\sum_{i=1}^{k} \chi_{E_{i}} \varphi_{i}
$$

onde $E_{i} \subset(\tau, T)$ é mensurável, $i=1,2, \ldots, k$, dois a dois disjuntos, $m\left(E_{i}\right)<\infty$ e $\varphi_{i} \in X, i=1,2, \ldots, k$. Define-se a integral de $\varphi$ como sendo o vetor de $X$ dado por

$$
\int_{\tau}^{T} \varphi(t) d t=\sum_{i=1}^{k} m\left(E_{i}\right) \varphi_{i}
$$


Diz-se que uma função vetorial $u:(\tau, T) \longrightarrow X$ é Bochner integrável $(\mathscr{B}$-integrável $)$ se existir uma seqüência $\left(\varphi_{v}\right)_{v \in \mathbb{N}}$ de funções simples, tal que:

i) $\varphi_{v} \longrightarrow u$ em $X$, q.s. em $(\tau, T)$;

ii) $\lim _{k, m \rightarrow \infty} \int_{\tau}^{T}\left\|\varphi_{k}(t)-\varphi_{m}(t)\right\|_{X} d t=0$

Uma função vetorial $u:(\tau, T) \subset \mathbb{R} \longrightarrow X$ é fracamente mensurável quando a função numérica $t \mapsto\langle\Phi, u(t)\rangle$ for mensurável, $\forall \Phi \in X^{\prime}$, onde $X^{\prime}$ é o dual topológico de $X$. Diz-se que $u$ é fortemente mensurável quando $u$ for limite quase sempre de uma seqüência $\left(\varphi_{v}\right)_{v \in \mathbb{N}}$ de funções simples. Em particular, quando $u$ for fortemente mensurável, então a aplicação $t \mapsto\|u(t)\|_{X}$ é mensurável à Lebesgue.

Denota-se por $L^{p}(\tau, T ; X), 1 \leq p<\infty$, o espaço vetorial das classes de funções $u:(\tau, T) \longrightarrow X$ fortemente mensuráveis e tais que a função $t \mapsto\|u(t)\|_{X}^{p}$ é integrável à Lesbegue em $(\tau, T)$, munido da norma

$$
\|u\|_{L^{p}(\tau, T ; X)}=\left(\int_{\tau}^{T}\|u(t)\|_{X}^{p} d t\right)^{1 / p}
$$

Quando $p=2$ e $X=H$ é um espaço de Hilbert, o espaço $L^{2}(\tau, T ; H)$ é também um espaço de Hilbert cujo produto interno é dado por

$$
(u, v)_{L^{2}(\tau, T ; H)}=\int_{\tau}^{T}(u(s), v(s))_{H} d s
$$

Por $L^{\infty}(\tau, T ; X)$ representamos o espaço de Banach das (classes de) funções $u:(\tau, T) \subset \mathbb{R} \longrightarrow X$ que são fortemente mensuráveis e tais que $t \mapsto\|u(t)\|_{X} \in L^{\infty}(\tau, T)$. A norma em $L^{\infty}(\tau, T ; X)$ é definida por

$$
\|u\|_{L^{\infty}(\tau, T ; X)}=\sup _{t \in(\tau, T)} \operatorname{ess}\|u(t)\|_{X}
$$

Quando $X$ é reflexivo e separável e $1<p<\infty$, então $L^{p}(0, T ; X)$ é um espaço reflexivo e separável, cujo dual topológico se identifica ao espaço de Banach $L^{p^{\prime}}\left(\tau, T ; X^{\prime}\right)$, onde $p$ e $p^{\prime}$ são índices conjugados, isto é, $\frac{1}{p}+\frac{1}{p^{\prime}}=1$. Mais precisamente, mostra-se que para cada $u \in\left[L^{p}(\tau, T ; X)\right]^{\prime}$, 
existe $\widetilde{u} \in L^{p^{\prime}}\left(\tau, T ; X^{\prime}\right)$ tal que

$$
\langle u, \varphi\rangle_{\left(L^{p}(\tau, T ; X)\right)^{\prime} \times L^{p}(\tau, T ; X)}=\int_{\tau}^{T}\langle\widetilde{u}(t), \varphi(t)\rangle_{X^{\prime} \times X} d t .
$$

No caso, $p=1$, o dual topológico do espaço $L^{1}(\tau, T ; X)$ se identifica ao espaço $L^{\infty}\left(\tau, T ; X^{\prime}\right)$.

O espaço das aplicações lineares e contínuas de $\mathscr{D}(\tau, T)$ em $X$ é denominado espaço das distribuições vetoriais sobre $(\tau, T)$ com valores em $X$, o qual será denotado por $\mathscr{D}^{\prime}(\tau, T ; X)$.

Definição 1.3.1 Seja $T \in \mathscr{D}^{\prime}(\tau, T ; X)$. A derivada de ordem né definida como sendo a distribuição vetorial sobre $(\tau, T)$ com valores em $X$ dada por

$$
\left\langle\frac{d^{n} T}{d t^{n}}, \varphi\right\rangle=(-1)^{n}\left\langle T, \frac{d^{n} \varphi}{d t^{n}}\right\rangle, \forall \varphi \in \mathscr{D}^{\prime}(\tau, T)
$$

Por $C^{0}([\tau, T] ; X), \tau<T<\infty$ representamos o espaço de Banach das funções contínuas $u$ : $[\tau, T] \longrightarrow X$ munido da norma da convergência uniforme

$$
\|u\|_{C^{0}([\tau, T] ; X)}=\max _{t \in[\tau, T]}\|u(t)\|_{X}
$$

Por $C_{w}^{0}([\tau, T] ; X)$ denotamos o espaço das funções $u:[\tau, T] \longrightarrow X$ fracamente contínuas, isto é, a aplicação $t \mapsto\langle v, u(t)\rangle_{X^{\prime}, X}$ é contínua em $[\tau, T], \forall v \in X^{\prime}$.

Quando $X=H$ é um espaço de Hilbert, a continuidade fraca de $u$ é equivalente a continuidade da aplicação $t \longmapsto(u(t), v)_{H}$ para $\forall v \in H$.

Teorema 1.3.2 (Aubin-Lions) Sejam $B_{0}, B, B_{1}$ espaços de Banach, $B_{0}$ e $B_{1}$ reflexivos, a imersão de $B_{0}$ em $B$ é compacta, B imerso continuamente em $B_{1}, 1<p_{0}, p_{1}<\infty$, e, W o espaço

$$
W=\left\{u \in L^{p_{0}}\left(\tau, T ; B_{0}\right) ; u^{\prime} \in L^{p_{1}}\left(\tau, T ; B_{1}\right)\right\}
$$

equipado da norma $\|u\|_{W}=\|u\|_{L^{p_{0}\left(\tau, T ; B_{0}\right)}}+\left\|u^{\prime}\right\|_{L^{p_{1}\left(\tau, T ; B_{1}\right)}}$. Então $W$ é um espaço de Banach, e a imersão de $W$ em $L^{p_{0}}(\tau, T ; B)$ é compacta. 
Demonstração: Ver [16].

Observação 1.3.3 Uma conseqüência do Teorema de Aubin-Lions 1.3.2: se $\left(u_{v}\right)_{v \in \mathbb{N}}$ é uma seqüência limitada em $L^{2}\left(\tau, T ; B_{0}\right) e\left(u_{v}^{\prime}\right)_{v \in \mathbb{N}}$ é uma seqüência limitada em $L^{2}\left(\tau, T ; B_{1}\right)$ então $\left(u_{v}\right)_{v \in \mathbb{N}}$ é limitada em $W$. Daí, segue que existe uma subsequência $\left(u_{v_{k}}\right)_{k \in \mathbb{N}} d e\left(u_{v}\right)_{v \in \mathbb{N}}$ tal que $u_{v_{k}} \longrightarrow$ u forte em $L^{2}(\tau, T ; B)$.

Proposição 1.3.4 Sejam $V$ e $H$ espaços de Hilbert, $V$ continuamente imerso em $\mathrm{H}$, $u \in L^{p}(\tau, T ; V) e u^{\prime} \in L^{p}(\tau, T ; H), \operatorname{com} 1 \leq p<\infty$, então

$$
u \in C^{0}([\tau, T] ; H) \cap C_{w}^{0}([\tau, T] ; V)
$$

Demonstração: Ver [21].

\subsection{Processos de evolução e atratores pullback}

\subsubsection{Definições básicas}

Suponha que temos uma equação diferencial não-autônoma em um espaço de Banach $X$

$$
\begin{array}{r}
u_{t}=F(u(t), t), \\
u(s)=u_{0},
\end{array}
$$

com uma solução única $u\left(t, s, u_{0}\right)$.

Em geral, um sistema não-autônomo mostra duas importantes dinâmicas diferentes sem relação entre eles:

- Dinâmica Forward: o comportamento quando o tempo final $t$ vai para o infinito,

$$
\lim _{t \rightarrow \infty} u\left(t, s ; u_{0}\right) .
$$


- Dinâmica Pullback: o comportamento quando o tempo inicial $s$ vai para o infinito negativo,

$$
\lim _{s \rightarrow-\infty} u\left(t, s ; u_{0}\right) .
$$

No caso autonômo, estas duas dinâmicas são a mesma, mas em geral eles podem produzir propriedades qualitativas completamente diferentes.

Então, precisamos definir uma família de dois parâmetros em vez de uma família de um parâmetro como no caso autônomo para descrever a completa dinâmica do sistema.

Definição 1.4.1 Um processo de evolução em $X$ é uma família de transformações $\{S(t, s): t \geq s\}$ em $\mathscr{C}(X)$ com as seguintes propriedades

1. $S(t, t)=I$, para todo $t \in \mathbb{R}$,

2. $S(t, s)=S(t, \tau) S(\tau, s)$, para todo $t \geq \tau \geq s$,

3. $\left\{(t, s) \in \mathbb{R}^{2}: t \geq s\right\} \times X \ni(t, s, x) \longmapsto S(t, s) x \in X$ é contínua.

A transformação $S(t, s)$ toma cada estado $x$ do sistema no instante inicial $s$ e evoluciona para o estado $S(t, s) x$ do sistema no tempo final $t$. Observamos que, para um $\sigma \in \mathbb{R}$ fixo, o operador $S(\sigma+\tau, \tau)$ pode ser um operador distinto para cada valor de $\tau \in \mathbb{R}$. Isto indica que, além do tempo decorrido $\sigma$, o instante inicial $\tau$ também pode desempenhar um papel importante no processo de evolução. A classe dos processos para os quais o tempo decorrido determina a evolução; isto é, os processos de evolução para os quais $S(t, s)=S(t-s, 0)$, para todo $t \geq s$, são chamados processos de evolução autônomos.

Em equações diferenciais, os processos de evolução são os sistemas dinâmicos naturais a serem estudados enquanto que os particulares sistemas dinâmicos associados as equações diferenciais autônomas são os semigrupos.

Em um processo de evolução autônomo $\{S(t, s): t \geq s\}$, o comportamento das soluções quando $t \longrightarrow \infty$, conhecido como dinâmica forwards, é o mesmo que o comportamento das soluções quando $s \longrightarrow-\infty$, chamado dinâmica pullback. Para processos de evolução gerais, estes dois comporta- 
mentos assintóticos não estão relacionados e podem produzir propriedades qualitativas completamente distintas. Um dos nossos propósitos é revelar algumas dessas propriedades dinâmicas novas que a dinâmica pullback pode conter.

A seguir apresentaremos alguns exemplos que nos ajudarão a desenvolver uma noção adequada de atratores para processos de evolução.

Exemplos 1.4.2 Os seguintes exemplos foram compilados da referência [6]. Considere os problemas de valor inicial

$$
\begin{aligned}
& \mid \begin{array}{l}
x^{\prime}(t)=-\alpha x(t)+t \\
x(s)=x_{0} .
\end{array} \\
& y^{\prime}(t)=-\alpha y(t)+\operatorname{sen} t \\
& y(s)=y_{0} .
\end{aligned}
$$

Cujas soluções são

$$
\begin{gathered}
x\left(t, s ; x_{0}\right)=\left(x_{0}+\frac{1}{\alpha^{2}}-\frac{1}{\alpha} s\right) e^{-\alpha(t-s)}+\frac{1}{\alpha} t-\frac{1}{\alpha^{2}} \quad e \\
y\left(t, s ; y_{0}\right)=\left(y_{0}-\frac{1}{1+\alpha^{2}}[\alpha \operatorname{sen} s-\cos s]\right) e^{-\alpha(t-s)}+\frac{1}{1+\alpha^{2}}[\alpha \operatorname{sen} t-\cos t] .
\end{gathered}
$$

Se definimos $S_{1}(t, s): \mathbb{R} \longrightarrow \mathbb{R}, t \geq s$ e $S_{2}(t, s): \mathbb{R} \longrightarrow \mathbb{R}, t \geq s$, onde $S_{1}(t, s) x_{0}=x\left(t, s ; x_{0}\right) e$ $S_{2}(t, s) y_{0}=y\left(t, s ; y_{0}\right)$, e assim é claro que $\left\{S_{i}(t, s): t \geq s\right\}$ é um processo de evolução, $i=1,2$. Vamos tornar evidente a importância do instante inicial s no estudo das propriedades assintóticas das soluções dos processos de evolução $\left\{S_{i}(t, s): t \geq s\right\}, i=1,2$, dados acima.

Primeiramente, note que todas as soluções de (1.5) e (1.6) existem globalmente e tendem $a+\infty$ quando $t \longrightarrow+\infty$; isto é, todas as soluções são ilimitadas. Embora a presença do termo $-\alpha x(t)$ represente uma dissipação no sistema, a ideia de atração é perdida para este exemplo não dando qualquer informação sobre o comportamento assintótico do sistema. 
Por outro lado, o efeito da dissipação pode ser observado se subtraímos duas soluções diferentes $x_{1}(t), x_{2}(t)$ ou $y_{1}(t), y_{2}(t)$ que corresponde a dados iniciais $x_{0}^{1}, x_{0}^{2}$ ou $y_{0}^{1}, y_{0}^{2}$. De fato, se denotamos a diferença dessas duas soluções por $z_{1}(t), z_{2}(t)$, temos que

$$
\frac{d}{d t}\left(z_{1}(t)-z_{2}(t)\right)=-\alpha\left(z_{1}(t)-z_{2}(t)\right)
$$

e logo,

$$
z_{1}(t)-z_{2}(t)=e^{-\alpha(t-s)}\left(x_{0}^{1}-x_{0}^{2}\right)
$$

Isto significa que, embora todas as soluções tendem para $+\infty$ quando t tende a $+\infty$, elas se aproximam assintoticamente (uniformemente para dados iniciais em conjuntos limitados) da solução $x(t)=\frac{t}{\alpha}-\frac{1}{\alpha^{2}}$ ou $\frac{1}{1+\alpha^{2}}[\alpha \operatorname{sen} t-\cos t] d e(1.5)$ e (1.6).

Se consideramos as famílias

$$
\left\{\mathscr{A}_{1}(t)=\frac{1}{\alpha} t-\frac{1}{\alpha^{2}}\right\}_{t \in \mathbb{R}} \quad e \quad\left\{\mathscr{A}_{2}(t)=\frac{1}{1+\alpha^{2}}[\alpha \operatorname{sen} t-\cos t]\right\}_{t \in \mathbb{R}} .
$$

É claro que

1. $S_{1}(t, s) \mathscr{A}_{1}(s)=\mathscr{A}_{1}(t)$ e $S_{2}(t, s) \mathscr{A}_{2}(s)=\mathscr{A}_{2}(t) \quad$ para todo $t \geq s$, com $t, s \in \mathbb{R}$.

2. (Atração Forwards) Se $B \subset \mathbb{R}$ é limitado

$$
\begin{aligned}
& \lim _{t \rightarrow+\infty} \operatorname{dist}\left(S_{1}(t, s) B, \mathscr{A}_{1}(t)\right)=0 \\
& \lim _{t \rightarrow+\infty} \operatorname{dist}\left(S_{2}(t, s) B, \mathscr{A}_{2}(t)\right)=0 .
\end{aligned}
$$

Nestes exemplos parece razoável chamar as famílias $\left\{\mathscr{A}_{1}(t): t \in \mathbb{R}\right\} e\left\{\mathscr{A}_{2}(t): t \in \mathbb{R}\right\}$ de atratores globais para $\left\{S_{1}(t, s): t \geq s\right\}$ e $\left\{S_{2}(t, s): t \geq s\right\}$. Note que $\left\{\mathscr{A}_{1}(t): t \in \mathbb{R}\right\}$ é ilimitado enquanto que $\left\{\mathscr{A}_{2}(t): t \in \mathbb{R}\right\}$ está contida no intervalo $\left[-\left(1+\alpha^{2}\right)^{-\frac{1}{2}},\left(1+\alpha^{2}\right)^{-\frac{1}{2}}\right]$.

Em ambos exemplos não existe um subconjunto fixo $\mathscr{A}$ de $\mathbb{R}$, que é invariante e para o qual

$$
\lim _{s \rightarrow-\infty} \operatorname{dist}\left(S(t, s) x_{0}, \mathscr{A}\right)=0
$$


Poderíamos então pensar em definir atrator para um processo da seguinte maneira:

Uma família $\{\mathscr{A}(t) \subset: t \in \mathbb{R}\}$ é um atrator para o processo $\{S(t, s): t \geq s\}$ se é invariante, $\mathscr{A}(t) e ́$ compacto para cada $t \in \mathbb{R}$ e atrai subconjuntos limitados; isto é, para cada subconjunto limitado $B \subset X$ e $s \in \mathbb{R}, \lim _{t \rightarrow+\infty} \operatorname{dist}(S(t, s) B, \mathscr{A}(t))=0$. Infelizmente tal definição somente seria adequada para uma pequena classe de processos com propriedades bastante específicas.

Para ver isto considere o seguinte exemplo

$$
x^{\prime}(t)=h(t) x-x^{3}
$$

onde $h: \mathbb{R} \longrightarrow[0,1]$ é uma função continuamente diferenciável que é igual a 0 para $t \leq 0$ e igual a 1 parat $\geq 1$.

Este exemplo simples (que deveria ter um atrator) não tem atrator no sentido da definição acima. Essencialmente, isto se deve ao fato que a maioria da dinâmica assintótica forwards está, neste caso, associada a soluções que explodem em tempo finito para trás.

Continuando a interpretar nossos exemplos, observamos que as famílias $\left\{\mathscr{A}_{i}(t)\right\}, i=1,2$, são os limites pullback (isto é, quando $s \longrightarrow-\infty$ ) de toda solução de (1.3), (1.4) e é uniforme para $x_{0}$ em conjuntos limitados de $\mathbb{R}$; isto é,

$$
\lim _{s \rightarrow-\infty} \sup _{x_{0} \in B} \operatorname{dist}\left(S(t, s) x_{0}, \mathscr{A}_{i}(t)\right)=0 .
$$

Este limite pullback determina $\mathscr{A}_{i}(t), i=1,2$, para todo $t \in \mathbb{R} e$ da origem a objetos com propriedades dinâmicas. Este fato, que a primeira vista parece um truque vai se tornar bastante importante na extensão do conceito de atratores para processos. De fato, esta propriedade de atração pullback juntamente com a invariância nos conduzirá ao conceito de atratores pullback para processos.

Note que, em muitas situações, a atração pullback $(s \longrightarrow-\infty)$ e a atração forwards $(t \longrightarrow+\infty)$ não estão relacionadas uma à outra. Não é difícil ver que, no caso do (1.8), a única solução global é $x=0$ e que o conjunto $\mathscr{A}(t)$ é obtido como o limite pullback de conjuntos limitados é $\{0\}$ enquanto que o conjunto $[-1,1]$ atrai subconjuntos limitados de $\mathbb{R}$ quando $t \longrightarrow+\infty$ e nenhum 
conjunto fechado menor que este tem esta propriedade.

Apresentaremos agora as noções necessárias para definir a noção de atrator pullback.

Definição 1.4.3 Seja $\{S(t, s): t \geq s\}$ um processo de evolução. Dado $t \in \mathbb{R}$, diremos que o conjunto $B(t) \subset X$ atrai-pullback subconjuntos limitados de $X$ no instante $t$ sob a ação de $\{S(t, s): t \geq s\}$ se

$$
\lim _{s \rightarrow-\infty} \operatorname{dist}(S(t, s) D, B(t))=0
$$

para cada subconjunto limitado $D$ de $X$. Uma família $\{B(t): t \in \mathbb{R}\}$ atrai-pullback subconjuntos limitados de $X$ sob a ação de $\{S(t, s): t \geq s\}$ se $B(t)$ atrai- pullback subconjuntos limitados de $X$ no instante $t$, para cada $t \in \mathbb{R}$.

Definição 1.4.4 Seja $\{S(t, s): t \geq s\}$ um processo de evolução. Dado $t \in \mathbb{R}$, diremos que o conjunto $B(t) \subset X$ absorve-pullback subconjuntos limitados de $X$ no instante $t$ sob a ação de $\{S(t, s): t \geq s\}$ se

$$
\exists T=T(t, D) \leq t \text { tal que } S(t, s) D \subset B(t), \text { para todo } s \leq T
$$

para cada subconjunto limitado $D$ de $X$. Uma família $\{B(t): t \in \mathbb{R}\}$ absorve-pullback subconjuntos limitados de $X$ sob a ação de $\{S(t, s): t \geq s\}$ se $B(t)$ absorve- pullback subconjuntos limitados de $X$ no instante $t$, para cada $t \in \mathbb{R}$.

Se existe uma família $\{B(t): t \in \mathbb{R}\}$ de conjuntos limitados que (atrai-pullback) absorve-pullback conjuntos limitados diremos que $\{S(t, s): t \geq s\}$ é pullback-limitado dissipativo.

Definição 1.4.5 Seja $\{B(t): t \in \mathbb{R}\}$ uma família de subconjuntos de $X$. Diremos que esta família é invariante pelo processo de evolução $\{S(t, s): t \geq s\}$ se

$$
S(t, s) B(s)=B(t), \text { para todo } t \geq s .
$$

Agora podemos definir o atrator pullback para um processo de evolução. 
Definição 1.4.6 Seja $\{S(t, s): t \geq s\}$ um processo de evolução em um espaço métrico X. Diremos que uma família $\{\mathscr{A}(t): t \in \mathbb{R}\}$ de subconjuntos compactos de $X$ é um atrator-pullback para $\{S(t, s): t \geq s\}$ se é invariante, atrai-pullback subconjuntos limitados de X e é a família de conjuntos fechados que é minimal com a propriedade de atrair pullback subconjuntos limitados; isto é, se outra família $\{C(t): t \in \mathbb{R}\}$ de conjuntos fechados atrai pullback subconjuntos limitados de $X$, então $\mathscr{A}(t) \subset C(t)$, para todo $t \in \mathbb{R}$.

Como no caso autônomo, a noção de $\omega$-limite desempenhara um papel importante na teoria de atratores pullback para processos de evolução.

Definição 1.4.7 Seja $\{S(t, s): t \in \mathbb{R}\}$ um processo de evolução em um espaço métrico $X$ e $B$ um subconjunto de X. O $\omega$-limite pullback de B é definido por

$$
\omega(B, t):=\bigcap_{\sigma \leq t} \overline{\bigcup_{s \leq \sigma} S(t, s) B} .
$$

Para cada subconjunto $B$ de $X$, temos que

$$
\begin{array}{r}
\omega(B, t)=\{y \in X: \\
\text { existem sequências }\left\{s_{k}\right\}_{k \in \mathbb{N}}, s_{k} \leq t, s_{k} \rightarrow-\infty \\
\text { e } \left.\left\{x_{k}\right\}_{k \in \mathbb{N}} \text { em } B \text {, tal que } y=\lim _{k \rightarrow \infty} S\left(t, s_{k}\right) x_{k}\right\} .
\end{array}
$$

Alguns dos resultados apresentados a seguir tem provas semelhantes àquelas dos análogos autônomos.

Lema 1.4.8 Seja $\{S(t, s): t \geq s\}$ um processo de evolução em um espaço métrico $X$. Se $B \subset X$, então $S(t, s) \omega(B, s) \subset \omega(B, t)$ para cada $t \geq s$. Se $t \geq s, B$ é tal que $\omega(B, s)$ é compacto e atrai pullback $B$ no instante $s$, então $S(t, s) \omega(B, s)=\omega(B, t)$. Adicionalmente, se $\omega(B, s)$ atrai pullback B no instante s para cada $s \leq t, B$ é um conjunto conexo e $\bigcup_{s \leq t} \omega(B, s) \subset B$ então $\omega(B, t)$ é conexo.

Lema 1.4.9 Seja $\{S(t, s): t \geq s\}$ um processo de evolução em um espaço métrico $X$. Suponha que $B$ é um subconjunto não vazio de $X$ e que, para cada $t \in \mathbb{R}$, existe $\sigma_{t} \leq t$ tal que $\overline{\bigcup_{s \leq \sigma_{t}} S(t, s) B}$ é compacto. Então, para cada $t \in \mathbb{R}, \omega(B, t)$ é não vazio, compacto, atrai pullback $B$ no instante te $\{\omega(B, t): t \in \mathbb{R}\}$ é invariante. 
O primeiro resultado sobre existência de atratores pullback é uma generalização de um resultado análogo para processos autônomos:

Teorema 1.4.10 Seja $\{S(t, s): t \geq s\}$ um processo de evolução em um espaço métrico X. Então, as seguintes afirmativas são equivalentes

- $\{S(t, s): t \geq s\}$ tem um atrator pullback $\{\mathscr{A}(t): t \in \mathbb{R}\}$.

- Existe uma família de conjuntos compactos $\{K(t): t \in \mathbb{R}\}$ que atrai pullback subconjuntos limitados de $X$ sob a ação de $\{S(t, s): t \geq s\}$.

Em qualquer dos casos

$$
\mathscr{A}=\overline{\bigcup\{\omega(B, t): B \subset X, B \text { limitado }\}} .
$$

O conceito a seguir é útil nas aplicações para obter a existência de atratores pullback sem ter que exibir uma família de conjuntos compactos que atrai pullback subconjuntos limitados.

Definição 1.4.11 Um processo de evolução $\{S(t, s): t \geq s\}$ em um espaço métrico $X$ é dito pullback assintoticamente compacto se, para cada $t \in \mathbb{R}$, sequência $\left\{s_{k}\right\}_{k \in \mathbb{N}}, s_{k} \leq t$ e sequência limitada $\left\{x_{k}\right\}_{k \in \mathbb{N}}$ em $X$ tais que

- $s_{k} \rightarrow-\infty$, quando $k \rightarrow \infty \quad e$

- $\left\{S\left(t, s_{k}\right) x_{k}\right\}_{k \in \mathbb{N}}$ é limitado,

a sequência $\left\{S\left(t, s_{k}\right) x_{k}\right\}_{k \in \mathbb{N}}$ tem uma subsequência convergente.

Lema 1.4.12 $S e\{S(t, s): t \geq s\}$ é um processo de evolução pullback assintoticamente compacto e $B$ é um subconjunto não vazio e limitado de $X$ para o qual, dado $t \in \mathbb{R}$ existe $\sigma_{t} \leq t$ tal que $\bigcup_{\tau \leq \sigma_{t}} S(t, \tau) B$ é limitado, então $\omega(B, t)$ é não vazio, compacto, atrai pullback $B$ no instante te $\{\omega(B, t): t \in \mathbb{R}\}$ é invariante. 
Teorema 1.4.13 Se $\{S(t, s): t \geq s\}$ é pullback limitado dissipativo (veja definição 1.4.4) e pullback assintoticamente compacto (veja definição 1.4.11), então $\mathscr{A}(t)$ dado por

$$
\mathscr{A}(t)=\bigcup\{\omega(B, t): B \subset X, B \text { limitado }\}
$$

é limitado, atrai pullback subconjuntos limitados de $X$ no instante $t$, a família $\{\mathscr{A}(t): t \in \mathbb{R}\}$ é invariante e, se $\{C(t): t \in \mathbb{R}\}$ atrai pullback subconjuntos limitados de $X$, então $A(t) \subset \overline{C(t)}$ para todo $t \in \mathbb{R}$.

Observe que os resultados anteriores não concluem a compacidade da seção $\mathscr{A}(t)$ (nem mesmo se o espaço é de dimensão finita). Isto mostra uma primeira diferença entre os processos de evolução não-autônomos e os autônomos. Para obter a compacidade de cada seção teremos que fazer hipóteses adicionais sobre os processos de evolução.

Definição 1.4.14 Diremos que um processo de evolução $\{S(t, s): t \geq s\}$ é pullback fortemente limitado dissipativo se, para cada $t \in \mathbb{R}$, existir um subconjunto limitado $B(t)$ de $X$ que pullback absorve subconjuntos limitados de X no instante $\tau$ para cada $\tau \leq t$; isto é, dado qualquer subconjunto limitado $D$ de $X$ e $\tau \leq t$, existe $s_{0}(\tau, D)$ tal que $S(\tau, s) D \subset B(t)$, para todo $s \leq s_{0}(\tau, D)$.

O teorema a seguir dá condições suficientes para a existência de um atrator pullback.

Teorema 1.4.15 Se um processo de evolução $\{S(t, s): t \geq s\}$ é pullback fortemente limitado dissipativo e pullback assintoticamente compacto, então $\{S(t, s): t \geq s\}$ tem um atrator pullback $\{\mathscr{A}(t): t \in \mathbb{R}\}$ com a propriedade que $\bigcup_{s \leq t} \mathscr{A}(s)$ é limitado para cada $t \in \mathbb{R}$.

\subsection{Bases de atração e atratores $\mathscr{D}$-pullback}

Considere o conjunto $\mathscr{D}$ composto por famílias de conjuntos de $X$ que satisfazem determinadas propriedades. Estes conjuntos de famílias são chamados bases de atração ou universos. A base de atração mais simples e mais natural é aquele em que as famílias estão sempre formada pelo mesmo conjunto limitado, como seria o caso mais semelhante aos sistemas dinâmicos autônomos. No 
entanto, no caso estocástico é comum encontrar famílias formada por conjuntos limitados, que são diferentes umas das outras. Nesta seção, generalizaremos os resultados. Para definir os conceitos pullback, vamos sempre considerar uma base de atração fixa, mas não determinada.

No que se segue, consideramos a família $\mathscr{M}$ composta por todos as aplicações de $\mathbb{R}$ em $2^{X}$,

$$
D^{\prime}: \mathbb{R} \ni t \mapsto D^{\prime}(t) \in 2^{X}
$$

Se $D^{\prime}$ e $D^{\prime \prime}$ são duas aplicações de $\mathbb{R}$ em $2^{X}$, entenderemos a inclusão, $D^{\prime} \subset D^{\prime \prime}$ quando $D^{\prime}(t) \subset$ $D^{\prime \prime}(t)$ para todo $t \in \mathbb{R}$. Um subconjunto $\mathscr{D}$ de $\mathscr{M}$ é dito fechado por inclusão se, dados $D^{\prime} \in \mathscr{D}$ e $D^{\prime \prime} \in \mathscr{M}$ com $D^{\prime \prime} \subset D^{\prime}$, se cumpre que $D^{\prime \prime} \in \mathscr{D}$.

Definição 1.5.1 Um subconjunto $\mathscr{D} \subset \mathscr{M}$, fechado por inclusão e tal que as aplicações $D^{\prime}$ em $\mathscr{D}$ satisfazem que $D^{\prime}(t) \neq \emptyset$ para todo $t \in \mathbb{R}$, é chamado base de atração ou universo.

O exemplo mais simples deste tipo de famílias de aplicações é dada por $D(t) \equiv D$ para todo $t \in \mathbb{R}$ onde $D$ é um subconjunto limitado de $\mathrm{X}$.

Outro exemplo muito comum, no caso de sistemas dinâmicos aleatorios é fornecido pelos conjuntos $\mathscr{D}$ composto por conjuntos que possuem crescimento sub-exponencial em relação ao tempo, isto é

$$
\sup _{x \in D^{\prime}(t)}\|x\| \leq g(t) e^{-t}
$$

para alguma função positiva $g=g(s)$, tal que, $g(s) e^{-s} \rightarrow 0$ quando $|s| \rightarrow \infty$.

Definição 1.5.2 Seja $\mathscr{D}$ uma base de atração. Dado $t \in \mathbb{R}, B(t) \subset X$ é dito pullback $\mathscr{D}$-absorvente no instante $t$ se, para cada $D \in \mathscr{D}$ existe $s_{0}=s_{0}(t, D) \leq t$ tal que

$$
S(t, s) D(s) \subset B(t), \text { para todo } s \leq s_{0}
$$

A família $\{B(t): t \in \mathbb{R}\}$ é chamado pullback $\mathscr{D}$-absorvente se, $B(t)$ é pullback $\mathscr{D}$-absorvente no instante $t$, para todo $t \in \mathbb{R}$. 
A definição anterior é uma generalização da definição 1.4.4.

Definição 1.5.3 Uma família de conjuntos compactos $\left\{\mathscr{A}_{\mathscr{D}}: t \in \mathbb{R}\right\}$ é chamado $\mathscr{D}$-atrator pullback associado ao processo $\{S(t, s): t \geq s\}$ se:

1. A família $\left\{\mathscr{A}_{\mathscr{D}}: t \in \mathbb{R}\right\}$ é invariante, isto é,

$$
S(t, s) \mathscr{A}_{\mathscr{D}}(s)=\mathscr{A}_{\mathscr{D}}(t) \text { para todo } t \geq s,
$$

2. A família $\left\{\mathscr{A}_{\mathscr{D}}: t \in \mathbb{R}\right\}$ pullback $\mathscr{D}$-atrai todo elemento de $\mathscr{D}$, isto é,

$$
\lim _{s \rightarrow-\infty} \operatorname{dist}\left(S(t, s) D(s), \mathscr{A}_{\mathscr{D}}(t)\right)=0, \text { para todo } t \in \mathbb{R}
$$

Exemplos 1.5.4 Diferentes universos vai dar origem a diferentes atratores, refletindo diferentes aspectos da dinâmica. De fato, considere o exemplo

$$
x^{\prime}=f(t, x) \quad x(\tau)=x_{0} \in \mathbb{R}
$$

onde $f: \mathbb{R}^{2} \longrightarrow \mathbb{R}$ é a função

$$
f(t, x)=\left\{\begin{array}{cl}
-x, & x \in\left[-e^{-t}, e^{-t}\right] \\
-x-x\left(x-e^{-t}\right) e^{t}, & e^{-t} \leq|x| \leq 2 e^{-t} \\
-2 x, & |x| \geq 2 e^{-t}
\end{array}\right.
$$

Se $\mathscr{D}$ contém $D(t)=\left\{\left[-e^{-t}, e^{-t}\right]: t \in \mathbb{R}\right\}$, então o atrator no sentido $\mathscr{D}$-pullback $\mathscr{A}_{\mathscr{D}}$ satisfará

$$
\left[-e^{-t}, e^{-t}\right] \subset \mathscr{A}_{\mathscr{D}}(t) \subset\left[-2 e^{-t}, 2 e^{-t}\right]
$$

Por outro lado, se apenas deseja atrair conjuntos limitados, então o atrator pullback será $\mathscr{A}($.$) ,$ com $\mathscr{A}(t)=\{0\}$, para todo $t \in \mathbb{R}$.

Dentro deste contexto também é natural tentar encontrar o maior universo possível $\mathscr{D}$ para os 
quais existem um atrator no sentido $\mathscr{D}$-pullback. Se consideramos à equação escalar simples

$$
x^{\prime}=-\alpha x+f(t)
$$

recordamos que a solução explícita é

$$
x(t)=e^{-\alpha(t-s)} x(s)+\int_{s}^{t} e^{-\alpha(t-r)} f(r) d r
$$

Notemos que se

$$
\int_{-\infty}^{0} e^{\alpha r} f(r) d r \text { converge }
$$

então

$$
x^{*}(t)=\int_{-\infty}^{t} e^{-\alpha(t-r)} f(r) d r
$$

atrai no sentido pullback subconjuntos limitados de condições iniciais. Mas é evidente que se pode, de fato, deixar $x(s)$ crescer como $s \rightarrow-\infty$, desde que $e^{\alpha s} x(s) \rightarrow 0$ quando $s \rightarrow-\infty$, assim pode-se tomar

$$
\left\{x(.): e^{\alpha s} x(s) \rightarrow 0 \text { quando } s \rightarrow-\infty\right\}
$$

como o universo $\mathscr{D}$.

Antes de dar um resultado análogo ao Teorema 1.4.15, precisamos definir os conceitos de $\omega$ limite e processo pullback assintoticamente compacto neste caso.

Definição 1.5.5 O conjunto pullback $\omega$-limite de uma família $D \in \mathscr{D}$ é definido como

$$
\omega(D, t):=\bigcap_{\sigma \leq t} \overline{\bigcup_{s \leq \sigma} S(t, s) D(s)} .
$$

Definição 1.5.6 O processo $\{S(t, s): t \geq s\}$ é chamado pullback $\mathscr{D}$-assintoticamente compacto se para cada $t \in \mathbb{R}$, sequências $\left\{s_{n}\right\}_{n \in \mathbb{N}}$ com $s_{n} \rightarrow-\infty$ quando $n \rightarrow \infty e\left\{x_{n}\right\}_{n \in \mathbb{N}}$ com $x_{n} \in D\left(s_{n}\right)$ para algum $D \in \mathscr{D}$, tem-se que $\left\{S\left(t, s_{n}\right) x_{n}\right\}_{n \in \mathbb{N}}$ tem uma subsequência convergente. 
Agora podemos estabelecer um resultado garantindo a existência de $\mathscr{D}$-atratores pullback.

Teorema 1.5.7 Suponhamos que o processo $\{S(t, s): t \geq s\}$ seja pullback $\mathscr{D}$-assintoticamente compacto, e que $\widehat{B}=\{B(t): t \in \mathbb{R}\}$ seja uma família pullback $\mathscr{D}$-absorvente para $\{S(t, s): t \geq s\}$.

Então, a família

$$
\left\{\mathscr{A}_{\mathscr{D}}(t): t \in \mathbb{R}\right\}=\{\omega(\widehat{B}, t): t \in \mathbb{R}\}
$$

é um $\mathscr{D}$-atrator pullback para $\{S(t, s): t \geq s\}$.

Além disso, $\left\{\mathscr{A}_{\mathscr{D}}(t): t \in \mathbb{R}\right\}$ é minimal no sentido de que se $\widehat{C}=\{C(t): t \in \mathbb{R}\}$ é uma família de subconjuntos fechados não vazios, tal que

$$
\lim _{s \rightarrow-\infty} \operatorname{dist}(S(t, s) B(s), C(t))=0
$$

para qualquer $t \in \mathbb{R}$. Então $\mathscr{A}_{\mathscr{D}}(t) \subset C(t)$ para todo $t \in \mathbb{R}$.

A seguinte pergunta natural é a relação entre o atrator pullback $\{\mathscr{A}(t): t \in \mathbb{R}\}$ definido na seção 1.2 e o $\mathscr{D}$-atrator pullback $\left\{\mathscr{A}_{\mathscr{D}}: t \in \mathbb{R}\right\}$ definido nesta seção. Se supusermos que a base da atração $\mathscr{D}$ inclui todos os subconjuntos limitados de $X$, temos que, graças a minimalidade do atrator pullback, $\mathscr{A}(t) \subset \mathscr{A}_{\mathscr{D}}(t)$ para todo $t \in \mathbb{R}$. Marín-Rubio e Real dão em [71] o seguinte resultado de igualdade.

Teorema 1.5.8 Seja $\{S(t, s): t \geq s\}$ um processo que é pullback $\mathscr{D}$-assintoticamente compacto e que existe uma família $\widehat{B}=\{B(t): t \in \mathbb{R}\}$ de subconjuntos limitados que é pullback $\mathscr{D}$-absorvente para cada $\tau \leq t$. Se $\mathscr{D}$ contém os subconjuntos limitados de $X$, então o $\mathscr{D}$-atrator pullback $\left\{\mathscr{A}_{\mathscr{D}}(t)\right.$ : $t \in \mathbb{R}\}$ coincide com o atrator pullback $\{\mathscr{A}(t): t \in \mathbb{R}\}$ de subconjuntos limitados de $X$. 


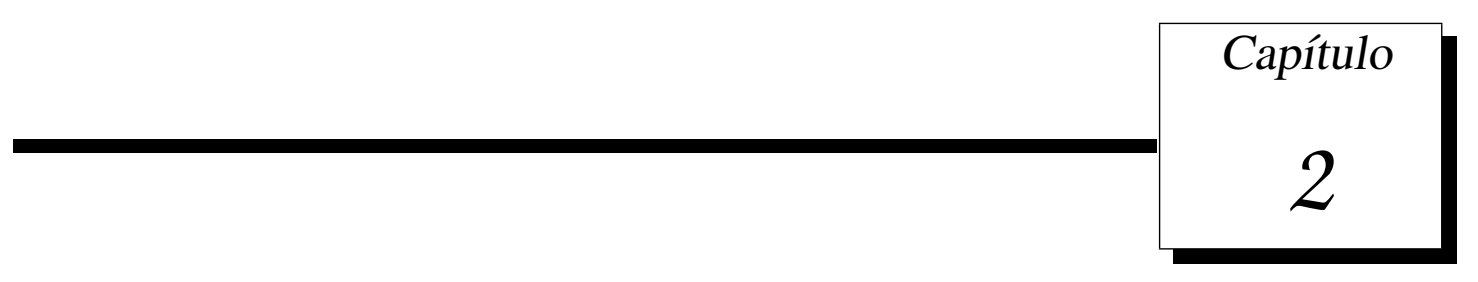

\section{Existência e unicidade de solução fraca para o} problema de onda semilinear

Seja $\mathscr{O}$ um subconjunto aberto e limitado do $\mathbb{R}^{N}$ contendo a origem, com fronteira $\partial \mathscr{O}$ suave.

Seja $\mathscr{O}_{t}=\left\{x=h(t) y: \quad y \in \mathscr{O} ; \quad x_{i}=h(t) y_{i}\right.$ para $\left.i=1,2, \ldots, N.\right\}$. Consideremos em $\widehat{\mathscr{Q}}_{\tau} \mathrm{o}$ seguinte sistema

$$
\left\{\begin{array}{l}
\frac{\partial^{2} u}{\partial t^{2}}+\eta \frac{\partial u}{\partial t}-\Delta u+g(u)=f(x, t) \text { em } \widehat{\mathscr{Q}}_{\tau}, \\
u=0 \text { sobre } \widehat{\Sigma}_{\tau}, \\
u(x, \tau)=u_{\tau 0}(x), \quad \frac{\partial u}{\partial t}(x, \tau)=u_{\tau 1}(x), \quad x \in \mathscr{O}_{\tau}
\end{array}\right.
$$

onde $\eta>0$, e

$$
\widehat{\mathscr{Q}}_{\tau}=\bigcup_{t \in(\tau,+\infty)} \mathscr{O}_{t} \times\{t\} \text { e } \widehat{\Sigma}_{\tau}=\bigcup_{t \in(\tau,+\infty)} \partial \mathscr{O}_{t} \times\{t\}
$$

Para obtermos os resultados de existência e unicidade de soluções do sistema (2.1) considere- 
mos para cada $T>\tau$, o problema auxiliar

$$
\left\{\begin{array}{l}
\frac{\partial^{2} u}{\partial t^{2}}+\eta \frac{\partial u}{\partial t}-\Delta u+g(u)=f(x, t) \text { em } \widehat{\mathscr{Q}}_{\tau, T}, \\
u=0 \text { sobre } \widehat{\Sigma}_{\tau, T}, \\
u(x, \tau)=u_{\tau 0}(x), \quad \frac{\partial u}{\partial t}(x, \tau)=u_{\tau 1}(x), x \in \mathscr{O}_{\tau}
\end{array}\right.
$$

onde

$$
\widehat{\mathscr{Q}}_{\tau, T}=\bigcup_{t \in(\tau, T)} \mathscr{O}_{t} \times\{t\} \text { e } \widehat{\Sigma}_{\tau, T}=\bigcup_{t \in(\tau, T)} \partial \mathscr{O}_{t} \times\{t\}
$$

Neste trabalho usaremos $(\cdot, \cdot)_{t},|\cdot, \cdot|_{t} \mathrm{e}((\cdot, \cdot))_{t},\|\cdot, \cdot\|_{t}$ para denotar o produto interno e a norma nos espaços $L^{2}\left(\mathscr{O}_{t}\right)$ e $H_{0}^{1}\left(\mathscr{O}_{t}\right)$, respectivamente.

\subsection{Existência e unicidade de solução forte para o sistema auxiliar $(2.2)$}

Para estudar a existência de soluções fortes do sistema (2.2) vamos supor as seguintes hipóteses:

(H.1) $h \in C^{2}(\mathbb{R}), \quad$ com $0<h_{0} \leq h(t) \leq h_{1}$ e $h^{\prime}(t) \geq 0$ para todo $t \in \mathbb{R}$.

(H.2) Para cada $T \geq \tau, \quad \gamma D<1$, onde $\gamma=\gamma_{\tau, T}=\max _{[\tau, T]}\left|h^{\prime}(t)\right|$ e $\quad D \quad$ é o diametro de $\mathscr{O}$.

(H.3) $g \in C^{1}(\mathbb{R}), \quad\left|g^{\prime}(s)\right| \leq C\left(1+|s|^{\frac{2}{N-2}}\right)$ e $g(s) s \geq 0, \quad$ para todo $s \in \mathbb{R}$.

Na hipótese (H.1) a condição $h^{\prime}(t) \geq 0$ significa que o domínios $\Omega_{t}$ são crescentes com respeito a $t$. A condição (H.2) implica que a fronteira lateral $\Sigma$ é time-like. Em outras palavras, se $v=$ $\left(v_{x}, v_{t}\right)$ denota o vetor normal unitário à $\widehat{\Sigma}$, então

$$
\left|v_{t}\right|<\left|v_{x}\right| .
$$


Faremos uma mudança de variáveis para transformar o dominio $\widehat{\mathscr{Q}}_{\tau, T}$ em um domínio cilíndrico $\mathscr{Q}_{\tau, T}=\mathscr{O} \times(\tau, T)$. Observe que quando $(x, t)$ varia em $\widehat{\mathscr{Q}}_{\tau, T}$, o ponto $(y, t)$ de $\mathbb{R}^{N}$, com $y=h(t)^{-1} x$ varia no cilíndro $\mathscr{Q}_{\tau, T}$. Assim, definimos a aplicação

$$
r: \widehat{\mathscr{Q}}_{\tau, T} \longrightarrow \mathscr{Q}_{\tau, T}
$$

dado por $r(x, t)=(y, t)$ onde $y=h(t)^{-1} x$. Note que aplicação $r$ é um difeomorfismo de classe $C^{2}$.

Fazendo a mudança de variável $v(y, t)=u(h(t) y, t)$ e aplicando no problema (2.2), obtemos o seguinte sistema equivalente

$$
\left\{\begin{array}{l}
\frac{\partial^{2} v}{\partial t^{2}}+\eta \frac{\partial v}{\partial t}+A(t) v+a_{2}(y, t) \cdot \nabla \frac{\partial v}{\partial t}+a_{3}(y, t) \cdot \nabla v+g(v)=f(h(t) y, t)=\bar{f}(y, t) \text { em } \mathscr{Q}_{\tau, T}, \\
v=0 \text { em } \Sigma_{\tau, T}, \\
v(y, \tau)=v_{\tau 0}(y), \quad \frac{\partial v}{\partial t}(y, \tau)=v_{\tau 1}(y), y \in \mathscr{O},
\end{array}\right.
$$

onde

$$
\begin{aligned}
& A(t) v=-\sum_{k, l=1}^{N} \frac{\partial}{\partial y_{l}}\left(a_{k l}(y, t) \frac{\partial v}{\partial y_{k}}\right), \\
& a_{k l}=\sum_{j=1}^{N} \beta_{k j} \beta_{l j}-\left[\frac{h^{\prime}(t)}{h(t)}\right]^{2} y_{k} y_{l}, \beta_{k l}=\frac{1}{h(t)} \text { quando } k=l \text { e } \beta_{k l}=0 \text { quando } k \neq l, \\
& a_{2}(y, t)=-2 \frac{h^{\prime}(t)}{h(t)} y, \\
& a_{3}(y, t)=-\frac{1}{h(t)^{2}}\left[h^{\prime \prime}(t) h(t)+(N-1)\left(h^{\prime}(t)+\eta h(t)\right) h^{\prime}(t)\right] y .
\end{aligned}
$$

Sejam $(()),,\|$,$\| e (),,|$,$| o produto escalar e a norma em H_{0}^{1}(\mathscr{O})$ e $L^{2}(\mathscr{O})$, respectivamente. Denotemos por $a(t, v, w)$ a forma bilinear, contínua e simétrica, definida em $H_{0}^{1}(\mathscr{O})$ por

$$
a(t, v, w)=\sum_{k, l=1}^{N} \int_{\mathscr{O}} a_{k l}(y, t) \frac{\partial v}{\partial y_{k}} \frac{\partial w}{\partial y_{l}} d y
$$


Da hipótese (H.2) temos que existe $h_{\tau, T}$ dependendo de $\tau$ e $T$ tal que

$$
a(t, v, v) \geq h_{\tau, T}\|v\|^{2}
$$

portanto, $a(t, v, w)$ é coercivo.

\subsubsection{Existência de solução forte para o sistema (2.3)}

A existência de solução para o problema (2.3) será estabelecida utilizando o Método de FaedoGalerkin, o qual consiste em projetar o nosso problema em espaços de dimensão finita, de modo a obter um problema aproximado em um espaço de dimensão finita e depois, por meio de estimativas à priori, em uma topologia adequada fazer a passagem ao limite das soluções aproximadas e o limite dessas soluções é o que nos dá a solução do problema (2.3).

Teorema 2.1.1 Seja $\bar{f} \in H_{l o c}^{1}\left(\mathbb{R}, L^{2}(\mathscr{O})\right)$, sobre as hipóteses (H.1), (H.2), (H.3). Então, se $v_{\tau 0} \in$ $H_{0}^{1}(\mathscr{O}) \cap H^{2}(\mathscr{O})$ e $v_{\tau 1} \in H_{0}^{1}(\mathscr{O})$, existe uma função $v: \mathscr{Q}_{\tau, T} \longrightarrow \mathbb{R}$ que é solução forte do problema (2.3), isto é,

$$
v \in L^{\infty}\left(\tau, T ; H_{0}^{1}(\mathscr{O}) \cap H^{2}(\mathscr{O})\right) ; \quad \frac{\partial v}{\partial t} \in L^{\infty}\left(\tau, T ; H_{0}^{1}(\mathscr{O})\right) e \frac{\partial^{2} v}{\partial t^{2}} \in L^{\infty}\left(\tau, T ; L^{2}(\mathscr{O})\right)
$$

Demonstração: Consideremos uma base Hilbertiana $\left(w_{k}\right)_{k \in \mathbb{N}}$ de $H_{0}^{1}(\mathscr{O})$, constituída pelas funções próprias do operador $(-\Delta)$ e seja $V_{m}=\left[w_{1}, w_{2}, \ldots, w_{m}\right]$ o subespaço de $H_{0}^{1}(\mathscr{O})$ gerado pelos $m$ primeiros vetores de $\left(w_{k}\right)_{k \in \mathbb{N}}$. Ainda para cada $m \in \mathbb{N}$, procuramos encontrar uma função $v_{m} \in V_{m}$, tal que

$$
v_{m}(y, t)=\sum_{k=1}^{m} d_{k m}(t) w_{k}(y)
$$


onde $v_{m} \in V_{m}$ é solução do problema variacional associado ao sistema (2.3), dado por

$$
\left\{\begin{array}{l}
\left(v_{m}^{\prime \prime}, w_{k}\right)+\eta\left(v_{m}^{\prime}, w_{k}\right)+a\left(t, v_{m}, w_{k}\right)+\left(a_{2}(y, t) \cdot \nabla v_{m}^{\prime}, w_{k}\right)+\left(a_{3}(y, t) \cdot \nabla v_{m}, w_{k}\right) \\
+\left(g\left(v_{m}\right), w_{k}\right)=\left(\bar{f}(y, t), w_{k}\right), \quad 1 \leq k \leq m, \tau \leq t \leq T \\
v_{m}(\tau)=v_{0 \tau m}=\Sigma_{k=1}^{m}\left(v_{\tau 0}, w_{k}\right) w_{k} \rightarrow v_{\tau 0} \text { em } H_{0}^{1}(\mathscr{O}) \cap H^{2}(\mathscr{O}) \\
v_{m}^{\prime}(\tau)=v_{1 \tau m}=\Sigma_{k=1}^{m}\left(v_{\tau 1}, w_{k}\right) w_{k} \rightarrow v_{\tau 1} \text { em } H_{0}^{1}(\mathscr{O})
\end{array}\right.
$$

Notando que $g \in C^{1}(\mathbb{R})$, pelo Teorema de Carathéodory, o sistema (2.5) tem solução local no intervalo $\left(\tau, T_{m}\right)$, com $\tau<T_{m} \leq T$. Podemos estender esta solução ao intervalo $(\tau, T)$ independente de $m$, desde que possamos obter algumas esttimativas à priori.

Estimativa I: Multiplicando a equação $(2.5)_{1}$ por $d_{k m}^{\prime}(t)$, somando de $k=1$ até $m$, tem-se

$$
\begin{aligned}
& \left(v_{m}^{\prime \prime}, v_{m}^{\prime}\right)+\eta\left(v_{m}^{\prime}, v_{m}^{\prime}\right)+a\left(t, v_{m}, v_{m}^{\prime}\right)+\left(a_{2}(y, t) \cdot \nabla v_{m}^{\prime}, v_{m}^{\prime}\right) \\
& +\left(a_{3}(y, t) \cdot \nabla v_{m}, v_{m}^{\prime}\right)+\left(g\left(v_{m}\right), v_{m}^{\prime}\right)=\left(\bar{f}(y, t), v_{m}^{\prime}\right) .
\end{aligned}
$$

Então, obtemos

$$
\begin{aligned}
& \frac{1}{2} \frac{d}{d t}\left|v_{m}^{\prime}\right|^{2}+\eta\left|v_{m}^{\prime}\right|^{2}+a\left(t, v_{m}, v_{m}^{\prime}\right)+\left(a_{2}(y, t) \cdot \nabla v_{m}^{\prime}, v_{m}^{\prime}\right) \\
& +\left(a_{3}(y, t) \cdot \nabla v_{m}, v_{m}^{\prime}\right)+\left(g\left(v_{m}\right), v_{m}^{\prime}\right)=\left(\bar{f}(y, t), v_{m}^{\prime}\right) .
\end{aligned}
$$

Estimando (2.7) etapa por etapa, temos

- $a\left(t, v_{m}, v_{m}^{\prime}\right)=\frac{1}{2} \frac{d}{d t} a\left(t, v_{m}, v_{m}\right)-\frac{1}{2} a^{\prime}\left(t, v_{m}, v_{m}\right)$, onde $a^{\prime}(t, v, w)=\sum_{k, l=1}^{N} \int_{\mathscr{O}} a_{k l}^{\prime}(y, t) \frac{\partial v}{\partial y_{k}} \frac{\partial w}{\partial y_{l}} d y$,

- $\left(a_{2}(y, t) \cdot \nabla v_{m}^{\prime}, v_{m}^{\prime}\right)=-N \frac{h^{\prime}(t)}{h(t)}\left|v_{m}^{\prime}\right|^{2}$,

- $\left(a_{3}(y, t) \frac{\partial v_{m}}{\partial y}, v_{m}^{\prime}\right) \leq C_{1}\left(\|\left. v_{m}\right|^{2}+\left|v_{m}^{\prime}\right|^{2}\right)$,

- $\left(g\left(v_{m}\right), v_{m}^{\prime}\right)=\frac{d}{d t} \int_{\mathscr{O}} G\left(v_{m}\right) d y$,

- $\left(\bar{f}(y, t), v_{m}^{\prime}\right) \leq C_{3}+\frac{1}{2}\left|v_{m}^{\prime}\right|^{2}$. 
Substituindo as equações anteriores em (2.7), obtemos

$$
\frac{d}{d t}\left(\left|v_{m}^{\prime}\right|^{2}+a\left(t, v_{m}, v_{m}\right)+2 \int_{\mathscr{O}} G\left(v_{m}\right) d y\right)+\eta\left|v_{m}^{\prime}\right|^{2} \leq C_{4}\left(\left|v_{m}^{\prime}\right|^{2}+|| v_{m}||^{2}\right)
$$

Integrando (2.8) de $\tau$ até $t$, obtemos

$$
\begin{aligned}
\left|v_{m}^{\prime}\right|^{2}+a\left(t, v_{m}, v_{m}\right)+2 \int_{\mathscr{O}} G\left(v_{m}\right) d y+\eta \int_{\tau}^{t}\left|v_{m}^{\prime}\right|^{2} d t & \leq\left|v_{1 \tau m}\right|^{2}+a_{1}\left(\tau, v_{0 \tau m}, v_{0 \tau m}\right)+2 \int_{\mathscr{O}} G\left(v_{0 \tau m}\right) d y \\
& +\int_{\tau}^{t} C_{4}\left(\left|v_{m}^{\prime}\right|^{2}+\|\left. v_{m}\right|^{2}\right) d t .
\end{aligned}
$$

Desejamos utilizar o lema de Gronwall, para tanto devemos mostrar que

$$
\int_{\mathscr{O}} G\left(v_{0} \tau m\right) d y \leq C
$$

onde $C$ é uma constante independente de $m$.

De fato, como $s g(s) \geq 0$ e $0 \leq s \leq t$, segue que $g(s) \geq 0$. Da continuidade de $g$, obtemos

$$
G(t)=\int_{0}^{t} g(s) \geq 0, \text { para todo } t \in(0, T)
$$

Além disso, devemos notar que $g(0)=0$. Com isso, obtemos

$$
\int_{\mathscr{O}} G\left(v_{0 \tau m}\right) d y \leq \int_{\mathscr{O}}\left|G\left(v_{0 \tau \tau}\right) d y\right| \leq \int_{\mathscr{O}} \int_{0}^{v_{0 \tau m}}|g(s)| d s d y \leq \int_{\mathscr{O}} \int_{0}^{v_{0 \tau m}} C\left(1+|s|^{\frac{N}{N-2}}\right) d s d y .
$$

Usando o fato que a imersão $H_{0}^{1}(\mathscr{O}) \hookrightarrow L^{\frac{2 N-2}{N-2}}(\mathscr{O})$ é contínua, decorre de $(2.5)_{3}$ que

$$
\int_{\mathscr{O}} G\left(v_{0 \tau m}\right) d y \leq C
$$

Da coercitividade de $a(t, v, w)$ e Gronwall, tem-se

$$
\left|v_{m}^{\prime}\right|^{2}+\| v_{m}||^{2}+\eta \int_{\tau}^{t}\left|v_{m}^{\prime}\right|^{2} d t \leq C_{5} e^{C_{4}(T-\tau)}
$$


De (2.9), utilizando o Teorema de Prolongamento, estendemos a solução ao intervalo $(\tau, T)$.

\section{Estimativa II:}

Nosso objetivo agora é obter uma estimativa para $v_{m}^{\prime \prime}(t)$ e para tanto consideramos a derivada em relação à $t$ em $(2.5)_{1}$, isto é,

$$
\begin{aligned}
& \left(v_{m}^{\prime \prime \prime}, w_{k}\right)+\eta\left(v_{m}^{\prime \prime}, w_{k}\right)+a\left(t, v_{m}^{\prime}, w_{k}\right)+a^{\prime}\left(t, v_{m}, w_{k}\right)+\left(a_{2}(y, t) \cdot \nabla v_{m}^{\prime}, w_{k}\right) \\
& \quad+\left(a_{2}^{\prime}(y, t) \cdot \nabla v_{m}^{\prime}, w_{k}\right)+\left(a_{3}(y, t) \cdot \nabla v_{m}^{\prime}, w_{k}\right)+\left(a_{3}^{\prime}(y, t) \cdot \nabla v_{m}, w_{k}\right)+\left(g^{\prime}\left(v_{m}\right) v_{m}^{\prime}, w_{k}\right) \\
& =\left(\frac{\partial f}{\partial y}(h(t) y, t) h^{\prime}(t) y+f^{\prime}(h(t) y, t), w_{k}\right) .
\end{aligned}
$$

Considerando $w_{k}=v_{m}^{\prime \prime}$, obtemos

$$
\begin{aligned}
& \left(v_{m}^{\prime \prime \prime}, v_{m}^{\prime \prime}\right)+\eta\left(v_{m}^{\prime \prime}, v_{m}^{\prime \prime}\right)+a\left(t, v_{m}^{\prime}, v_{m}^{\prime \prime}\right)+a^{\prime}\left(t, v_{m}, v_{m}^{\prime \prime}\right)+\left(a_{2}(y, t) \cdot \nabla v_{m}^{\prime \prime}, v_{m}^{\prime \prime}\right) \\
& \quad+\left(a_{2}^{\prime}(y, t) \cdot \nabla v_{m}^{\prime}, v_{m}^{\prime \prime}\right)+\left(a_{3}(y, t) \cdot \nabla v_{m}^{\prime}, v_{m}^{\prime \prime}\right)+\left(a_{3}^{\prime}(y, t) \cdot \nabla v_{m}, v_{m}^{\prime \prime}\right)+\left(g^{\prime}\left(v_{m}\right) v_{m}^{\prime}, v_{m}^{\prime \prime}\right) \\
& =\left(\frac{\partial f}{\partial y}(h(t) y, t) h^{\prime}(t) y+f^{\prime}(h(t) y, t), v_{m}^{\prime \prime}\right) .
\end{aligned}
$$

Analizando os termos em (2.11), temos

$$
\begin{aligned}
& \left(v_{m}^{\prime \prime \prime}, v_{m}^{\prime \prime}\right)=\frac{1}{2} \frac{d}{d t}\left|v_{m}^{\prime \prime}\right|^{2} \\
& \eta\left(v_{m}^{\prime \prime}, v_{m}^{\prime \prime}\right)=\eta\left|v_{m}^{\prime \prime}\right|^{2} \\
& a\left(t, v_{m}^{\prime}, v_{m}^{\prime \prime}\right)=\frac{1}{2} \frac{d}{d t} a\left(t, v_{m}^{\prime}, v_{m}^{\prime}\right)-\frac{1}{2} a^{\prime}\left(t, v_{m}^{\prime}, v_{m}^{\prime}\right) \\
& a^{\prime}\left(t, v_{m}, v_{m}^{\prime \prime}\right)=\frac{d}{d t} a^{\prime}\left(t, v_{m}, v_{m}^{\prime}\right)-a^{\prime \prime}\left(t, v_{m}, v_{m}^{\prime}\right)-a^{\prime \prime}\left(t, v_{m}, v_{m}\right)-a^{\prime}\left(t, v_{m}^{\prime}, v_{m}^{\prime}\right)
\end{aligned}
$$


Substituindo as equações anteriores em (2.11), obtemos

$$
\begin{aligned}
\frac{1}{2} \frac{d}{d t} & {\left[\left|v_{m}^{\prime \prime}\right|^{2}+a\left(t, v_{m}^{\prime}, v_{m}^{\prime}\right)+2 a^{\prime}\left(t, v_{m}, v_{m}^{\prime}\right)\right]+\eta\left|v_{m}^{\prime \prime}\right|^{2} } \\
= & \frac{3}{2} a^{\prime}\left(t, v_{m}^{\prime}, v_{m}^{\prime}\right)+a^{\prime \prime}\left(t, v_{m}, v_{m}^{\prime}\right)-\left(a_{2}(y, t) \cdot \nabla v_{m}^{\prime \prime}, v_{m}^{\prime \prime}\right) \\
& -\left(a_{2}^{\prime}(y, t) \cdot \nabla v_{m}^{\prime}, v_{m}^{\prime \prime}\right)-\left(a_{3}(y, t) \cdot \nabla v_{m}^{\prime}, v_{m}^{\prime \prime}\right)-\left(a_{3}^{\prime}(y, t) \cdot \nabla v_{m}, v_{m}^{\prime \prime}\right) \\
& -\left(g^{\prime}\left(v_{m}\right) v_{m}^{\prime}, v_{m}^{\prime \prime}\right)+\left(\frac{\partial f}{\partial y}(h(t) y, t) h^{\prime} y+f^{\prime}(h(t) y, t), v_{m}^{\prime \prime}\right) .
\end{aligned}
$$

Estimando os termos do lado direito da equação (2.12), temos

$$
\begin{aligned}
& \frac{3}{2} a^{\prime}\left(t, v_{m}^{\prime}, v_{m}^{\prime}\right) \leq C_{6}\left\|v_{m}^{\prime}\right\|^{2}, \\
& \left.-\left(a_{3}(y, t)\right) \cdot \nabla v_{m}^{\prime}, v_{m}^{\prime \prime}\right) \leq C_{7}\left(|| v_{m}^{\prime} \|^{2}+\left|v_{m}^{\prime \prime}\right|^{2}\right), \\
& \left.-\left(a_{2}^{\prime}(y, t)\right) \cdot \nabla v_{m}^{\prime}, v_{m}^{\prime \prime}\right) \leq C_{7}^{\prime}\left(\left.|| v_{m}^{\prime}\right|^{2}+\left|v_{m}^{\prime \prime}\right|^{2}\right), \\
& -\left(a_{3}^{\prime}(y, t) \cdot \nabla v_{m}, v_{m}^{\prime \prime}\right) \leq C_{8}\left(\left.|| v_{m}\right|^{2}+\left|v_{m}^{\prime \prime}\right|^{2}\right) \\
& \left.-\left(a_{2}(y, t)\right) \cdot \nabla v_{m}^{\prime \prime}, v_{m}^{\prime \prime}\right) \leq C_{9}\left|v_{m}^{\prime \prime}\right|^{2}, \\
& \left|-\left(g^{\prime}\left(v_{m}\right) v_{m}^{\prime}, v_{m}^{\prime \prime}\right)\right| \leq C \int_{\mathscr{O}}\left(1+\left|v_{m}\right| \frac{2}{N-2}\right)\left|v_{m}^{\prime}\right|\left|v_{m}^{\prime \prime}\right| d y \leq C_{12}\left|v_{m}^{\prime \prime}\right|^{2}, \\
& \left(\frac{\partial f}{\partial y}(h(t) y, t) h^{\prime} y+f^{\prime}(h(t) y, t), v_{m}^{\prime \prime}\right) \leq C_{13}\left|v_{m}^{\prime \prime}\right|^{2} .
\end{aligned}
$$

Substituindo as estimativas anteriores em (2.12), resulta

$$
\frac{1}{2} \frac{d}{d t}\left[\left|v_{m}^{\prime \prime}\right|^{2}+a\left(t, v_{m}^{\prime}, v_{m}^{\prime}\right)+2 a^{\prime}\left(t, v_{m}, v_{m}^{\prime}\right)\right]+\eta\left|v_{m}^{\prime \prime}\right|^{2} \leq C_{14}\left(|| v_{m}^{\prime} \|^{2}+\left|v_{m}^{\prime \prime}\right|^{2}\right)
$$

Integrando (2.13) de $\tau$ até $t$, obtemos

$$
\begin{aligned}
\left|v_{m}^{\prime \prime}\right|^{2} & +a\left(t, v_{m}^{\prime}, v_{m}^{\prime}\right)+2 a^{\prime}\left(t, v_{m}, v_{m}^{\prime}\right)+\eta \int_{\tau}^{t}\left|v_{m}^{\prime \prime}\right|^{2} d t \\
\leq & \left|v_{m}^{\prime \prime}(\tau)\right|^{2}+a\left(\tau, v_{1 \tau m}, v_{1 \tau m}\right)+2 a^{\prime}\left(\tau, v_{0 \tau m}, v_{1 \tau m}\right)+ \\
& +C_{14} \int_{\tau}^{t}\left(\|\left. v_{m}^{\prime}\right|^{2}+\left|v_{m}^{\prime \prime}\right|^{2}\right) .
\end{aligned}
$$

Segue, do sistema (2.5), que $\left|v_{m}^{\prime \prime}(\tau)\right|^{2}$ é limitado.

Note que $\left|a^{\prime}\left(t, v_{m}, v_{m}^{\prime}\right)\right| \leq C_{15}|| v_{m} \|^{2}+\frac{1}{4} a\left(t, v_{m}^{\prime}, v_{m}^{\prime}\right)$, logo substituindo em (2.14) e utilizando o 
Lema de Gronwall, temos

$$
\left|v_{m}^{\prime \prime}\right|^{2}+|| v_{m}^{\prime} \|^{2}+\eta \int_{\tau}^{t}\left|v_{m}^{\prime \prime}\right|^{2} d t \leq C_{16} e^{C_{14}(T-\tau)} .
$$

\section{Estimativa III:}

Considerando $w_{k}=-\Delta v_{m}$ em $(2.5)_{1}$, obtemos

$$
\begin{gathered}
\left(v_{m}^{\prime \prime},-\Delta v_{m}\right)+\eta\left(v_{m}^{\prime},-\Delta v_{m}\right)+a\left(t, v_{m},-\Delta v_{m}\right)+\left(a_{2}(y, t) \cdot \nabla v_{m}^{\prime},-\Delta v_{m}\right) \\
+\left(a_{3}(y, t) \cdot \nabla v_{m},-\Delta v_{m}\right)+\left(g\left(v_{m}\right),-\Delta v_{m}\right)=\left(f(h(t) y, t),-\Delta v_{m}\right) .
\end{gathered}
$$

Por argumentos análogos as estimativas anteriores, obtemos

$$
\left|\Delta v_{m}\right|^{2} \leq C_{17}
$$

De (2.9), (2.15) e (2.17), temos

$$
\left(v_{m}\right) \text { é limitado em } L^{\infty}\left(\tau, T ; H_{0}^{1}(\mathscr{O}),\right.
$$

$$
\left(v_{m}^{\prime}\right) \text { é limitado em } L^{\infty}\left(\tau, T ; L^{2}(\mathscr{O})\right)
$$

$\left(v_{m}^{\prime}\right)$ é limitado em $L^{\infty}\left(\tau, T ; H_{0}^{1}(\mathscr{O})\right)$

$\left(v_{m}^{\prime \prime}\right)$ é limitado em $L^{\infty}\left(\tau, T ; L^{2}(\mathscr{O})\right)$,

$\left(\Delta v_{m}\right)$ é limitado em $L^{\infty}\left(\tau, T ; L^{2}(\mathscr{O})\right)$. 


\section{Passagem ao limite:}

De (2.18), (2.19), (2.20), (2.21) e (2.22), temos que

$$
\begin{aligned}
& v_{m} \rightarrow v \text { fraco estrela em } L^{\infty}\left(\tau, T ; H_{0}^{1}(\mathscr{O})\right), \\
& v_{m}^{\prime} \rightarrow v^{\prime} \text { fraco estrela em } L^{\infty}\left(\tau, T ; H_{0}^{1}(\mathscr{O})\right), \\
& v_{m}^{\prime \prime} \rightarrow v^{\prime \prime} \text { fraco estrela em } L^{\infty}\left(\tau, T ; L^{2}(\mathscr{O})\right),
\end{aligned}
$$

$$
\Delta v_{m} \rightarrow \Delta v_{m} \text { fraco estrela em } L^{\infty}\left(\tau, T ; L^{2}(\mathscr{O})\right)
$$

Por outro lado, como a imersão $H_{0}^{1}(\mathscr{O}) \hookrightarrow L^{2}(\mathscr{O})$ é compacta, segue do Teorema de AubinLions que o espaço

$$
W=\left\{v \in L^{2}\left(\tau, T ; H_{0}^{1}(\mathscr{O})\right) ; \quad v^{\prime} \in L^{2}\left(\tau, T ; L^{2}(\mathscr{O})\right)\right\}
$$

munido da norma

$$
\|v\|_{W}=\|v\|_{L^{2}\left(\tau, T ; H_{0}^{1}(\mathscr{O})\right)}+\left\|v^{\prime}\right\|_{L^{2}\left(\tau, T ; L^{2}(\mathscr{O})\right)}
$$

tem imersão compacta em $L^{2}\left(\tau, T ; L^{2}(\mathscr{O})\right)$. Como $\left(v_{m}\right)$ é limitada em $W$, temos que existe uma subsequência de $\left(v_{m}\right)$, a qual continuaremos chamando de $v_{m}$, tal que

$$
\left(v_{m}\right) \rightarrow v \text { forte em } L^{2}\left(\tau, T ; L^{2}(\mathscr{O})\right)
$$


Com as convergências (2.23), (2.24), (2.25), (2.26) e (2.27) podemos passar o limite na equação aproximada (2.5). Como $\left(w_{k}\right)_{k \in \mathbb{N}}$ é denso em $H_{0}^{1}(\mathscr{O})$ podemos substituir em (2.5) $w_{k}$ por $w \in H_{0}^{1}(\mathscr{O})$, multiplicando por $\varphi \in \mathscr{D}(\tau, T)$ e integrando de $\tau$ até $T$, obtemos

$$
\begin{aligned}
& \int_{\tau}^{T}\left(v_{m}^{\prime \prime}, w\right) \varphi d t+\int_{\tau}^{T} \eta\left(v_{m}^{\prime}, w\right) \varphi d t+\int_{\tau}^{T} a\left(t, v_{m}, w\right) \varphi d t+\int_{\tau}^{T}\left(a_{2}(y, t) \cdot \nabla v_{m}^{\prime}, w\right) \varphi d t \\
& \quad+\int_{\tau}^{T}\left(a_{3}(y, t) \cdot \nabla v_{m}, w\right) \varphi d t+\int_{\tau}^{T}\left(g\left(v_{m}\right), w\right) \varphi d t=\int_{\tau}^{T}(f(h(t) y, t), w) \varphi d t .
\end{aligned}
$$

Das convergências (2.23), (2.24), (2.25), (2.26), (2.27) e fazendo $m \rightarrow \infty$ temos que

$$
\begin{aligned}
& \int_{\tau}^{T}\left(v^{\prime \prime}, w\right) \varphi d t+\int_{\tau}^{T} \eta\left(v^{\prime}, w\right) \varphi d t+\int_{\tau}^{T} a(t, v, w) \varphi d t+\int_{\tau}^{T}\left(a_{2}(y, t) \cdot \nabla v^{\prime}, w\right) \varphi d t \\
& \quad+\int_{\tau}^{T}\left(a_{3}(y, t) \cdot \nabla v, w\right) \varphi d t+\int_{\tau}^{T}(g(v), w) \varphi d t=\int_{\tau}^{T}(f(h(t) y, t), w) \varphi d t .
\end{aligned}
$$

Considerando em particular $w \in \mathscr{D}(\mathscr{O})$, temos

$$
\int_{\tau}^{T}\left\langle v^{\prime \prime}+\eta v^{\prime}+A(t) v+a_{2}(y, t) \cdot \nabla v^{\prime}+a_{3}(y, t) \cdot \nabla v+g(v)-f(h(t) y, t), w \varphi\right\rangle d t=0 .
$$

Assim

$$
\left\langle v^{\prime \prime}+\eta v^{\prime}+A(t) v+a_{2}(y, t) \cdot \nabla v^{\prime}+a_{3}(y, t) \cdot \nabla v+g(v)-f(h(t) y, t), \phi\right\rangle=0
$$

para todo $\phi \in \mathscr{D}^{\prime}((\tau, T) \times(\mathscr{O}))$.

\section{Segue portanto}

$$
v^{\prime \prime}+\eta v^{\prime}+A(t) v+a_{2}(y, t) \cdot \nabla v^{\prime}+a_{3}(y, t) \cdot \nabla v+g(v)=f(h(t) y, t) \text { em } \mathscr{D}^{\prime}\left(\mathscr{Q}_{\tau, T}\right) .
$$

Desde que $v^{\prime \prime}(t) \in L^{2}(\mathscr{O}), f(h(t) y, t) \in L^{2}(\mathscr{O}), \nabla v^{\prime}(t) \in L^{2}(\mathscr{O}), \nabla v(t) \in L^{2}(\mathscr{O})$, concluímos $\Delta v(t) \in$ $L^{2}(\mathscr{O})$. Assim,

$$
v^{\prime \prime}+\eta v^{\prime}+A(t) v+a_{2}(y, t) \cdot \nabla v^{\prime}+a_{3}(y, t) \cdot \nabla v+g(v)=f(h(t) y, t)
$$

em $L^{2}(\mathscr{O})$, para todo $t \in[\tau, T]$. 
Além disso, usando o resultado de regularidade de problemas elípticos temos $v(t) \in H_{0}^{1}(\mathscr{O}) \cap$ $H^{2}(\mathscr{O})$, para todo $t \in[\tau, T]$. Deste fato e da última igualdade obtemos que $v \in C\left(\tau, T ; H_{0}^{1}(\mathscr{O}) \cap\right.$ $\left.H^{2}(\mathscr{O})\right)$.

\section{Condições iniciais:}

Mostraremos que $v(\tau)=v_{\tau 0}$ e que $v^{\prime}(\tau)=v_{\tau 1}$.

1. $v(\tau)=v_{\tau 0}$.

Faz sentido calcularmos $v_{\tau}$ pois $v \in L^{\infty}\left(\tau, T ; H_{0}^{1}(\mathscr{O}) \cap H^{2}(\mathscr{O})\right)$ e $v^{\prime} \in L^{\infty}\left(\tau, T ; H_{0}^{1}(\mathscr{O})\right)$, logo $v \in C^{0}\left(\tau, T ; H_{0}^{1}\right)$

Dado $\theta \in C^{1}([\tau, T]) \operatorname{com} \theta(\tau)=1$ e $\theta(T)=0$, segue de (2.24) que

$$
\int_{\tau}^{T}\left(v_{m}^{\prime}(t), w\right) \theta(t) d t \rightarrow \int_{\tau}^{T}\left(v^{\prime}(t), w\right) \theta(t) d t \quad \forall w \in H_{0}^{1}(\mathscr{O})
$$

Podemos reescrever (2.30) da seguinte forma

$$
\int_{\tau}^{T} \frac{d}{d t}\left(v_{m}(t), w\right) \theta(t) d t \rightarrow \int_{\tau}^{T} \frac{d}{d t}(v(t), w) \theta(t) d t
$$

Integrando por partes $(2.31)$, resulta

$$
-\int_{\tau}^{T}\left(v_{m}(t), w\right) \theta^{\prime}(t) d t-\left(v_{0 \tau m}, w\right) \rightarrow-\int_{\tau}^{T}(v(t), w) \theta^{\prime}(t) d t-(v(\tau), w)
$$

Por (2.23), temos

$$
\int_{\tau}^{T}\left(v_{m}(t), w\right) \theta^{\prime}(t) d t \rightarrow \int_{\tau}^{T}(v(t), w) \theta^{\prime}(t) d t
$$

Utilizando (2.33) e (2.32), resulta

$$
\left(v_{0 \tau m}, w\right) \rightarrow(v(\tau), w) \text { para todo } w \in H_{0}^{1}(\mathscr{O})
$$


Por outro lado segue de $(2.5)_{3}$

$$
\left(v_{0 \tau m}, w\right) \rightarrow\left(v_{\tau 0}, w\right) \text { para todo } w \in H_{0}^{1}(\mathscr{O})
$$

Portanto, de (2.35) e (2.34), concluímos que

$$
v(\tau)=v_{\tau 0}
$$

2. $v^{\prime}(\tau)=v_{\tau 1}$

Segue analogamente ao item 1.

\subsubsection{Existência e unicidade de solução forte para o sistema (2.2)}

O resultado principal é dado pelo teorema abaixo:

Teorema 2.1.2 Seja $f \in H_{\text {loc }}^{1}\left(\mathbb{R}, L^{2}\left(\mathscr{O}_{t}\right)\right)$, sobre as hipóteses $(H .1)$, (H.2), (H.3). Então, se $u_{\tau 0} \in$ $H_{0}^{1}\left(\mathscr{O}_{\tau}\right) \cap H^{2}\left(\mathscr{O}_{\tau}\right)$ e $u_{\tau 1} \in H_{0}^{1}\left(\mathscr{O}_{\tau}\right)$, existe uma única função $u: \widehat{\mathscr{Q}_{\tau, T}} \longrightarrow \mathbb{R}$ que é solução forte do problema (2.2), isto é,

$$
u \in L^{\infty}\left(\tau, T ; H_{0}^{1}\left(\mathscr{O}_{t}\right) \cap H^{2}\left(\mathscr{O}_{t}\right)\right) ; \quad \frac{\partial u}{\partial t} \in L^{\infty}\left(\tau, T ; H_{0}^{1}\left(\mathscr{O}_{t}\right)\right) e \frac{\partial^{2} u}{\partial t^{2}} \in L^{\infty}\left(\tau, T ; L^{2}\left(\mathscr{O}_{t}\right)\right)
$$

\section{Demonstração:}

\section{Existência:}

Daremos uma idéia da demostração para o teorema 2.1.2. O Teorema 2.1.1 garante que existe função $v$ solução do problema (2.3). como $r$ é um difeomorfismo temos que $u$ é uma solução para (2.2). 
De fato, seja $v$ a solução do sistema (2.3) obtido do Teorema 2.1.1, com condições iniciais

$$
v_{\tau 0}(y)=u_{\tau 0}(h(\tau) y), \quad v_{\tau 1}(y)=u_{\tau 1}(h(\tau) y)
$$

Considere a função $u(x, t)=v(y, t)$ onde $x=h(t) y$.

Vamos mostrar que $u(x, t)$, sobre a hipóteses do teorema (2.1.2), é solução do problema (2.2). Para isto é suficiente mostrar que a solução $v$ do problema (2.3) quando transformado pela aplicação $r^{-1}$ resulta na solução do sistema (2.2) e, portanto, a classe de soluções do Teorema 2.1.1 resultam nas classes de soluções do Teorema 2.1.2.

De fato, temos que

1. $\Delta u(x, t)=\frac{1}{h^{2}(t)} \Delta v$,

2. $\frac{\partial^{2} u}{\partial t^{2}}=\frac{\partial^{2} v}{\partial t^{2}}(y, t)+A(t) v(y, t)+a_{2}(y, t) \cdot \nabla \frac{\partial v}{\partial t}(y, t)+a_{3}(y, t) \cdot \nabla v$.

Pelo sistema (2.3) temos que $u$ satisfaz o sistema (2.2).

A regularidade de $v(y, t)$ dado pelo teorema (2.1.1) implica que $u(x, t)$ é solução do sistema (2.2).

Primeiro de tudo, para demonstrar a unicidade, introduziremos um lema útil para um domínio não cilíndrico, provado em Bardos e Chen [1].

Lema 2.1.3 Suponhamos que a fronteira lateral $\widehat{\Sigma} e ́$ regular e $v=\left(v_{x}, v_{t}\right)$ o seu vetor normal unitário.

- Suponha u regular em $\widehat{\mathscr{Q}}$ e tal que $u=0$ sobre $\widehat{\Sigma}$. Então

$$
\frac{\partial u}{\partial t}=\frac{\partial u}{\partial v} v_{t} \quad e \quad \nabla u=\frac{\partial u}{\partial v} v_{x}
$$

- Seja $F: \mathscr{O}_{t} \times[\tau, T] \longrightarrow \mathbb{R}$ uma função diferenciável. Então,

$$
\frac{d}{d t} \int_{\mathscr{O}_{t}} F(x, t) d x=\int_{\mathscr{O}_{t}} \frac{\partial}{\partial t} F(x, t) d x-\int_{\partial \mathscr{O}_{t}} F(x, t) v_{t} d \sigma
$$




\section{Unicidade:}

Suponhamos que $u_{i}(t)(i=1,2)$ sejam duas soluções do problema (2.2), com condição inicial $\left(u_{\tau 0}, u_{\tau 1}\right) \in H_{0}^{1}\left(\mathscr{O}_{\tau}\right) \cap H^{2}\left(\mathscr{O}_{\tau}\right) \times H_{0}^{1}\left(\mathscr{O}_{\tau}\right)$. Fazendo $w(t)=u_{1}(t)-u_{2}(t)$ a diferença das respectivas soluções, temos que $w$ satisfaz o seguinte problema

$$
\left\{\begin{array}{l}
\frac{\partial^{2} w}{\partial t^{2}}+\eta \frac{\partial w}{\partial t}-\Delta w=g\left(u_{2}\right)-g\left(u_{1}\right) \text { em } \widehat{\mathscr{Q}}_{\tau, T} \\
w=0 \text { sobre } \widehat{\Sigma}_{\tau, T} \\
w(x, \tau)=\frac{\partial w}{\partial t}(x, \tau)=0 \text { para } x \in \mathscr{O}_{\tau}
\end{array}\right.
$$

Multiplicando (2.39) por $w^{\prime}(\theta)$ temos

$$
\frac{1}{2} \frac{\partial}{\partial t}\left(\frac{\partial w}{\partial t}\right)^{2}+\frac{1}{2} \frac{\partial}{\partial t}|\nabla w|^{2}+\left(\frac{\partial w}{\partial t}\right)^{2}=\operatorname{div}\left(\nabla w \frac{\partial w}{\partial t}\right)+\left(g\left(u_{2}\right)-g\left(u_{1}\right)\right) \frac{\partial w}{\partial t}
$$

Integrando sobre $\Omega_{t}$ obtemos, utilizando o teorema da divergência e a identidade (2.38),

$$
\begin{gathered}
\frac{1}{2} \frac{d}{d \theta}\left|w^{\prime}(\theta)\right|_{\theta}^{2}+\frac{1}{2} \frac{d}{d \theta} \|\left. w(\theta)\right|_{\theta} ^{2}+\eta \int_{\mathscr{O}_{\theta}}\left|w^{\prime}(\theta)\right|^{2} d x \\
=\int_{\mathscr{O}_{\theta}}\left(g\left(u_{2}\right)-g\left(u_{1}\right)\right) w^{\prime}(\theta) d x+R,
\end{gathered}
$$

onde

$$
R=\frac{1}{2} \int_{\partial \mathscr{O}_{\theta}}\left(\left|w^{\prime}(\theta)\right|^{2}+|\nabla w(\theta)|^{2}\right) d \sigma+\int_{\mathscr{O}_{\theta}} \operatorname{div}\left(\nabla w w^{\prime}\right) d x .
$$

Agora de (2.37) vemos que

$$
\frac{1}{2} \int_{\partial \mathscr{O}_{\theta}}\left(\left|w^{\prime}(\theta)\right|^{2}+|\nabla w(\theta)|^{2}\right) d \sigma=\frac{1}{2} \int_{\partial \mathscr{O}_{\theta}}\left(\frac{\partial w}{\partial v}\right)^{2}\left(\left|v_{x}\right|^{2}+\left|v_{t}\right|^{2}\right) v_{t} d \sigma
$$

e

$$
\int_{\mathscr{O}_{\theta}} \operatorname{div}\left(\nabla w w^{\prime}\right) d x=-\int_{\partial \mathscr{O}_{\theta}}\left(\frac{\partial w}{\partial v}\right)^{2}\left|v_{x}\right|^{2} v_{t} d \sigma
$$

Assim

$$
R=\frac{1}{2} \int_{\partial \mathscr{O}_{\theta}}\left(\frac{\partial w}{\partial v}\right)^{2}\left(\left|v_{t}\right|^{2}-\left|v_{x}\right|^{2}\right) v_{t} d \sigma \leq 0
$$

uma vez que a hipótese (H.2) implica que $\left|v_{t}\right|<\left|v_{x}\right|$ (time-like). 
Resulta que

$$
\frac{d}{d \theta}\left(\left|w^{\prime}(\theta)\right|_{\theta}^{2}+\left.|| w(\theta)\right|_{\theta} ^{2}\right) \leq 2 \int_{\mathscr{O}_{\theta}}\left|g\left(u_{2}\right)-g\left(u_{1}\right)\right|\left|w^{\prime}(\theta)\right| d x
$$

Das hipóteses (H.1), (H.3) e (2.40), temos

$$
\frac{d}{d \theta}\left(\left|w^{\prime}(\theta)\right|_{\theta}^{2}+\|w(\theta)\|_{\theta}^{2}\right) \leq C_{1}^{\prime}\left(\left|w^{\prime}(\theta)\right|_{\theta}^{2}+\|w(\theta)\|_{\theta}^{2}\right) .
$$

Integrando (2.41) de $\tau$ até $t$, obtemos

$$
\left|w^{\prime}(t)\right|_{t}^{2}+\|w(t)\|_{t}^{2} \leq \int_{\tau}^{t} C_{1}^{\prime}\left(\left|w^{\prime}(\theta)\right|_{\theta}^{2}+\|w(\theta)\|_{\theta}^{2}\right) d \theta
$$

Finalmente, utilizando a desigualdade de Gronwall, segue

$$
\left|w^{\prime}(t)\right|_{t}=\|w(t)\|_{t}=0 \text { para todo } t \in[\tau, T] .
$$

Assim, $w=0$, portanto $u_{1}=u_{2}$.

\subsection{Existência e unicidade de solução fraca para o sistema auxiliar (2.2)}

Nosso objetivo nesta seção é obter solução para o problema (2.2) com dados inicias menos regulares, isto é, para $u_{\tau 0} \in H_{0}^{1}\left(\mathscr{O}_{\tau}\right)$ e $u_{\tau 1} \in L^{2}\left(\mathscr{O}_{\tau}\right)$. A correspondente solução será chamada de solução fraca. Para obtermos esta solução, aproximaremos $u_{\tau 0}$ e $u_{\tau 1}$ por sequências de vetores em $H_{0}^{1}\left(\mathscr{O}_{\tau}\right) \cap H^{2}\left(\mathscr{O}_{\tau}\right)$ e em $H_{0}^{1}\left(\mathscr{O}_{\tau}\right)$, respectivamente, e aplicaremos o resultado do Teorema 2.1.2 (solução forte). 


\subsubsection{Existência de solução fraca para o sistema auxiliar (2.2)}

Antes de apresentarmos qualquer resultado vamos deixar claro o que entendemos por solução fraca do problema (2.2).

Denotemos por

$$
\mathscr{U}_{\tau, T}:=\left\{\varphi \in L^{2}\left(\tau, T ; H_{0}^{1}\left(\mathscr{O}_{t}\right)\right), \varphi^{\prime} \in L^{2}\left(\tau, T ; L^{2}\left(\mathscr{O}_{t}\right)\right), \varphi(\tau)=\varphi(T)=0\right\}
$$

Definição 2.2.1 (Solução fraca do problema (2.2)) Sejam $\left(u_{\tau 0}, u_{\tau 1}\right) \in H_{0}^{1}\left(\mathscr{O}_{\tau}\right) \times L^{2}\left(\mathscr{O}_{\tau}\right), f \in$ $H^{1}\left(\tau, T ; H^{-1}\left(\mathscr{O}_{t}\right)\right) e-\infty<\tau \leq T<\infty$ dados. Dizemos que a função u é uma solução fraca do problema (1.2), no intervalo $[\tau, T]$, se

1. $u \in C\left([\tau, T] ; H_{0}^{1}\left(\mathscr{O}_{t}\right)\right) \cap C^{1}\left([\tau, T] ; L^{2}\left(\mathscr{O}_{t}\right)\right) \operatorname{com}\left(u(\tau), u^{\prime}(\tau)\right)=\left(u_{\tau 0}, u_{\tau 1}\right)$;

2. Existem sequências $\left.\left(u_{0 \tau \tau}, u_{1 \tau m}\right) \in H_{0}^{1}\left(\mathscr{O}_{\tau}\right) \cap H^{2}\left(\mathscr{O}_{\tau}\right)\right) \times H_{0}^{1}\left(\mathscr{O}_{\tau}\right) e f_{m} \in L^{2}\left(\tau, T ; L^{2}\left(\mathscr{O}_{t}\right)\right), m=$ $1,2, \cdots$, tais que

$$
\begin{gathered}
\left(u_{0 \tau m}, u_{1 \tau m}\right) \rightarrow\left(u_{0 \tau}, u_{1 \tau}\right), \text { em } H_{0}^{1}\left(\mathscr{O}_{\tau}\right) \times L^{2}\left(\mathscr{O}_{\tau}\right), \\
f_{m} \rightarrow f \text { em } L^{2}\left(\tau, T ; H^{-1}\left(\mathscr{O}_{t}\right)\right), \\
u_{m} \rightarrow \text { u em } C\left([\tau, T] ; H_{0}^{1}\left(\mathscr{O}_{t}\right)\right) \text { e } u_{m}^{\prime} \rightarrow u^{\prime} \text { em } C\left([\tau, T] ; L^{2}\left(\mathscr{O}_{t}\right)\right)
\end{gathered}
$$

onde $u_{m}$ é a única solução forte de (2.2) correspondente a $\left(u_{0 \tau m}, u_{1 \tau m}, f_{m}\right)$;

3. Para todo $\varphi \in \mathscr{U}_{\tau, T}$, temos

$$
\begin{aligned}
& -\int_{\tau}^{T} \int_{\mathscr{O}_{t}} u^{\prime}(x, t) \varphi^{\prime}(x, t) d x d t+\int_{\tau}^{T} \int_{\mathscr{O}_{t}} \eta u^{\prime}(x, t) \varphi(x, t) d x d t+\int_{\tau}^{T} \int_{\mathscr{O}_{t}} \nabla u \nabla \varphi d x d t \\
& =-\int_{\tau}^{T} \int_{\mathscr{O}_{t}} g(u(x, t)) \varphi(x, t) d x d t+\int_{\tau}^{T} \int_{\mathscr{O}_{t}} f(x, t) \varphi(x, t) d x d t .
\end{aligned}
$$

O seguinte resultado estabelece a existência de solução fraca para o problema (2.2) no sentido da definição anterior. 
Teorema 2.2.2 Seja $f \in H_{\text {loc }}^{1}\left(\mathbb{R}, L^{2}\left(\mathscr{O}_{t}\right)\right)$, sobre as hipóteses (H.1), (H.2), (H.3). Então, se $u_{0 \tau} \in$ $H_{0}^{1}\left(\mathscr{O}_{\tau}\right), u_{1 \tau} \in L^{2}\left(\mathscr{O}_{\tau}\right)$ e qualquer $-\infty<\tau \leq T<\infty$, existe uma única função $u: \widehat{\mathscr{Q}_{\tau, T}} \longrightarrow \mathbb{R}$, que é solução fraca do problema (2.2).

Demonstração: (Existência) Como $D(-\Delta)$ é denso em $H_{0}^{1}\left(\mathscr{O}_{\tau}\right)$, cf em Lions [16], segue que existe uma sequência $\left(u_{0 \tau m}\right) \subset D(-\Delta) \subset H_{0}^{1}\left(\mathscr{O}_{\tau}\right) \cap H^{2}\left(\mathscr{O}_{\tau}\right)$ tal que

$$
u_{0 \tau m} \rightarrow u_{0 \tau} \text { em } H_{0}^{1}\left(\mathscr{O}_{\tau}\right)
$$

Por outro lado, uma vez que $H_{0}^{1}\left(\mathscr{O}_{\tau}\right)$ é denso em $L^{2}\left(\mathscr{O}_{\tau}\right)$, dado $u_{1 \tau} \in L^{2}\left(\mathscr{O}_{\tau}\right)$, existe uma sequência $\left(u_{1 \tau m}\right) \subset H_{0}^{1}\left(\mathscr{O}_{\tau}\right)$ tal que

$$
u_{1 \tau m} \rightarrow u_{\tau 1} \text { em } L^{2}\left(\mathscr{O}_{\tau}\right)
$$

Por fim, sabemos que $H^{1}\left(\widehat{\mathscr{Q}}_{\tau, T}\right)$ é denso em $L^{2}\left(\widehat{\mathscr{Q}}_{\tau, T}\right)$. Logo, dado $f \in L^{2}\left(\widehat{\mathscr{Q}}_{\tau, T}\right)$ existe uma sequência $\left(f_{m}\right) \subset H^{1}\left(\widehat{\mathscr{Q}}_{\tau, T}\right)$ tal que

$$
f_{m} \rightarrow f \text { em } L^{2}\left(\widehat{\mathscr{Q}}_{\tau, T}\right)
$$

Para cada termo $\left(u_{0 \tau \tau}, u_{1 \tau m}, f_{m}\right) \in\left[H_{0}^{1}\left(\mathscr{O}_{\tau}\right) \cap H^{2}\left(\mathscr{O}_{\tau}\right)\right] \times H_{0}^{1}\left(\mathscr{O}_{\tau}\right) \times H^{1}\left(\tau, T ; L^{2}\left(\mathscr{O}_{t}\right)\right)$, existe uma única função $u_{m}: \widehat{\mathscr{Q}}_{\tau, T} \longrightarrow \mathbb{R}$ satisfazendo as condições do Teorema 2.1.2, isto é,

$$
\left\{\begin{array}{l}
u_{m}^{\prime \prime}+\eta u_{m}^{\prime}-\Delta u_{m}+g\left(u_{m}\right)=f_{m}(t) \text { em } \widehat{\mathscr{Q}}_{\tau, T} \\
u_{m}=0 \text { sobre } \widehat{\Sigma}_{\tau, T} \\
u_{m}(\tau)=u_{0 \tau m}, \quad u_{m}^{\prime}(\tau)=u_{1 \tau m}, \text { em } \mathscr{O}_{\tau}
\end{array}\right.
$$

tal que $u_{m} \in C\left([\tau, T] ; H_{0}^{1}\left(\mathscr{O}_{t}\right) \cap H^{2}\left(\mathscr{O}_{t}\right)\right), u_{m}^{\prime} \in C\left([\tau, T] ; H_{0}^{1}\left(\mathscr{O}_{t}\right)\right)$ e $u_{m}^{\prime \prime} \in C\left([\tau, T] ; L^{2}\left(\mathscr{O}_{t}\right)\right)$. 
Dados $m, n \in \mathbb{N}$, com $m>n$, denotemos $z_{m n}=u_{m}-u_{n}$. Então de (2.47), temos que

$$
\left\{\begin{array}{l}
z_{m n}^{\prime \prime}+\eta z_{m n}^{\prime}-\Delta z_{m n}+g\left(u_{m}\right)-g\left(u_{n}\right)=f_{m}(t)-f_{n}(t) \text { em } \widehat{\mathscr{Q}}_{\tau, T}, \\
z_{m n}(\tau)=u_{0 \tau m}-u_{0 \tau n}, \quad z_{m n}^{\prime}(\tau)=u_{1 \tau m}-u_{1 \tau n}, \text { em } \mathscr{O}_{\tau}
\end{array}\right.
$$

onde $z_{m n} \in C\left([\tau, T] ; H_{0}^{1}\left(\mathscr{O}_{t}\right) \cap H^{2}\left(\mathscr{O}_{t}\right)\right), z_{m n}^{\prime} \in C\left([\tau, T] ; H_{0}^{1}\left(\mathscr{O}_{t}\right)\right)$ e $z_{m n}^{\prime \prime} \in C\left([\tau, T] ; L^{2}\left(\mathscr{O}_{t}\right)\right)$.

Multiplicando $(2.48)_{1}$ por $z_{m n}^{\prime}$ e integrando sobre $\mathscr{O}_{t}$, obtemos

$$
\frac{1}{2} \frac{d}{d t}\left[\left|z_{m n}^{\prime}(t)\right|_{t}^{2}+|| z_{m n}(t)||_{t}^{2}\right]+\eta\left|z_{m n}^{\prime}\right|^{2} \leq\left(g\left(u_{n}\right)-g\left(u_{m}\right), z_{m}^{\prime}\right)_{t}+\left(f_{m}-f_{n}, z_{m n}^{\prime}\right)_{t}
$$

Utilizando (H.1), (H.3), a desigualdade de Hölder generalizada e as imersões de Sobolev, temos

$$
\left(g\left(u_{n}\right)-g\left(u_{m}\right), z_{m}^{\prime}\right)_{t} \leq C_{2}^{\prime}\left(\left|z_{m n}^{\prime}\right|_{t}^{2}+\|\left. z_{m n}\right|_{t} ^{2}\right)
$$

Assim, substituindo em (2.49) obtemos

$$
\frac{d}{d t}\left[\left|z_{m n}^{\prime}(t)\right|_{t}^{2}+\left\|z_{m n}(t)\right\|_{t}^{2}\right] \leq\left\|f_{m}(t)-f_{n}(t)\right\|_{L^{2}\left(\mathscr{O}_{t}\right)}^{2}+C_{3}^{\prime}\left(\left|z_{m n}^{\prime}(t)\right|_{t}^{2}+\left\|z_{m n}(t)\right\|_{t}^{2}\right)
$$

Integrando (2.50) de $\tau$ a $t$, temos

$$
\begin{aligned}
\left|z_{m n}^{\prime}(t)\right|_{t}^{2}+\left\|z_{m n}(t)\right\|_{t}^{2} \leq & \left|u_{1 \tau m}-u_{1 \tau n}\right|_{\tau}^{2}+\left\|u_{0 \tau m}-u_{0 \tau n}\right\|_{\tau}^{2}+\int_{\tau}^{t}\left\|f_{m}(s)-f_{n}(s)\right\|_{L^{2}\left(\mathscr{O}_{s}\right)}^{2} d s \\
& +C_{3}^{\prime} \int_{\tau}^{t}\left(\left|z_{m n}^{\prime}(s)\right|_{s}^{2}+\left\|z_{m n}(s)\right\|_{s}^{2}\right) d s
\end{aligned}
$$

para todo $t \in[\tau, T]$.

Pelo Lema de Gronwall, resulta que

$\left|z_{m n}^{\prime}(t)\right|_{t}^{2}+\|\left. z_{m n}(t)\right|_{t} ^{2} \leq\left(\left|u_{1 \tau m}-u_{1 \tau n}\right|_{\tau}^{2}+\left\|u_{0 \tau m}-u_{0 \tau n}\right\|_{\tau}^{2}+\int_{\tau}^{t}\left\|f_{m}(s)-f_{n}(s)\right\|_{L^{2}\left(\mathscr{O}_{s}\right)}^{2} d s\right) e^{C_{3}^{\prime}(T-\tau)}$

Tomando o máximo, temos

$$
\max _{t \in[\tau, T]}\left|u_{m}^{\prime}(t)-u_{n}^{\prime}(t)\right|_{t}^{2} \leq C_{4}^{\prime}\left(\left|u_{1 \tau m}-u_{1 \tau n}\right|_{\tau}^{2}+\left\|u_{0 \tau m}-\left.u_{0 \tau n}\right|_{\tau} ^{2}+\int_{\tau}^{t}\right\| f_{m}(s)-f_{n}(s) \|_{L^{2}\left(\mathscr{O}_{s}\right)}^{2} d s\right)
$$


onde $C_{4}^{\prime}=e^{C_{3}^{\prime}(T-\tau)}$.

Fazendo $m, n \rightarrow \infty$, obtemos

$$
\left\|u_{m}^{\prime}(t)-u_{n}^{\prime}(t)\right\|_{C\left([\tau, T] ; L^{2}\left(\mathscr{O}_{t}\right)\right)} \rightarrow 0
$$

Logo, $\left(u^{\prime}\right)_{m \in \mathbb{N}}$ é de Cauchy em $C\left([\tau, T] ; L^{2}\left(\mathscr{O}_{t}\right)\right)$.

Da mesma forma, mostramos também que $(u)_{m \in \mathbb{N}}$ é de Cauchy em $C\left([\tau, T] ; H_{0}^{1}\left(\mathscr{O}_{t}\right)\right)$. Sendo os espaços envolvidos de Banach, resulta que

$$
\begin{gathered}
u_{m} \rightarrow u \text { em } C\left([\tau, T] ; H_{0}^{1}\left(\mathscr{O}_{t}\right)\right) \hookrightarrow L^{2}\left(\tau, T ; H_{0}^{1}\left(\mathscr{O}_{t}\right)\right) \hookrightarrow \mathscr{D}^{\prime}\left(\tau, T ; H_{0}^{1}\left(\mathscr{O}_{t}\right)\right) \\
u_{m}^{\prime} \rightarrow u^{\prime} \text { em } C\left([\tau, T] ; L^{2}\left(\mathscr{O}_{t}\right)\right) \hookrightarrow L^{2}\left(\tau, T ; L^{2}\left(\mathscr{O}_{t}\right)\right) \hookrightarrow \mathscr{D}^{\prime}\left(\tau, T ; L^{2}\left(\mathscr{O}_{t}\right)\right)
\end{gathered}
$$

Compondo $(2.47)_{1}$ por $w \in H_{0}^{1}\left(\mathscr{O}_{t}\right)$, integrando em $\mathscr{O}_{t}$ e em seguida multiplicando por $\theta \in$ $\mathscr{D}(\tau, T)$, tal que $\theta(\tau)=\theta(T)=0$, teremos

$$
\begin{aligned}
& \int_{\tau}^{T} \int_{\mathscr{O}_{t}} u_{m}^{\prime \prime}(x, t) w(x) \theta(t) d x d t+\int_{\tau}^{T} \int_{\mathscr{O}_{t}} \eta u_{m}^{\prime}(x, t) w(x) \theta(t) d x d t-\int_{\tau}^{T} \int_{\mathscr{O}_{t}} \Delta u_{m} w(x) \theta(t) d x d t \\
& =-\int_{\tau}^{T} \int_{\mathscr{O}_{t}} g\left(u_{m}(x, t)\right) w(x) \theta(t) d x d t+\int_{\tau}^{T} \int_{\mathscr{O}_{t}} f(x, t) w(x) \theta(t) d x d t
\end{aligned}
$$

$\mathrm{Na}$ igualdade acima, integrando por partes com respeito à variável $t$ e pelo Teorema de Green, vem que

$$
\begin{aligned}
& -\int_{\tau}^{T} \int_{\mathscr{O}_{t}} u_{m}^{\prime}(x, t) w(x) \theta^{\prime}(t) d x d t+\int_{\tau}^{T} \int_{\mathscr{O}_{t}} \eta u_{m}^{\prime}(x, t) w(x) \theta(t) d x d t+\int_{\tau}^{T} \int_{\mathscr{O}_{t}} \nabla u_{m} \nabla w \theta(t) d x d t \\
& =-\int_{\tau}^{T} \int_{\mathscr{O}_{t}} g\left(u_{m}(x, t)\right) w(x) \theta(t) d x d t+\int_{\tau}^{T} \int_{\mathscr{O}_{t}} f(x, t) w(x) \theta(t) d x d t .
\end{aligned}
$$

Como visto anteriormente, $(u)_{m \in \mathbb{N}}$ é de Cauchy em $C\left([\tau, T] ; H_{0}^{1}\left(\mathscr{O}_{t}\right)\right)$ e $\left(u_{m}^{\prime}\right)$ é de Cauchy em $C\left([\tau, T] ; L^{2}\left(\mathscr{O}_{t}\right)\right)$; o que nos diz que

$$
\left(u_{m}\right) \text { é limitado em } L^{2}\left(\tau, T ; H_{0}^{1}\left(\mathscr{O}_{t}\right)\right) \text {, }
$$




$$
\left(u_{m}^{\prime}\right) \text { é limitado em } L^{2}\left(\tau, T ; L^{2}\left(\mathscr{O}_{t}\right)\right) \text {. }
$$

Logo,

$$
\begin{aligned}
& u_{m} \rightarrow u \text { fraco em } L^{2}\left(\tau, T ; H_{0}^{1}\left(\mathscr{O}_{t}\right)\right), \\
& u_{m}^{\prime} \rightarrow u^{\prime} \text { fraco em } L^{2}\left(\tau, T ; L^{2}\left(\mathscr{O}_{t}\right)\right) .
\end{aligned}
$$

Assim,

$$
\begin{aligned}
\int_{\tau}^{T}\left\langle u_{m}^{\prime}(x, t), w(x)\right\rangle_{t} \theta^{\prime}(t) d t & \rightarrow \int_{\tau}^{T}\left\langle u^{\prime}(x, t), w(x)\right\rangle_{t} \theta^{\prime}(t) d t, \\
\int_{\tau}^{T}\left\langle\eta u_{m}^{\prime}(x, t), w(x)\right\rangle_{t} \theta(t) d t & \rightarrow \int_{\tau}^{T}\left\langle\eta u^{\prime}(x, t), w(x)\right\rangle_{t} \theta(t) d t, \\
\int_{\tau}^{T}\left\langle\nabla u_{m}, \nabla w\right\rangle_{t} \theta(t) d t & \rightarrow \int_{\tau}^{T}\left\langle\frac{\partial u}{\partial x}, \frac{\partial w}{\partial x}\right\rangle_{t} \theta(t) d t .
\end{aligned}
$$

Por outro lado, da convergência (2.51), obtemos

$$
u_{m} \rightarrow u \text { forte em } L^{2}\left(\mathscr{O}_{t} \times(\tau, T)\right)
$$

e portanto, $u_{m} \longrightarrow u$ quase sempre. Do fato que $g$ é contínua, temos

$$
g\left(u_{m}\right) \rightarrow g(u) \text { quase sempre em } \mathscr{O}_{t} \times(\tau, T) .
$$

Mas, por meio da condição de crescimento imposta na hipótese (H.3), obtemos que $\left\|g\left(u_{m}\right)\right\|_{L^{2}\left(\mathscr{O}_{t} \times(\tau, T)\right)}$ é limitada, e pelo Lema de Lions, temos que

$$
g\left(u_{m}\right) \rightarrow g(u) \text { fraco em } L^{2}\left(\mathscr{O}_{t} \times(\tau, T)\right) .
$$


Assim, de (2.57), temos

$$
\int_{\tau}^{T}\left\langle g\left(u_{m}(x, t)\right), w(x)\right\rangle_{t} \theta(t) d t \rightarrow \int_{\tau}^{T}\langle g(u(x, t)), w(x)\rangle_{t} \theta(t) d t
$$

De (2.54), (2.55), (2.56) e (2.58) na passagem ao limite em (2.53), temos

$$
\begin{aligned}
& -\int_{\tau}^{T} \int_{\mathscr{O}_{t}} u^{\prime}(x, t) w(x) \theta^{\prime}(t) d x d t+\int_{\tau}^{T} \int_{\mathscr{O}_{t}} \eta u^{\prime}(x, t) w(x) \theta(t) d x d t+\int_{\tau}^{T} \int_{\mathscr{O}_{t}} \nabla u \nabla w \theta(t) d x d t \\
& =-\int_{\tau}^{T} \int_{\mathscr{O}_{t}} g(u(x, t)) w(x) \theta(t) d x d t+\int_{\tau}^{T} \int_{\mathscr{O}_{t}} f(x, t) w(x) \theta(t) d x d t .
\end{aligned}
$$

Observemos que $\mathscr{U}_{\tau, T}$ munido da norma

$$
\|\varphi\|_{\mathscr{U}_{\tau, T}}=\|\varphi\|_{L^{2}\left(\tau, T ; H_{0}^{1}\left(\mathscr{O}_{t}\right)\right)}+\left\|\varphi^{\prime}\right\|_{L^{2}\left(\tau, T ; L^{2}\left(\mathscr{O}_{t}\right)\right)}
$$

é um espaço de Banach. Além disso,

$$
\left\{(w \cdot \theta) ; w \in H_{0}^{1}\left(\mathscr{O}_{t}\right) \text { e } \theta \in \mathscr{D}(\tau, T)\right\} \subset \mathscr{U}_{\tau, T} \text { é denso em } \mathscr{U}_{\tau, T} .
$$

Então de (2.59), concluímos

$$
\begin{aligned}
& -\int_{\tau}^{T} \int_{\mathscr{O}_{t}} u^{\prime}(x, t) \varphi^{\prime}(x, t) d x d t+\int_{\tau}^{T} \int_{\mathscr{O}_{t}} \eta u^{\prime}(x, t) \varphi(x, t) d x d t+\int_{\tau}^{T} \int_{\mathscr{O}_{t}} \nabla u \nabla \varphi d x d t \\
& =-\int_{\tau}^{T} \int_{\mathscr{O}_{t}} g(u(x, t)) \varphi(x, t) d x d t+\int_{\tau}^{T} \int_{\mathscr{O}_{t}} f(x, t) \varphi(x, t) d x d t
\end{aligned}
$$

para todo $\varphi \in \mathscr{U}_{\tau, T}$.

A unicidade de solução fraca requer um procedimento de regularização e pode ser obtida pelo método padrão de Visik-Ladyzhenkaya, cf. Lions e Magenes [15], Capítulo 3, Seção 8.2.2.

Portanto, a prova do Teorema 2.2.2 fica completa. 


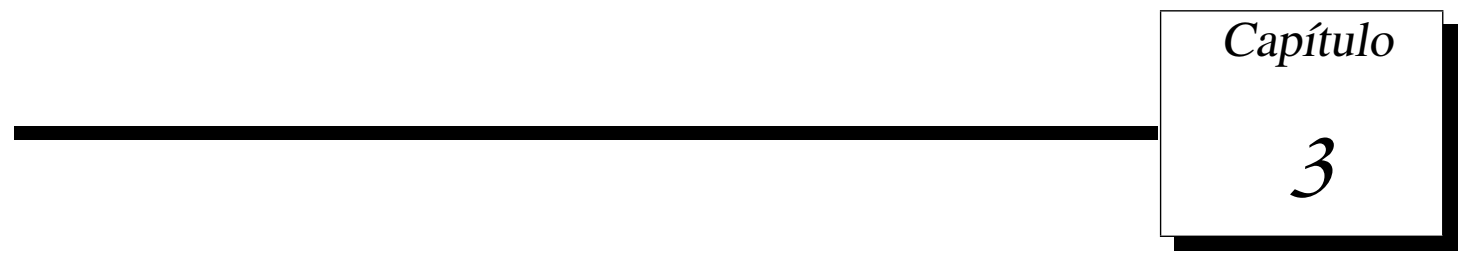

\section{Processo de evolução associado ao problema}

\section{de onda semilinear}

Uma das principais dificuldades no tratamento de problemas envolvendo domínios de fronteira móvel, é que as soluções geralmente são definidas em espaços diferentes a cada tempo $t$. Neste capítulo daremos um tratamento suficientemente adequado para o nosso problema.

\subsection{Definição do processo correspondente}

Para facilitar a leitura reescrevemos o nosso problema de ondas não linear.

$$
\left\{\begin{array}{l}
\frac{\partial^{2} u}{\partial t^{2}}+\eta \frac{\partial u}{\partial t}-\Delta u+g(u)=f(x, t) \text { em } \widehat{\mathscr{Q}}_{\tau}, \\
u=0 \text { sobre } \widehat{\Sigma}_{\tau}, \\
u(x, \tau)=u_{\tau 0}(x), \quad \frac{\partial u}{\partial t}(x, \tau)=u_{\tau 1}(x), x \in \mathscr{O}_{\tau}
\end{array}\right.
$$

onde $\eta>0$,

$$
\widehat{\mathscr{D} \tau}=\bigcup_{t \in(\tau,+\infty)} \mathscr{O}_{t} \times\{t\} \quad \text { e } \quad \widehat{\Sigma}_{\tau}=\bigcup_{t \in(\tau,+\infty)} \partial \mathscr{O}_{t} \times\{t\}
$$

Definição 3.1.1 (Solução fraca) Uma função $u: \bigcup_{t \in[\tau,+\infty)} \mathscr{O}_{t} \times\{t\} \longrightarrow \mathbb{R}$ é chamada solução fraca de (3.1), se para qualquer $T>\tau$, a restrição de u sobre $\bigcup_{t \in[\tau, T)} \mathscr{O}_{t} \times\{t\}$ é solução fraca de (2.2). 
O seguinte teorema garante a existência e unicidade de soluções fracas para o problema (3.1).

Teorema 3.1.2 Com as hipóteses do teorema (2.2.2), para qualquer $u_{0 \tau} \in H_{0}^{1}\left(\mathscr{O}_{\tau}\right), u_{1 \tau} \in L^{2}\left(\mathscr{O}_{\tau}\right)$ e $f \in H_{l o c}^{1}\left(\mathbb{R}, L^{2}\left(\mathscr{O}_{t}\right)\right)$, o problema (3.1) tem uma única solução fraca.

Demonstração: A demonstração segue imediatamente do Teorema 2.2.2.

Definição 3.1.3 (Processo de evolução) Um processo de evolução sobre a família de espaços métricos $\left\{\left(X_{t}, d_{t}\right) ; t \in \mathbb{R}\right\}$ é uma família $\{U(t, \tau) ;-\infty<\tau \leq t<+\infty\}$ de aplicações contínuas $U(t, \tau): X_{\tau} \longrightarrow X_{t}$ com as seguintes propriedades:

1. $U(\tau, \tau) x=x$ para todo $x \in X_{\tau}$,

2. $U(t, \tau)=U(t, s) U(s, \tau)$ para todo $\tau \leq s \leq t$.

No que segue, consideramos o espaço de Banach

$$
X_{t}:=H_{0}^{1}\left(\mathscr{O}_{t}\right) \times L^{2}\left(\mathscr{O}_{t}\right)
$$

munido da norma

$$
\|(u, v)\|_{X_{t}}^{2}=\|u\|_{t}^{2}+|v|_{t}^{2}
$$

Vamos assumir que a função $f \in H_{l o c}^{1}\left(\mathbb{R} ; L^{2}\left(\mathscr{O}_{t}\right)\right)$. Definimos para todo $t \geq \tau$ as aplicações

$$
U(t, \tau): X_{\tau} \longrightarrow X_{t} \text {, para todo }-\infty<\tau \leq t<\infty
$$

tal que

$$
U(t, \tau)\left(u_{0 \tau}, u_{1 \tau}\right):=\left(u\left(t, \tau ;\left(u_{0 \tau}, u_{1 \tau}\right)\right), u^{\prime}\left(t, \tau ;\left(u_{0 \tau}, u_{1 \tau}\right)\right)\right)=\left(u(t), u^{\prime}(t)\right)
$$

onde $u\left(., \tau ;\left(u_{0 \tau}, u_{1 \tau}\right)\right)$ é a única solução fraca de (3.1) obtida pelo Teorema 3.1.2.

Pela existência e unicidade de soluções fracas do problema (3.1), a família de operadores $\{U(t, \tau):-\infty<\tau \leq t<\infty\}$ satisfazem as seguintes propriedades: 


$$
\begin{gathered}
U(\tau, \tau)=I d \text { (identidade em } X_{\tau} \text { ) para todo } \tau \in \mathbb{R}, \\
U(t, s) U(s, \tau)=U(t, \tau) \text { para todo }-\infty<\tau \leq t<\infty .
\end{gathered}
$$

Tendo em conta a Definição 3.1.3, para garantir que as soluções fracas geram um processo de evolução resta mostrar que as aplicações $U(t, \tau): X_{\tau} \longrightarrow X_{t}$ sejam contínuas para cada $\tau \leq t$ fixos.

Lema 3.1.4 Seja $\left(u_{0 \tau}^{i}, u_{1 \tau}^{i}\right) \in X_{\tau} e\left(u^{i}(s), u_{t}^{i}(s)\right)=U(s, \tau)\left(u_{0 \tau}^{i}, u_{1 \tau}^{i}\right),(i=1,2)$.

Então existe $C>0$, tal que

$$
\| u^{1}(t)-\left.u^{2}(t)\right|_{t} ^{2}+\left|u_{t}^{1}(t)-u_{t}^{2}(t)\right|_{t}^{2} \leq e^{C(t-s)}\left(|| u^{1}(s)-u^{2}(s)||_{s}^{2}+\left|u_{t}^{1}(s)-u_{t}^{2}(s)\right|_{s}^{2}\right),
$$

para todo $\tau \leqslant s \leqslant t$.

Demonstração: Fixemos $t>\tau$. Por definição, existem sequências $\left\{\left(u_{0 \tau m}^{i}, u_{1 \tau m}^{i}, f_{m}^{i}\right)\right\}(i=1,2)$ satisfazendo

$$
\begin{gathered}
u_{0 \tau m}^{i} \in H_{0}^{1}\left(\mathscr{O}_{\tau}\right) \cap H^{2}\left(\mathscr{O}_{\tau}\right), \\
u_{1 \tau m}^{i} \in H_{0}^{1}\left(\mathscr{O}_{\tau}\right) \mathrm{e} \\
f_{m}^{i} \in L^{2}\left(\tau, t ; H^{-1}\left(\mathscr{O}_{s}\right)\right)
\end{gathered}
$$

tal que

$$
\begin{gathered}
u_{0 \tau m}^{i} \rightarrow u_{0 \tau}^{i} \text { em } H_{0}^{1}\left(\mathscr{O}_{\tau}\right), \\
u_{1 \tau m}^{i} \rightarrow u_{1 \tau}^{i} \text { em } L^{2}\left(\mathscr{O}_{\tau}\right), \\
f_{m}^{i} \longrightarrow f \text { em } L^{2}\left(\tau, t ; H^{-1}\left(\mathscr{O}_{s}\right)\right) .
\end{gathered}
$$

Temos também

$$
u_{m}^{i} \rightarrow u^{i} \text { em } C^{1}\left([\tau, t] ; L^{2}\left(\mathscr{O}_{s}\right)\right) \cap C\left([\tau, t] ; H_{0}^{1}\left(\mathscr{O}_{s}\right)\right)
$$


onde $u_{m}^{i}$ é a única solução forte correspondente a $\left(u_{0 \tau m}^{i}, u_{1 \tau m}^{i}, f_{m}^{i}\right)$.

Fazendo $w_{m}(\theta)=u_{m}^{1}(\theta)-u_{m}^{2}(\theta)$ a diferença das respectivas soluções, obtemos

$$
w_{m}^{\prime \prime}(\boldsymbol{\theta})+\eta w_{m}^{\prime}(\boldsymbol{\theta})-\Delta w(\boldsymbol{\theta})=g\left(u_{m}^{2}(\boldsymbol{\theta})\right)-g\left(u_{m}^{1}(\boldsymbol{\theta})\right)+f_{m}^{1}(\boldsymbol{\theta})-f_{m}^{2}(\boldsymbol{\theta}) .
$$

Multiplicando (3.3) por $w^{\prime}(\theta)$, temos

$$
\begin{aligned}
\int_{\mathscr{O}_{\theta}} w_{m}^{\prime \prime}(\theta) w_{m}^{\prime}(\theta) d x+\eta \int_{\mathscr{O}_{\theta}} w^{\prime}(\theta)^{2} d x+\int_{\mathscr{O}_{\theta}} \nabla w_{m}(\theta) \nabla w_{m}^{\prime}(\theta) d x= & \int_{\mathscr{O}_{\theta}}\left(g\left(u_{m}^{2}\right)-g\left(u_{m}^{1}\right)\right) w_{m}^{\prime}(\theta) d x \\
& +\int_{\mathscr{O}_{\theta}}\left(f_{m}^{1}(\theta)-f_{m}^{2}(\theta)\right) w^{\prime}(\theta) d x
\end{aligned}
$$

Assim, obtemos

$$
\begin{aligned}
\frac{1}{2} \frac{d}{d \theta}\left(\left\|w_{m}(\theta)\right\|_{\theta}^{2}+\left|w_{m}^{\prime}(\theta)\right|_{\theta}^{2}\right)-\frac{h^{\prime}}{2 h} \int_{\partial \mathscr{O}_{\theta}}\left(\left|w^{\prime}(\boldsymbol{\theta})\right|^{2}+\|w(\theta)\|^{2}\right)(x \cdot \bar{v}) d\left(\partial \mathscr{O}_{\theta}\right) \leq & C\left(\left\|w_{m}(\boldsymbol{\theta})\right\|_{\theta}^{2}+\left|w_{m}^{\prime}\right|_{\theta}^{2}\right) \\
& +\left\|f_{m}^{1}(\boldsymbol{\theta})-f_{m}^{2}(\boldsymbol{\theta})\right\|_{L^{2}\left(\mathscr{O}_{\theta}\right)}^{2}
\end{aligned}
$$

Portanto

$$
\frac{d}{d \theta}\left(\left\|w_{m}(\theta)\right\|_{\theta}^{2}+\left|w_{m}^{\prime}(\boldsymbol{\theta})\right|_{\theta}^{2}\right) \leq C\left(\left\|w_{m}(\boldsymbol{\theta})\right\|_{\theta}^{2}+\left|w_{m}^{\prime}\right|_{\theta}^{2}\right)+\left\|f_{m}^{1}(\boldsymbol{\theta})-f_{m}^{2}(\boldsymbol{\theta})\right\|_{L^{2}\left(\mathscr{O}_{\theta}\right)}^{2}
$$

Integrando de $s$ até $t$, temos

$$
\begin{aligned}
\left\|w_{m}(t)\right\|_{t}^{2}+\left|w_{m}^{\prime}(t)\right|_{t}^{2} & \leq\left\|w_{m}(s)\right\|_{s}^{2}+\left|w_{m}(s)^{\prime}\right|_{s}^{2}+\int_{s}^{t}\left\|f_{m}^{1}(\theta)-f_{m}^{2}(\theta)\right\|_{L^{2}\left(\mathscr{O}_{\theta}\right)}^{2} d \theta \\
& +C \int_{s}^{t}\left(\left\|w_{m}(\theta)\right\|_{\theta}^{2}+\left|w_{m}^{\prime}(\theta)\right|_{\theta}^{2}\right) d \theta
\end{aligned}
$$

Por Gronwall, segue

$$
\|\left. w_{m}(t)\right|_{t} ^{2}+\left|w_{m}^{\prime}(t)\right|_{t}^{2} \leq\left(\| w_{m}(s)||_{s}^{2}+\left|w_{m}^{\prime}(s)\right|_{s}^{2}+\int_{s}^{t}\left|f_{m}^{1}(\theta)-f_{m}^{2}(\theta)\right|_{L^{2}\left(\mathscr{O}_{\theta}\right)}^{2} d \theta\right) e^{C(t-s)}
$$

Fazendo $m \rightarrow \infty$, resulta

$$
\|w(t)\|_{t}^{2}+\left|w^{\prime}(t)\right|_{t}^{2} \leq\left(\|w(s)\|_{s}^{2}+\left|w^{\prime}(s)\right|_{s}^{2}\right) e^{C(t-s)}
$$


Portanto a prova fica completa.

Teorema 3.1.5 Fixados $t \geq \tau$, à aplicação $U(t, \tau): X_{\tau} \longrightarrow X_{t}$ definida por (3.2) é contínua.

Demonstração: Segue imediatamente do lema anterior.

Portanto a família de aplicações contínuas $\{U(t, \tau): t \geq \tau\}$ define um processo de evolução associado ao problema de onda semilinear (3.1). 


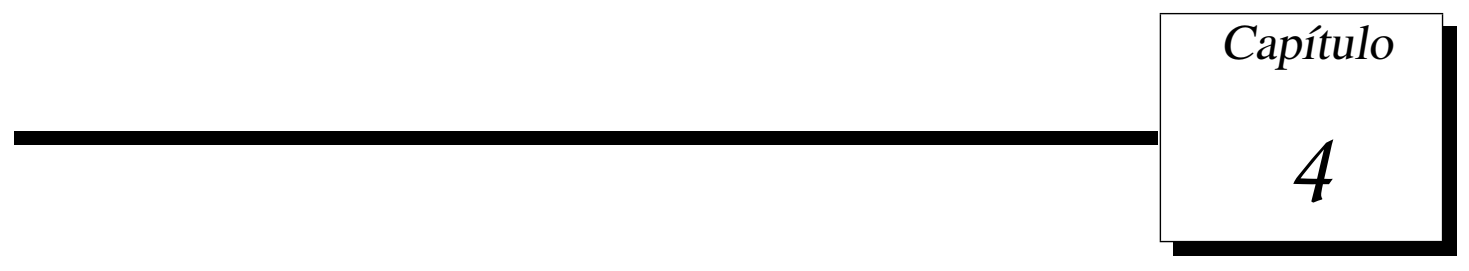

\section{Atrator pullback para o problema de onda} semilinear

Nesta capítulo provamos a nossa principal contribuição, Teorema 4.2.1, sobre a existência de um atrator dependente do tempo correspondente ao problema (3.1). Recordaremos alguns resultados básicos de teoria abstrata de sistemas dinâmicos não autônomos, que são necessárias para o desenvolvimento do estudo.

\subsection{Atratores pullback}

Os resultados nesta seção são variantes dos resultados apresentados em [5] e [13].

A partir de agora consideremos o conjunto $\mathscr{M}=\left\{M ; M: \mathbb{R} \longrightarrow M(t) \in 2^{X_{t}}\right\}$ e escolhemos uma base de atração arbitrária, $\mathscr{D} \subset \mathscr{M}$, como sendo a classe de conjuntos não vazios da forma

$$
\widehat{D}=\left\{D(t) ; D(t) \subset X_{t}, D(t) \neq \emptyset, t \in \mathbb{R}\right\}
$$

Para cada $t \in \mathbb{R}$, denote como $\operatorname{dist}_{t}\left(D_{1}, D_{2}\right)$ a semi-distância de Hausdorff entre subconjuntos não vazios de $X_{t}$, que é definida como

$$
\operatorname{dist}_{t}\left(D_{1}, D_{2}\right)=\sup _{x \in D_{1}} \inf _{y \in D_{2}} d_{t}(x, y) \text { para } D_{1}, D_{2} \subset X_{t}
$$


Definição 4.1.1 (Atrator no sentido $\mathscr{D}$ - pullback) Uma família

$$
\widehat{\mathscr{A}}=\left\{\mathscr{A}(t): \mathscr{A}(t) \subset X_{t}, \mathscr{A}(t) \neq \emptyset, t \in \mathbb{R}\right\}
$$

é chamado atrator no sentido $\mathscr{D}$-pullback associado ao processo $\{U(t, \tau): t \geq \tau\}$ se:

1. $\mathscr{A}(t)$ é um subconjunto compacto de $X_{t}$ para todo $t \in \mathbb{R}$,

2. $\widehat{\mathscr{A}}$ atrai subconjuntos de $\mathscr{D}$ no sentido pullback, ou seja,

$$
\lim _{\tau \rightarrow-\infty} \operatorname{dist}_{t}(U(t, \tau) D(\tau), \mathscr{A}(t))=0, \text { para todo } \widehat{D} \in \mathscr{D} \text { e para todo } t \in \mathbb{R}
$$

3. $\widehat{A}$ é invariante, ou seja

$$
U(t, \tau) \mathscr{A}(\tau)=\mathscr{A}(t) \text { para todo }-\infty<\tau \leq t<+\infty
$$

Os dois conceitos a seguir são essenciais para garantir a existência de atratores no sentido $\mathscr{D}$ pullback.

Definição 4.1.2 (Conjunto absorvente no sentido $\mathscr{D}$-pullback) A família $\widehat{B} \in \mathscr{M}$ é dita absorvente no sentido $\mathscr{D}$ - pullback para o processo $\{U(t, \tau): t \geq \tau\}$ se para qualquer $t \in \mathbb{R}$ e qualquer $\widehat{D} \in \mathscr{D}$, existe $\tau_{0}(t, \widehat{D}) \leq t$, tal que

$$
U(t, \tau) D(\tau) \subset B(t) \text { para todo } \tau \leq \tau_{0}(t, \widehat{D})
$$

Definição 4.1.3 (Compacidade assintótica no sentido $\mathscr{D}$-pullback) O processo $\{U(t, \tau): t \geq \tau\}$ é chamado assintoticamente compacto no sentido $\mathscr{D}$-pullback, se a sequência $\left\{U\left(t, \tau_{n}\right) x_{n}\right\}$ é relativamente compacto em $X_{t}$, para qualquer $t \in \mathbb{R}, \widehat{D} \in \mathscr{D}$ e quaisquer sequências $\tau_{n}$ e $x_{n}$, com $\tau_{n} \rightarrow-\infty$, e $x_{n} \in D\left(\tau_{n}\right)$.

Teorema 4.1.4 (Existência de atrator no sentido $\mathscr{D}$-pullback) Suponhamos que o processo $\{U(t, \tau)$ : $t \geq \tau\}$ seja assintoticamente compacto no sentido $\mathscr{D}$-pullback, e que $\widehat{B} \in \mathscr{D}$ seja uma família de 
conjuntos absorventes no sentido $\mathscr{D}$-pullback para $\{U(t, \tau): t \geq \tau\}$.

Então, a família $\widehat{\mathscr{A}}=\{\mathscr{A}(t): t \in \mathbb{R}\}$ definido por $\mathscr{A}(t):=\Lambda(\widehat{B}, t)$, onde

$$
\Lambda(\widehat{D}, t):=\bigcap_{s \leq t}\left(\bar{\bigcup}_{\tau \leq s} U(t, \tau) D(\tau)^{X_{t}}\right) \text { para todo } t \in \mathbb{R}, \widehat{D} \in \mathscr{D}
$$

é um atrator no sentido $\mathscr{D}$-pullback para $\{U(t, \tau): t \geq \tau\}$, que satisfaz

$$
\mathscr{A}(t)=\varlimsup_{\widehat{D} \in \mathscr{D}} \Lambda(\widehat{D}, t) \quad X_{t} \text { para todo } t \in \mathbb{R}
$$

Além disso, $\widehat{\mathscr{A}}$ é minimal no sentido que se $\widehat{C}=\{C(t): t \in \mathbb{R}\}$ é uma família de subconjuntos fechados não vazios, tal que

$$
\lim _{\tau \rightarrow-\infty} \operatorname{dist}_{t}(U(t, \tau) B(\tau), C(t))=0 \text { para todo } t \in \mathbb{R}
$$

Então $\mathscr{A}(t) \subset C(t)$, para todo $t \in \mathbb{R}$.

O Teorema 4.1.4 é o correspondente pullback de um resultado clássico sobre existência de atratores. Encontra-se, por exemplo, em Carvalho et al [6] e Kloeden et al. [13]. Entretanto os detalhes da prova para processos com espaços $t$-dependentes $U(t, \tau): X_{\tau} \rightarrow X_{t}$ não foram apresentados. No que segue faremos a demonstração deste resultado. Para provar o Teorema 4.1.4 precisamos dos seguintes resultados.

Lema 4.1.5 Se $\widehat{B} \in \mathscr{M}$ é uma família absorvente no sentido $\mathscr{D}$-pullback, então

$$
\Lambda(\widehat{D}, t) \subset \Lambda(\widehat{B}, t) \text { para todo } \widehat{D} \in \mathscr{D}, t \in \mathbb{R}
$$

Se, além disso $\widehat{B} \in \mathscr{D}$, então

$$
\Lambda(\widehat{D}, t) \subset \Lambda(\widehat{B}, t) \subset \overline{B(t)} \text { para todo } \widehat{D} \in \mathscr{D}, t \in \mathbb{R}
$$

Demonstração: Fixemos $\widehat{D} \in \mathscr{D}, t \in \mathbb{R}$ e $y \in \Lambda(\widehat{D}, t)$. Existe uma sequência $\tau_{n} \rightarrow-\infty, \tau_{n} \leq t$, e uma sequência $x_{n} \in D\left(\tau_{n}\right)$ tal que $U\left(t, \tau_{n}\right) x_{n} \rightarrow y$. 
Como $\widehat{B}$ é uma família absorvente no sentido $\mathscr{D}$-pullback, para cada $\tau_{n}$ existe $s_{n}, s_{n} \leq \tau_{n}$, tal que $U\left(\tau_{n}, \tau\right) D(\tau) \subset B\left(\tau_{n}\right)$ para todo $\tau \leq s_{n}$.

Como $\tau \rightarrow-\infty$, dado $n \in \mathbb{N}$ existe $\left\{\tau_{n}^{1}\right\} \subset\left\{\tau_{n}\right\}$, tal que $\tau_{n}^{1} \leq s_{n} \leq \tau_{n}$, por tanto

$$
U\left(\tau_{n}, \tau_{n}^{1}\right) D\left(\tau_{n}^{1}\right) \subset B\left(\tau_{n}\right)
$$

Em particular, $U\left(\tau_{n}, \tau_{n}^{1}\right) x_{n}^{1} \in B\left(\tau_{n}\right)$, assim

$$
U\left(t, \tau_{n}^{1}\right) x_{n}^{1}=U\left(t, \tau_{n}\right) U\left(\tau_{n}, \tau_{n}^{1}\right) x_{n}^{1} \rightarrow y
$$

Como $U\left(\tau_{n}, \tau_{n}^{1}\right) x_{n}^{1} \in B\left(\tau_{n}\right)$, concluímos que $y \in \Lambda(\widehat{B}, t)$.

Finalmente, observe que se $y \in \Lambda(\widehat{B}, t)$, existe uma sequência $\tau_{n} \rightarrow-\infty, \tau_{n} \leq t$, e uma sequência $x_{n} \in B\left(\tau_{n}\right)$, tal que $U\left(t, \tau_{n}\right) x_{n} \rightarrow y$.

Mas, como $\widehat{B}$ é uma família absorvente no sentido $\mathscr{D}$-pullback e $\widehat{B} \in \mathscr{D}$, existe $\tau_{0}=\tau_{0}(\widehat{B}, t)$, tal que

$$
U(t, \tau) B(\tau) \subset B(t) \text { para todo } \tau \leq \tau_{0} .
$$

Como $\tau_{n} \rightarrow-\infty$, podemos supor $\tau_{n} \leq \tau_{0}$ para todo $n \in \mathbb{N}$, assim $U\left(t, \tau_{n}\right) x_{n} \in B(t)$ para todo $n \in \mathbb{N}$, portanto $y \in \overline{B(t)}$.

Observação 4.1.6 Como consequência direta da Proposição 4.1.5, obtemos que se $\widehat{B} \in \mathscr{D}$ é uma família absorvente no sentido $\mathscr{D}$-pullback, então

$$
\bigcup_{\widehat{D} \in \mathscr{D}} \Lambda(\widehat{D}, t) X_{t}=\Lambda(\widehat{B}, t)
$$

Lema 4.1.7 Se $\{U(t, \tau): t \geq \tau\}$ é assintoticamente compacto no sentido $\mathscr{D}$-pullback, então para $\widehat{D} \in \mathscr{D}$ e $t \in \mathbb{R}$, o conjunto $\Lambda(\widehat{D}, t)$ é não vazio, compacto e

$$
\lim _{\tau \rightarrow-\infty} \operatorname{dist}_{t}(U(t, \tau) D(\tau), \Lambda(\widehat{D}, t))=0 .
$$


Demonstração: Fixemos $\widehat{D} \in \mathscr{D}$ e $t \in \mathbb{R}$. Consideremos a sequência $\tau_{n} \rightarrow-\infty$, e a sequência $x_{n} \in D\left(\tau_{n}\right)$, então da sequência $\left\{U\left(t, \tau_{n}\right) x_{n}\right\}$ podemos extrair uma subsequência convergente

$$
U\left(t, \tau_{n_{k}}\right) x_{n_{k}} \rightarrow y
$$

Por sua construção, $y \in \Lambda(\widehat{D}, t)$, e portanto este conjunto é não vazio.

Sabemos que $\Lambda(\widehat{D}, t)$ é fechado, e conseqüentemente, a fim de provar sua compacidade é suficiente tomar qualquer sequência dada $\left\{y_{n}\right\} \subset \Lambda(\widehat{D}, t)$ e encontrar uma subsequência convergente. Primeiro, observe que como $y_{n} \in \Lambda(\widehat{D}, t)$, existe uma sequência $\tau_{n} \leq t, \tau_{n} \leq-n, \tau_{n} \rightarrow-\infty$ e uma sequência $x_{n} \in D\left(\tau_{n}\right)$ tal que $d_{t}\left(U\left(t, \tau_{n}\right) x_{n}, y_{n}\right) \leq \frac{1}{n}$. Como $\left\{U\left(t, \tau_{n}\right) x_{n}\right\}$ tem uma subsequência convergente, segue que $\left\{y_{n}\right\}$ tem uma subsequência convergente.

Finalmente, se (4.3) não é verdade, existe $\varepsilon>0$, uma sequência $\tau_{n} \rightarrow-\infty, \tau_{n} \leq t$, e uma sequência $x_{n} \in D\left(\tau_{n}\right)$, tais que

$$
d_{t}\left(U\left(t, \tau_{n}\right) x_{n}, y\right) \geq \varepsilon
$$

para todo $y \in \Lambda(\widehat{D}, t)$.

Mas a partir da sequência $\left\{U\left(t, \tau_{n}\right) x_{n}\right\}$ podemos extrair uma subsequência convergente

$$
U\left(t \tau_{n_{k}}\right) x_{n_{k}} \rightarrow y \in \Lambda(\widehat{D}, t)
$$

que contradiz (4.4).

Lema 4.1.8 Se o processo $\{U(t, \tau): t \geq \tau\}$ é assintoticamente compacto no sentido $\mathscr{D}$-pullback, então

$$
U(t, \tau) \Lambda(\widehat{D}, \tau)=\Lambda(\widehat{D}, t) \text { para todo } t \geq \tau \text { e } \widehat{D} \in \mathscr{D}
$$

Demonstração: Seja $t \geq \tau$ e $\widehat{D} \in \mathscr{D}$ fixos, e tomemos $y \in \Lambda(\widehat{D}, \tau)$. Vamos mostrar agora que 
$U(t, \tau) y \in \Lambda(\widehat{D}, t)$, e consequentemente

$$
U(t, \tau) \Lambda(\widehat{D}, \tau) \subset \Lambda(\widehat{D}, t)
$$

Nós já sabemos que existe uma sequência $s_{n} \rightarrow-\infty, s_{n} \leq \tau$, e uma sequência $x_{n} \in D\left(s_{n}\right)$ tal que $U\left(\tau, s_{n}\right) x_{n} \rightarrow y$.

Pela continuidade de $U(t, \tau)$, obtemos $U\left(t, s_{n}\right) x_{n}=U(t, \tau) U\left(\tau, s_{n}\right) x_{n} \rightarrow U(t, \tau) y$.

Portanto $U(t, \tau) y \in \Lambda(\widehat{D}, t)$.

Agora, provemos $\Lambda(\widehat{D}, t) \subset U(t, \tau) \Lambda(\widehat{D}, \tau)$. Para $y \in \Lambda(\widehat{D}, t)$ existe uma sequência $\tau_{n} \rightarrow-\infty$, $\tau_{n} \leq t$, e uma sequência $x_{n} \in D\left(\tau_{n}\right)$ tal que $U\left(t, \tau_{n}\right) x_{n} \rightarrow y$.

Como $\tau_{n} \rightarrow-\infty$, temos que existe $n_{0} \in \mathbb{N}$ tal que $\tau_{n} \leq \tau$ para todo $n \geq n_{0}$. Da sequência $\left\{U\left(\tau, \tau_{n}\right) x_{n}\right\}$ podemos extrair uma subsequência convergente (denotada da mesma maneira) para algum $z \in \Lambda(\widehat{D}, \tau)$. Segue da continuidade de $U(t, \tau)$ que $U(t, \tau) z=y$.

Portanto, $\Lambda(\widehat{D}, t)=U(t, \tau) \Lambda(\widehat{D}, \tau)$.

\section{Demonstração: (Prova do Teorema 4.1.4 - existência de atratores no sentido $\mathscr{D}$-pullback )}

A compacidade de cada $\mathscr{A}(t)$ segue do Lema 4.1.7. Além disso, pelo mesmo lema e o fato que $\bigcup_{\widehat{D} \in \mathscr{D}} \Lambda(\widehat{D}, t) \subset \mathscr{A}(t)$, obtemos

$$
\lim _{\tau \rightarrow-\infty} \operatorname{dist}_{t}(U(t, \tau) D(\tau), \mathscr{A}(t))=0 \text { para qualquer } \widehat{D} \in \mathscr{D}
$$

Como $\widehat{B} \in \mathscr{D}$, do lema 4.1.8, temos

$$
U(t, \tau) \mathscr{A}(\tau)=\mathscr{A}(t)
$$

Finalmente, seja $\widehat{C} \in \mathscr{M}$ uma família de fechados tal que

$$
\lim _{\tau \rightarrow-\infty} \operatorname{dist}_{t}(U(t, \tau) B(\tau), C(t))=0
$$


Seja $y \in \mathscr{A}(t)$, existe uma sequência $\tau_{n} \rightarrow-\infty, \tau_{n} \leq t$, e uma sequência $x_{n} \in B\left(\tau_{n}\right)$, tal que $U(t, \tau) x_{n} \rightarrow y$.

De (4.6), seque que $U\left(t, \tau_{n}\right) x_{n} \in C(t)$ para $n \geq n_{0}$, assim $y \in \overline{C(t)}=C(t)$.

\subsection{Resultado principal}

Nesta Seção apresentamos a nossa principal contribuição. Vamos considerar agora as seguintes hipóteses:

- H1-H2-H3 dadas no Capítulo 2;

- (H4) $\mathscr{O}$ é convexo;

- (H5) Assumos também que as seguintes hipóteses de dissipação sejam satisfeitas

$$
\begin{gathered}
\exists k \geq 1, C>0, \mu_{0}>0, \forall \mu \in\left(0, \mu_{0}\right], \exists C_{\mu} \in \mathbb{R}, \\
k G(s)-\mu s^{2}-C_{\mu} \leq s g(s) \leq C s^{2} \forall s \in \mathbb{R}, \\
\exists v_{0}>0, \forall v \in\left(0, v_{0}\right], \exists C_{v} \in \mathbb{R} \\
-v s^{2}-C_{v} \leq G(s) \forall s \in \mathbb{R},
\end{gathered}
$$

- (H6) Com relação à função $f$,

$$
\int_{-\infty}^{t} e^{\sigma \theta}\|f(\theta)\|_{H^{-1}\left(\mathscr{O}_{\theta}\right)}^{2} d \theta<\infty \text { para todo } t \in \mathbb{R}
$$

onde $\sigma$ é uma constante positiva que será caracterizada posteriormente.

A partir de agora trabalharemos com uma base de atração fixa. Seja $R_{\sigma}$ o conjunto de todas as funções $\rho: \mathbb{R} \longrightarrow[0, \infty)$ tal que 


$$
e^{\sigma \tau} \rho^{2}(\tau) \rightarrow 0 \text { quando } \tau \rightarrow-\infty
$$

e $\mathscr{D}_{\sigma}$ a base de atração formada pelas clases de todas as famílias

$$
\begin{gathered}
\widehat{D}:=\left\{D(t): t \in \mathbb{R}, D(t) \subset X_{t}, D(t) \neq \emptyset\right\} \text { tal que } \\
D(t) \subset\left\{\left(u, u^{\prime}\right) \in X_{t}:\left\|\left(u, u^{\prime}\right)\right\|_{X_{t}} \leq \rho_{\widehat{D}}(t)\right\} \text { para algum } \rho_{\widehat{D}} \in R_{\sigma} .
\end{gathered}
$$

Teorema 4.2.1 Suponha que $f \in H_{\text {loc }}^{1}\left(\mathbb{R}, L^{2}\left(\mathscr{O}_{t}\right)\right)$ com condições $(H 1)-(H 6)$ satisfeitas, tal que $0<\frac{\sigma(N-1)}{N-2}<\frac{\eta}{5}$, para algum $\sigma>0$. Então o processo $\{U(t, \tau): t \geq \tau\}$ associado ao Problema 3.1 possui um atrator no sentido $\mathscr{D}_{\sigma}-$ pullback.

Observação 4.2.2 Observemos que para provar que o processo de evolução $\{U(t, \tau): t \geq \tau\}$ associado ao problema (3.1) tem um atrator no sentido DD-pullback é necessário verificar às hipóteses do Teorema 4.1.4

A seguir, nas seções seguintes verificamos às hipóteses do Teorema 4.1.4.

\subsection{Existência de um conjunto absorvente $\mathscr{D}_{\sigma}$-pullback}

Nesta seção mostraremos a existência de conjuntos absorventes no sentido $\mathscr{D}$-pullback para o problema de onda semilinear (3.1) em espaços de Hilbert $X_{t}=H_{0}^{1}\left(\mathscr{O}_{t}\right) \times L^{2}\left(\mathscr{O}_{t}\right)$.

Observação 4.3.1 Seja $\lambda_{1, t}$ o primeiro autovalor de $-\Delta$ em $H_{0}^{1}\left(\Omega_{t}\right)$, com $\Omega_{t}:=\bigcup_{s \leq t} \mathscr{O}_{s}$ limitado para todo $t \in \mathbb{R}$. Dado qualquer $t \in \mathbb{R}$, pela convexidade de $\mathscr{O}$ e a hipótese $(\boldsymbol{H 1})$, obtemos

$$
h_{0} \mathscr{O}:=\left\{x=h_{0} y ; y \in \mathscr{O}\right\} \subset \Omega_{t} \subset\left\{x=h_{1} y ; y \in \mathscr{O}\right\}:=h_{1} \mathscr{O} .
$$


Assim, se denotamos como $\lambda_{0}$ e $\lambda_{1}$ ao primeiro autovalor de $-\Delta$ sobre $H_{0}^{1}\left(h_{0} \mathscr{O}\right)$ e $H_{0}^{1}\left(h_{1} \mathscr{O}\right)$ respectivamente, resulta das propriedades dos autovalores, que

$$
\lambda_{1} \leq \lambda_{1, t} \leq \lambda_{0} \text { para todo } t \in \mathbb{R}
$$

A existência de um conjunto absorvente em $X_{t}$ é assegurada pelo seguinte lema.

Lema 4.3.2 Considere $f \in H_{\text {loc }}^{1}\left(\mathbb{R}, L^{2}\left(\mathscr{O}_{t}\right)\right)$ e suponha que $0<\frac{\sigma(N-1)}{N-2}<\frac{\eta}{5}$ para algum $\sigma>0$. Então a família de conjuntos

$$
\widehat{B}:=\{B(t): t \in \mathbb{R}\}=\left\{\left\{\left(u, u^{\prime}\right) \in X_{t}:\left\|\left(u, u^{\prime}\right)\right\|_{X_{t}} \leq R(t)\right\}: t \in \mathbb{R}\right\}
$$

onde

$$
R^{2}(t)=C_{1} e^{-\sigma t} \int_{-\infty}^{t} e^{\sigma \theta}\|f(\theta)\|_{H^{-1}\left(\mathscr{O}_{\theta}\right)}^{2} d \theta+C_{2}
$$

é uma família de conjuntos absorvente no sentido $\mathscr{D}_{\sigma}$-pullback para o processo de evolução $\{U(t, \tau)$ : $t \geq \tau\}$

Além disso, $\widehat{B} \in \mathscr{D}_{\sigma}$.

Demonstração: Fixemos um conjunto arbitrário $\widehat{D} \in \mathscr{D}_{\sigma}$ e consideremos as soluções do problema (3.1) dadas por $\left(u(t), u^{\prime}(t)\right)=U(t, \tau)\left(u_{\tau 0}, u_{\tau 1}\right) \operatorname{com}\left(u_{\tau 0}, u_{\tau 1}\right) \in D(\tau)$. Escolhemos $\delta>0$, satisfazendo $\frac{\sigma(N-1)}{N-2}<\delta<\frac{\eta-\delta}{4}$ para todo $t \in \mathbb{R}$.

A energia modificada do problema é dada por

$$
E(t)=\frac{1}{2}\left|u^{\prime}(t)+\delta u(t)\right|_{t}^{2}+\frac{1}{2} \delta(\eta-\delta)|u(t)|_{t}^{2}+\frac{1}{2}\|u\|_{t}^{2}+\int_{\mathscr{O}_{t}} G(u(t)) d x
$$


Multiplicando $(3.1)_{1}$ por $u^{\prime}(\theta)+\delta u(\theta)$ e integrando sobre $\mathscr{O}_{\theta}$ obtemos a seguinte igualdade

$$
\begin{aligned}
\frac{d}{d \theta} E(\theta)- & \frac{h^{\prime}}{h} \int_{\partial \mathscr{O}_{\theta}} E(\theta)(x \cdot \bar{v}) d\left(\partial \mathscr{O}_{\theta}\right)+(\eta-\delta)\left|u^{\prime}(\theta)\right|_{\theta}^{2} \\
& +\delta \|\left. u(\theta)\right|_{\theta} ^{2}+\delta \int_{\mathscr{O}_{\theta}} u(\theta) g(u(\theta)) d x=\left(f(\theta), u^{\prime}(\theta)+\delta u(\theta)\right)_{\theta}
\end{aligned}
$$

Assim,

$$
\frac{d}{d \theta} E(\theta)+(\eta-\delta)\left|u^{\prime}(\theta)\right|_{\theta}^{2}+\delta\|u(\theta)\|_{\theta}^{2}+\delta \int_{\mathscr{O}_{\theta}} u(\theta) g(u(\theta)) d x \leq\left(f(\theta), u^{\prime}(\theta)+\delta u(\theta)\right)_{\theta}
$$

Escolhemos $\mu_{0}=2 \delta(\eta-\delta)$ em (4.7) e (4.8), utilizando as desigualdades de Hölder e de Young, obtemos

$$
\frac{d}{d \theta} E(\theta)+\delta E(\theta) \leq \frac{1}{\eta-\delta}|| f(\theta) \|_{H^{-1}\left(\mathscr{O}_{\theta}\right)}^{2}+\delta h_{1}^{N}\left(C_{\mu_{0}}+(k-1) C_{V}\right)|\mathscr{O}|
$$

Note que

$$
\frac{d}{d \theta}\left(e^{\sigma \theta} E(\theta)\right)=\sigma e^{\sigma \theta} E(\theta)+e^{\sigma \theta} \frac{d}{d \theta} E(\theta)
$$

De (4.15) e (4.16), sendo $\sigma<\delta$, temos

$$
\frac{d}{d \theta}\left(e^{\sigma \theta} E(\theta)\right) \leq \frac{e^{\sigma \theta}}{\eta-\delta}|| f(\theta) \|_{H^{-1}\left(\mathscr{O}_{\theta}\right)}^{2}+e^{\sigma \theta} \delta h_{1}^{N}\left(C_{\mu_{0}}+(k-1) C_{V}\right)|\mathscr{O}|
$$

Assim, integrando de $\tau$ até $t$, resulta

$$
E(t) \leq e^{-\sigma(t-\tau)} E(\tau)+\frac{e^{-\sigma t}}{\eta-\delta} \int_{\tau}^{t} e^{\sigma \theta}\|f(\theta)\|_{H^{-1}\left(\mathscr{O}_{\theta}\right)}^{2} d \theta+C e^{-\sigma t} \int_{\tau}^{t} e^{\sigma \theta} d \theta
$$

onde $C=\delta h_{1}^{N}\left(C_{\mu_{0}}+(k-1) C_{V}\right)|\mathscr{O}|$.

Nosso seguinte passo é estimar $E(t)$ e $E(\tau)$. 


\section{Estimando E(t)}

Por (H.3), (H5) e os teoremas de imersão, temos

$$
E(t) \geq \frac{1}{2}\left|u^{\prime}(t)+\delta u(t)\right|_{t}^{2}+\frac{1}{2}|| u(t) \|_{t}^{2}-C_{v}\left|\mathscr{O}_{t}\right|
$$

e note que $\left\|u(t)||_{t}^{2}+\left|u^{\prime}(t)\right|_{t}^{2} \leq\left(1+2 \lambda_{1, t}^{-1} \delta^{2}\right)\right\| u(t) \|_{t}^{2}+2\left|u^{\prime}(t)+\delta u(t)\right|_{t}^{2}$. De (4.10) obtemos que $\lambda_{1, t}^{-1} \leq \lambda_{1}^{-1}$, portanto

$$
\left\|\left.u(t)\right|_{t} ^{2}+\left|u^{\prime}(t)\right|_{t}^{2} \leq\left(1+2 \lambda_{1}^{-1} \delta^{2}\right)\right\| u(t) \|_{t}^{2}+2\left|u^{\prime}(t)+\delta u(t)\right|_{t}^{2}
$$

Observemos que

$$
\left(1+2 \lambda_{1}^{-1} \delta^{2}\right)\|u(t)\|_{t}^{2}+2\left|u^{\prime}(t)+\delta u(t)\right|_{t}^{2} \leq \max \left\{4,2+4 \lambda_{1}^{-1} \delta^{2}\right\}\left(\frac{1}{2}\|u(t)\|_{t}^{2}+\frac{1}{2}\left|u^{\prime}(t)+\delta u(t)\right|_{t}^{2}\right) .
$$

Finalmente de (4.19), (4.20) e (4.21) resulta que

$$
\frac{1}{v_{1}}\left(|| u(t)||_{t}^{2}+\left|u^{\prime}(t)\right|_{t}^{2}\right)-C_{v}\left|\mathscr{O}_{t}\right| \leq E(t)
$$

onde $v_{1}=\max \left\{4,2+4 \lambda_{1}^{-1} \delta^{2}\right\}$.

\section{Estimando $\boldsymbol{E}(\tau)$}

Escolhendo $\mu_{0}>0$ apropriado, obtemos

$$
\begin{aligned}
E(\tau) & \leq\left|u_{\tau 1}\right|_{\tau}^{2}+\delta^{2}\left|u_{\tau 0}\right|_{\tau}^{2}+\frac{1}{2} \delta(\eta-\delta)\left|u_{\tau 0}\right|_{\tau}^{2}+\frac{1}{2}\left\|u_{\tau 0}\right\|_{\tau}^{2}+\frac{1}{k} \int_{\mathscr{O}_{\tau}}\left(\left(\mu_{0}+C\right)\left|u_{\tau 0}\right|^{2}+C_{\mu_{0}}\right) d x \\
& =\left|u_{\tau 1}\right|_{\tau}^{2}+\left[\delta^{2}+\frac{1}{2} \delta(\eta-\delta)+\frac{\mu_{0}+C}{k}\right]\left|u_{\tau 0}\right|_{\tau}^{2}+\frac{1}{2}\left\|u_{\tau 0}\right\|_{\tau}^{2}+\frac{C_{\mu_{0}} h_{1}^{N}}{k}|\mathscr{O}| .
\end{aligned}
$$

Da desigualdade de Poincaré, existe uma constante $C^{\prime}$, tal que

$$
\begin{aligned}
E(\tau) & \leq\left|u_{\tau 1}\right|_{\tau}^{2}+\left\{\delta^{2}+\frac{1}{2} \delta(\eta-\delta)+\frac{\mu_{0}+C}{k}\right\} C^{\prime}\left\|u_{\tau 0}\right\|_{\tau}^{2}+\frac{1}{2}\left\|u_{\tau 0}\right\|_{\tau}^{2}+\frac{C_{\mu_{0}} h_{1}^{N}}{k}|\mathscr{O}| \\
& =\left|u_{\tau 1}\right|_{\tau}^{2}+\left\{\frac{1}{2}+\delta^{2}+\frac{1}{2} \delta(\eta-\delta)+\frac{\mu_{0}+C}{k}\right\} C^{\prime}\left\|u_{\tau 0}\right\|_{\tau}^{2}+\frac{C_{\mu_{0}} h_{1}^{N}}{k}|\mathscr{O}| .
\end{aligned}
$$


Assim

$$
E(\tau) \leq v_{2}\left(\left|u_{\tau 1}\right|_{\tau}^{2}+\left\|u_{\tau 0}\right\|_{\tau}^{2}+1\right)
$$

onde $v_{2}=\max \left\{1,\left\{\frac{1}{2}+\delta^{2}+\frac{1}{2} \delta(\eta-\delta)+\frac{\mu_{0}+C}{k}\right\} C^{\prime}, \quad \frac{\left(C+C_{\mu_{0}}\right) h_{1}^{N}}{k}|\mathscr{O}|\right\}$.

Portanto, substituindo (4.22) e (4.23) em (4.18), temos

$$
\begin{aligned}
\|u(t)\|_{t}^{2}+\left|u^{\prime}(t)\right|_{t}^{2} \leq & v_{1} v_{2} e^{-\sigma(t-\tau)}\left(\left|u_{\tau 1}\right|_{\tau}^{2}+\left\|u_{\tau 0}\right\|_{\tau}^{2}+1\right)+\frac{v_{1} e^{-\sigma t}}{\eta-\delta} \int_{\tau}^{t} e^{\sigma \theta}\|f(\theta)\|_{H^{-1}\left(\mathscr{O}_{\theta}\right)}^{2} d \theta \\
& +v_{1} C e^{-\sigma t} \int_{\tau}^{t} e^{\sigma \theta} d \theta+v_{1} C_{v} h_{1}^{N}|\mathscr{O}|
\end{aligned}
$$

Vamos estimar o lado direito de (4.24).

- $v_{1} C e^{-\sigma t} \int_{\tau}^{t} e^{\sigma \theta} d \theta=v_{1} C \frac{1}{\sigma}-\frac{v_{1} C}{\sigma} e^{-\sigma(t-\tau)} \leq v_{1} C \sigma^{-1}$.

Substituindo a estimativa anterior em (4.24), resulta

$$
\begin{aligned}
\|u(t)\|_{t}^{2}+\left|u^{\prime}(t)\right|_{t}^{2} & \leq C_{1} e^{-\sigma(t-\tau)}\left(\left|u_{\tau 1}\right|_{\tau}^{2}+\left\|u_{\tau 0}\right\|_{\tau}^{2}+1\right) \\
& +C_{2} e^{-\sigma t} \int_{\tau}^{t} e^{\sigma \theta}\|f(\theta)\|_{H^{-1}\left(\mathscr{O}_{\theta}\right)}^{2} d \theta+C_{3}
\end{aligned}
$$

onde $C_{1}=v_{1} v_{2}, C_{2}=v_{1}(\eta-\delta)^{-1}$ e $C_{3}=v_{1}\left(C_{1} \lambda_{1}^{-1}+C_{v} h_{1}^{N}|\mathscr{O}|\right)$.

Portanto

$$
\begin{aligned}
\|u(t)\|_{t}^{2}+\left|u^{\prime}(t)\right|_{t}^{2} \leq & C_{1} e^{-\sigma t} e^{\sigma \tau}\left(\left|u_{\tau 1}\right|_{\tau}^{2}+\left\|u_{\tau 0}\right\|_{\tau}^{2}+1\right) \\
& +C_{2} e^{-\sigma t} \int_{\tau}^{t} e^{\sigma \theta}\|f(\theta)\|_{H^{-1}\left(\mathscr{O}_{\theta}\right)}^{2} d \theta+C_{3} .
\end{aligned}
$$

Como $\left(u_{\tau 0}, u_{\tau 1}\right) \in D(\tau)$ existe $\rho_{\widehat{D}} \in R_{\sigma}$, tal que

$$
\left\|\left(u_{\tau 0}, u_{\tau 1}\right)\right\|_{X_{\tau}}:=\left(\left\|u_{\tau 0}\right\|_{\tau}^{2}+\left|u_{\tau 1}\right|_{\tau}^{2}\right)^{\frac{1}{2}} \leq \rho_{\widehat{D}}(\tau) .
$$


Reeplazando (4.27) em (4.26), obtemos

$$
\begin{aligned}
\left\|\left(u(t), u^{\prime}(t)\right)\right\|_{X_{t}}^{2}:=\|u(t)\|_{t}^{2}+\left|u^{\prime}(t)\right|_{t}^{2} \leq & C_{1} e^{-\sigma t} e^{\sigma \tau}\left(\rho_{\widehat{D}}^{2}(\tau)+1\right) \\
& +C_{2} e^{-\sigma t} \int_{\tau}^{t} e^{\sigma \theta}\|f(\theta)\|_{H^{-1}\left(\mathscr{O}_{\theta}\right)}^{2} d \theta+C_{3} .
\end{aligned}
$$

Lembremos que nossa base de atração foi escolhida de modo que

$$
e^{\sigma \tau} \rho_{\widehat{D}}^{2}(\tau) \rightarrow 0 \text { quando } \tau \rightarrow-\infty
$$

Portanto, escrevendo $R^{2}(t)=C_{1} e^{-\sigma t} \int_{-\infty}^{t} e^{\sigma \theta}\|f(\theta)\|_{H^{-1}\left(\mathscr{O}_{\theta}\right)}^{2} d \theta+C_{2}$ com $C_{1}=C_{2}$ e $C_{2}=C_{3}$, segue que $\widehat{B}=\left\{\left\{\left(u, u^{\prime}\right) \in X_{t}:\left\|\left(u, u^{\prime}\right)\right\|_{X_{t}} \leq R(t)\right\}: t \in \mathbb{R}\right\}$ é uma família de conjuntos absorventes no sentido $\mathscr{D}_{\sigma}$-pullback para o processo de evolução associado ao problema de onda semilinear.

Para ver que $\widehat{B}$ pertence a $\mathscr{D} \sigma$ precisamos provar que $e^{\sigma t} R^{2}(t) \rightarrow 0$, quando $t \rightarrow-\infty$. Com efeito, para $t \in \mathbb{R}$, temos

$$
\begin{aligned}
e^{\sigma t} R^{2}(t) & =e^{\sigma t} C_{1} e^{-\sigma t} \int_{-\infty}^{t} e^{\sigma \theta}\|f(\theta)\|_{H^{-1}\left(\mathscr{O}_{\theta}\right)}^{2} d \theta+e^{\sigma t} C_{2} \\
& =C_{1} \int_{-\infty}^{t} e^{\sigma \theta}\|f(\theta)\|_{H^{-1}\left(\mathscr{O}_{\theta}\right)}^{2} d \theta+e^{\sigma t} C_{2}
\end{aligned}
$$

Portanto

$$
e^{\sigma t} R^{2}(t)=C_{1} \int_{-\infty}^{t} e^{\sigma \theta}\|f(\theta)\|_{H^{-1}\left(\mathscr{O}_{\theta}\right)}^{2} d \theta+e^{\sigma t} C_{2} \rightarrow 0 \text { quando } t \rightarrow-\infty
$$

Assim fica provado a existência de uma família absorvente $\mathscr{D}_{\sigma}$-pullback.

\subsection{Compacidade assintótica $\mathscr{D}_{\sigma}$-pullback}

Prosseguindo, mostraremos que o processo $\{U(t, \tau): t \geq \tau\}$ associado ao problema de onda semilinear possui a propriedade de compacidade assintótica no sentido $\mathscr{D} \sigma$-Pullback. 
Definição 4.4.1 Seja X um espaço de Banach e B um subconjunto limitado de X. Uma função $\Phi(.,$.$) definido sobre X \times X$ é uma função contractiva sobre $B \times B$, se para qualquer sequência $\left\{x_{n}\right\}_{n \in \mathbb{N}} \subset B$, existe uma subsequência $\left\{x_{n_{k}}\right\}_{k \in \mathbb{N}} \subset\left\{x_{n}\right\}_{n \in \mathbb{N}}$ tal que

$$
\lim _{k \rightarrow \infty} \lim _{l \rightarrow \infty} \Phi\left(x_{n_{k}}, x_{n_{l}}\right)=0
$$

O teorema a seguir é uma adaptação para problemas não autônomos em domínios não cilíndricos apresentados em [20, Teorema 4.2] e [22, Teorema 2.6].

Teorema 4.4.2 Suponhamos que a família de conjuntos

$$
\widehat{B}=\left\{\left\{\left(u, u^{\prime}\right) \in X_{t}:\left\|\left(u, u^{\prime}\right)\right\|_{X_{t}} \leq R(t)\right\}: t \in \mathbb{R}\right\}
$$

satisfaz que para qualquer $\widehat{D}=\{D(\tau): \tau \in \mathbb{R}\} \in \mathscr{D}_{\sigma}$, e qualquer $t \in \mathbb{R}$, existe $\tau_{0}(t, \widehat{D}) \leq t$, satisfazendo

$$
U(t, \tau) D(\tau) \subset\left\{\left(u, u^{\prime}\right) \in X_{t}:\left\|\left(u, u^{\prime}\right)\right\|_{X_{t}} \leq R(t)\right\} \text { para todo } \tau \leq \tau_{0}(t, \widehat{\widehat{D}}) .
$$

Suponhamos que para qualquer $\varepsilon>0$ e qualquer $t \in \mathbb{R}$, existe $\tau_{0}(t, \widehat{B})$ e uma função contractiva $\Phi_{\tau_{0}}(.,$.$) definda sobre \widehat{B}=\{B(\tau): \tau \in \mathbb{R}\}$ tal que

$$
\|U(t, \tau) x-U(t, \tau) y\|_{X_{t}} \leq \varepsilon+\Phi_{\tau_{0}}(x, y)
$$

para todo $x, y \in B(\tau), \tau \leq \tau_{0}$.

Então $\{U(t, \tau): t \geq \tau\}$ é assintoticamente compacto no sentido $\mathscr{D}_{\sigma}$-pullback.

Demonstração: Fixemos $t \in \mathbb{R}$. Consideremos $\tau_{n} \rightarrow-\infty, \widehat{D} \in \mathscr{D}_{\sigma}$ e $x_{n} \in D\left(\tau_{n}\right)$. Seja $\varepsilon_{m}>0$, tal que $\varepsilon_{m} \rightarrow 0$.

Para $\varepsilon_{1}$, existe $\tau_{1} \leq t$, e uma função contractiva $\Phi_{\tau_{1}}$ definida sobre $B(\tau) \times B(\tau)$ tal que

$$
\|U(t, \tau) x-U(t, \tau) y\|_{X_{t}} \leq \varepsilon_{1}+\Phi_{\tau_{1}}(x, y) \text { para todo } x, y \in B(\tau) \text { com } \tau \leq \tau_{1} .
$$


Como $\tau_{n} \rightarrow-\infty$, podemos assumir que $\tau_{n} \leq \tau \leq \tau_{1}$, e por (4.30), temos

$$
U\left(\tau, \tau_{n}\right) D\left(\tau_{n}\right) \subset B(\tau) \text { para todo } \tau_{n} \leq \tau
$$

Assim de (4.32), obtemos

$$
y_{n}=U\left(\tau, \tau_{n}\right) x_{n} \in B(\tau) \text { para todo } n \in \mathbb{N} \text {. }
$$

Assim de (4.31) e (4.33), temos

$$
\left\|U(t, \tau) y_{n}-U(t, \tau) y_{m}\right\|_{X_{t}} \leq \varepsilon_{1}+\Phi_{\tau_{1}}\left(y_{n}, y_{m}\right)
$$

para todo $n, m \in \mathbb{N}$. Da definição (4.4.1), podemos obter uma subsequência $\left(y_{n}^{1}\right)_{n \in \mathbb{N}}$ de $\left(y_{n}\right)_{n \in \mathbb{N}}$ tal que

$$
\lim _{l \rightarrow \infty} \Phi_{\tau_{1}}\left(y_{k}^{1}, y_{l}^{1}\right) \leq \frac{\varepsilon_{1}}{2} \text { para todo } k \in \mathbb{N},
$$

então por (4.34) e (4.35), temos

$$
\begin{aligned}
& \limsup _{k \rightarrow \infty} \sup _{p \in \mathbb{N}}\left\|U\left(t, \tau_{k+p}^{1}\right) x_{k+p}^{1}-U\left(t, \tau_{k}^{1}\right) x_{k}^{1}\right\|_{X_{t}} \\
& \leq \limsup _{k \rightarrow \infty} \sup _{p \in \mathbb{N}} \limsup _{l \rightarrow \infty}\left\|U\left(t, \tau_{k+p}^{1}\right) x_{k+p}^{1}-U\left(t, \tau_{l}^{1}\right) x_{l}^{1}\right\|_{X_{t}} \\
& +\limsup \limsup \left\|U\left(t, \tau_{k}^{1}\right) x_{k}^{1}-U\left(t, \tau_{l}^{1}\right) x_{l}^{1}\right\|_{X_{t}} \\
& \leq \varepsilon_{1}+\limsup _{k \rightarrow \infty} \sup _{p \in \mathbb{N}} \lim _{l \rightarrow \infty} \Phi_{\tau_{1}}\left(y_{k+p}^{1}, y_{l}^{1}\right)+\varepsilon_{1}+\lim _{k \rightarrow \infty} \lim _{l \rightarrow \infty} \Phi_{\tau_{1}}\left(y_{k}^{1}, y_{l}^{1}\right) \\
& \leq 4 \varepsilon_{1} \text {. }
\end{aligned}
$$

Portanto, existe $N_{1} \in \mathbb{N}$, tal que

$$
\left\|U\left(t, \tau_{k}^{1}\right) x_{k}^{1}-U\left(t, \tau_{l}^{1}\right) x_{l}^{1}\right\|_{X_{t}} \leq 5 \varepsilon_{1} \text { para todo } k, l \geq N_{1}
$$


Para $\varepsilon_{2}>0$, existe $\tau_{2} \leq t$, tal que

$$
\|U(t, \tau) x-U(t, \tau) y\|_{X_{t}} \leq \varepsilon_{2}+\Phi_{\tau_{2}}(x, y) \text { para todo } x, y \in B(\tau) \text { com } \tau \leq \tau_{2} \text {. }
$$

Como $\tau_{n}^{1} \rightarrow-\infty$, podemos escolher $\left(\tau_{n}^{(2)}\right) \subset\left(\tau_{n}^{1}\right)$, tal que $\tau_{n}^{(2)} \leq \tau \leq \tau_{2}$.

Por (4.30), temos

$$
U\left(\tau, \tau_{n}^{(2)}\right) D\left(\tau_{n}^{(2)}\right) \subset B(\tau) \text { para todo } n \in \mathbb{N} \text {. }
$$

Segue de (4.39), que

$$
y_{n}^{(2)}=U\left(\tau, \tau_{n}^{(2)}\right) x_{n}^{(2)} \in B(\tau) \text { para todo } n \in \mathbb{N} .
$$

Portanto, de (4.38) e (4.40), temos

$$
\left\|U(t, \tau) y_{n}^{(2)}-U(t, \tau) y_{m}^{(2)}\right\|_{X_{t}} \leq \varepsilon_{2}+\Phi_{\tau_{2}}\left(y_{n}^{(2)}, y_{m}^{(2)}\right)
$$

para todo $n, m \in \mathbb{N}$. Da definição (4.4.1), podemos obter uma subsequência (denotada da mesma maneira), tal que

$$
\lim _{l \rightarrow \infty} \Phi_{\tau_{2}}\left(y_{k}^{(2)}, y_{l}^{(2)}\right) \leq \frac{\varepsilon_{2}}{2} \text { para todo } k \in \mathbb{N}
$$

e então por (4.41) e (4.42), temos

$$
\begin{aligned}
\limsup _{k \rightarrow \infty} \sup _{p \in \mathbb{N}}\left\|U\left(t, \tau_{k+p}^{(2)}\right) x_{k+p}^{(2)}-U\left(t, \tau_{k}^{1}\right) x_{k}^{(2)}\right\| X_{t} \\
\leq \quad \limsup \sup _{k \rightarrow \infty} \limsup _{l \rightarrow \infty}\left\|U\left(t, \tau_{k+p}^{(2)}\right) x_{k+p}^{(2)}-U\left(t, \tau_{l}^{(2)}\right) x_{l}^{(2)}\right\|_{X_{t}} \\
\quad+\limsup _{k \rightarrow \infty} \limsup _{l \rightarrow \infty}\left\|U\left(t, \tau_{k}^{(2)}\right) x_{k}^{(2)}-U\left(t, \tau_{l}^{(2)}\right) x_{l}^{(2)}\right\|_{X_{t}} \\
\leq \varepsilon_{2}+\limsup _{k \rightarrow \infty} \sup _{p \in \mathbb{N}} \lim _{l \rightarrow \infty} \Phi_{\tau_{2}}\left(y_{k+p}^{(2)}, y_{l}^{(2)}\right)+\varepsilon_{2}+\lim _{k \rightarrow \infty} \lim _{l \rightarrow \infty} \Phi_{\tau_{2}}\left(y_{k}^{(2)}, y_{l}^{(2)}\right) \\
\leq 4 \varepsilon_{2} .
\end{aligned}
$$


Portanto, existe $N_{2} \geq N_{1}$, tal que

$$
\left\|U\left(t, \tau_{k}^{(2)}\right) x_{k}^{(2)}-U\left(t, \tau_{l}^{(2)}\right) x_{l}^{(2)}\right\|_{X_{t}} \leq 5 \varepsilon_{2} \text { para todo } k, l \geq N_{2}
$$

Por indução, para cada $m \geq 1$, existe uma subsequência $\left\{U\left(t, \tau_{k}^{(m+1)}\right) x_{k}^{(m+1)}\right\}_{k \in \mathbb{N}} \operatorname{de}\left\{U\left(t, \tau_{k}^{(m)}\right) x_{k}^{(m)}\right\}_{k \in \mathbb{N}}$ e algum $N_{m+1}$ tal que

$$
\left\|U\left(t, \tau_{k}^{(m+1)}\right) x_{k}^{(m+1)}-U\left(t, \tau_{l}^{(m+1)}\right) x_{l}^{(m+1)}\right\|_{X_{t}} \leq \varepsilon_{m+1} \text { para todo } k, l \geq N_{m+1}
$$

Agora, consideramos a subsequência diagonal $\left\{U\left(t, \tau_{k}^{(k)}\right) x_{k}^{(k)}\right\}_{k \in \mathbb{N}}$. Como para cada $m \in \mathbb{N}$, a sequência $\left\{U\left(t, \tau_{k}^{(k)}\right) x_{k}^{(k)}\right\}_{k \geq m}$ é uma subsequência de $\left\{U\left(t, \tau_{k}^{(k)}\right) x_{k}^{(k)}\right\}_{k \in \mathbb{N}}$, temos assim

$$
\left\|U\left(t, \tau_{k}^{(k)}\right) x_{k}^{(k)}-U\left(t, \tau_{l}^{(l)}\right) x_{l}^{(l)}\right\|_{X_{t}} \leq 5 \varepsilon_{m} \text { para todo } k, l \geq \max \left\{m, N_{m}\right\}
$$

e então, como $\varepsilon_{m} \rightarrow 0$ quando $m \rightarrow \infty$, concluímos que $\left\{U\left(t, \tau_{k}^{(k)}\right) x_{k}^{(k)}\right\}_{k \in \mathbb{N}}$ é uma sequência de Cauchy em $X_{t}$.

Agora no seguinte teorema demostraremos que o processo $\{U(t, \tau): t \geq \tau\}$ é assintoticamente compacto no sentido $\mathscr{D}_{\sigma}$-pullback.

Teorema 4.4.3 Com as hipóteses do teorema (3.1.2) e suponhamos que $0<\frac{\sigma(N-1)}{N-2}<\frac{\eta}{5}$. Então o processo de evolução $\{U(t, \tau): t \geq \tau\}$ gerado pelas soluções fracas do problema (2.1) é assintoticamente compacto no sentido $\mathscr{D} \sigma$-pullback.

\section{Demonstração: [Teorema 4.4.3]}

Fixemos $t \in \mathbb{R}$. Seja $\left(u_{i}(\theta), u_{i}^{\prime}(\theta)\right)=U(\theta, \tau)\left(u_{0 \tau}^{i}, u_{1 \tau}^{i}\right)$ as correspondentes soluções fracas do problema (2.1), associado as condições iniciais $\left(u_{0 \tau}^{i}, u_{1 \tau}^{i}\right) \in \widehat{D}$, com $\widehat{D} \in \mathscr{D}_{\sigma}$, para $i=1,2$.

Denotemos por $w(\theta)=u_{1}(\theta)-u_{2}(\theta)$ a diferença de soluções fracas, então $w$ satisfaz

$$
w^{\prime \prime}(\theta)+\eta w^{\prime}(\theta)-\Delta w(\theta)=g\left(u_{1}(\theta)\right)-g\left(u_{2}(\theta)\right),
$$


com condições iniciais $\left(w(\tau), w^{\prime}(\tau)\right)=\left(u_{0 \tau}^{1}, u_{1 \tau}^{1}\right)-\left(u_{0 \tau}^{2}, u_{1 \tau}^{2}\right)$.

Definamos $E_{u}(\theta)=\frac{1}{2}\left(|\nabla u(\theta)|_{\theta}^{2}+\left|u^{\prime}(\theta)\right|_{\theta}^{2}\right)$.

Multiplicando (4.47) por $e^{\sigma \theta} w^{\prime}(\theta)$ e integrando sobre $\mathscr{O}_{\theta}$, obtemos

$$
\begin{aligned}
\frac{d}{d \theta}\left(e^{\sigma \theta} E_{w}(\theta)\right)+\eta e^{\sigma \theta}\left|w^{\prime}(\theta)\right|_{\theta}^{2} \leq & \sigma e^{\sigma \theta} E_{w}(\theta) \\
& +e^{\sigma \theta}\left(g\left(u_{2}\right)-g\left(u_{1}\right), w^{\prime}(\theta)\right)_{\theta} .
\end{aligned}
$$

Integrando (4.48) de $s$ até $t$, obtemos

$$
\begin{aligned}
& e^{\sigma t} E_{w}(t)-e^{\sigma s} E_{w}(s)+\eta \int_{s}^{t} e^{\sigma \theta}\left|w^{\prime}(\theta)\right|_{\theta}^{2} d \theta \\
& \quad \leq \sigma \int_{s}^{t} e^{\sigma \theta} E_{w}(\theta) d \theta+\int_{s}^{t} e^{\sigma \theta}\left(g\left(u_{2}\right)-g\left(u_{1}\right), w^{\prime}(\theta)\right)_{\theta} d \theta .
\end{aligned}
$$

Logo, integrando (4.49) entre $\tau$ e $t$ com respecto a $s$, temos

$$
\begin{aligned}
& (t-\tau) e^{\sigma t} E_{w}(t)-\int_{\tau}^{t} e^{\sigma s} E_{w}(s) d s+\eta \int_{\tau}^{t} \int_{s}^{t} e^{\sigma \theta}\left|w^{\prime}(\theta)\right|_{\theta}^{2} d \theta d s \\
& \leq \sigma \int_{\tau}^{t} \int_{s}^{t} e^{\sigma \theta} E_{w}(\theta) d \theta d s+\int_{\tau}^{t} \int_{s}^{t} e^{\sigma \theta}\left(g\left(u_{2}\right)-g\left(u_{1}\right), w^{\prime}(\theta)\right)_{\theta} d \theta d s
\end{aligned}
$$

Multiplicando (4.47) por $e^{\sigma \theta} w(\theta)$, obtemos

$$
\begin{aligned}
\frac{d}{d \theta}\left(e^{\sigma \theta}\left(w^{\prime}(\theta), w(\theta)\right)_{\theta}\right)+e^{\sigma \theta}|\nabla w(\theta)|_{\theta}^{2} \leq & (\sigma-\eta) e^{\sigma \theta}\left(w^{\prime}(\theta), w(\theta)\right)_{\theta}+e^{\sigma \theta}\left|w^{\prime}(\theta)\right|_{\theta}^{2} \\
& +e^{\sigma \theta}\left(g\left(u_{2}(\theta)\right)-g\left(u_{1}(\theta)\right), w(\theta)\right)_{\theta}
\end{aligned}
$$

Análogamente, obtemos

$$
\begin{aligned}
& \int_{\tau}^{t} \int_{s}^{t} e^{\sigma \theta}|\nabla w(\theta)|_{\theta}^{2} d \theta d s \\
& \leq \int_{\tau}^{t} e^{\sigma s}\left(w^{\prime}(s), w(s)\right)_{s} d s+(\sigma-\eta) \int_{\tau}^{t} \int_{s}^{t} e^{\sigma \theta}\left(w^{\prime}(\theta), w(\theta)\right)_{\theta} d \theta d s-(t-\tau) e^{\sigma t}\left(w^{\prime}(t), w(t)\right)_{t} \\
& \quad+\int_{\tau}^{t} \int_{s}^{t} e^{\sigma \theta}\left|w^{\prime}(\theta)\right|_{\theta}^{2} d \theta d s+\int_{\tau}^{t} \int_{s}^{t} e^{\sigma \theta}\left(g\left(u_{2}\right)-g\left(u_{1}\right), w(\theta)\right)_{\theta} d \theta d s .
\end{aligned}
$$


Substituindo (4.52) em(4.50), tomando em conta que $\sigma<\frac{\eta}{2}$, deduzimos

$$
\begin{aligned}
(t-\tau) e^{\sigma t} E_{w}(t)-\int_{\tau}^{t} e^{\sigma s} E_{w}(s) d s \leq & -\sigma(t-\tau) e^{\sigma t}\left(w^{\prime}(t), w(t)\right)_{t} \\
& +\sigma \int_{\tau}^{t} e^{\sigma s}\left(w^{\prime}(s), w(s)\right)_{s} d s \\
& +\sigma(\sigma-\eta) \int_{\tau}^{t} \int_{s}^{t} e^{\sigma \theta}\left(w^{\prime}(\theta), w(\theta)\right)_{\theta} d \theta d s \\
& +\sigma \int_{\tau}^{t} \int_{s}^{t} e^{\sigma \theta}\left(g\left(u_{2}\right)-g\left(u_{1}\right), w(\theta)\right)_{\theta} d \theta d s \\
& +\int_{\tau}^{t} \int_{s}^{t} e^{\sigma \theta}\left(g\left(u_{2}\right)-g\left(u_{1}\right), w^{\prime}(\theta)\right)_{\theta} d \theta d s .
\end{aligned}
$$

Integrando (4.51) de $\tau$ até $t$, temos

$$
\begin{aligned}
\int_{\tau}^{t} e^{\sigma \theta}|\nabla w(\theta)|_{\theta}^{2} d \theta \leq & e^{\sigma \tau}\left(w^{\prime}(\tau), w(\tau)\right)_{\tau}-e^{\sigma t}\left(w^{\prime}(t), w(t)\right)_{t}+\int_{\tau}^{t} e^{\sigma \theta}\left|w^{\prime}(\theta)\right|_{\theta}^{2} d \theta \\
& +(\sigma-\eta) \int_{\tau}^{t} e^{\sigma \theta}\left(w^{\prime}(\theta), w(\theta)\right)_{\theta} d \theta+\int_{\tau}^{t} e^{\sigma \theta}\left(g\left(u_{2}\right)-g\left(u_{1}\right), w(\theta)\right)_{\theta} d \theta .
\end{aligned}
$$

Substituindo (4.54) em (4.53), segue

$$
\begin{aligned}
(t-\tau) e^{\sigma t} E_{w}(t)+\int_{\tau}^{t} e^{\sigma \theta} E_{w}(\theta) d \theta \leq & e^{\sigma \tau}\left(w^{\prime}(\tau), w(\tau)\right)_{\tau}-(1+\sigma(t-\tau)) e^{\sigma t}\left(w^{\prime}(t), w(t)\right)_{t} \\
& +2 \int_{\tau}^{t} e^{\sigma \theta}\left|w^{\prime}(\theta)\right|_{\theta}^{2} d \theta \\
& +(2 \sigma-\eta) \int_{\tau}^{t} e^{\sigma \theta}\left(w^{\prime}(\theta), w(\theta)\right)_{\theta} d \theta \\
& +\sigma(\eta-\sigma)(1+(t-\tau) \sigma) \int_{\tau}^{t} e^{\sigma \theta}|w(\theta)|_{\theta}^{2} d \theta \\
& +\int_{\tau}^{t} e^{\sigma \theta}\left(g\left(u_{2}\right)-g\left(u_{1}\right), w(\theta)\right)_{\theta} d \theta \\
& +\sigma \int_{\tau}^{t} \int_{s}^{t} e^{\sigma \theta}\left(g\left(u_{2}\right)-g\left(u_{1}\right), w(\theta)\right)_{\theta} d \theta d s \\
& +\int_{\tau}^{t} \int_{s}^{t} e^{\sigma \theta}\left(g\left(u_{2}\right)-g\left(u_{1}\right), w^{\prime}(\theta)\right)_{\theta} d \theta d s .
\end{aligned}
$$


Integrando (4.48) de $\tau$ até $t$, resulta

$$
\begin{aligned}
e^{\sigma t} E_{w}(t)-e^{\sigma \tau} E_{w}(\tau)+\eta \int_{\tau}^{t} e^{\sigma \theta}\left|w^{\prime}(\theta)\right|_{\theta}^{2} d \theta \leq & \sigma \int_{\tau}^{t} e^{\sigma \theta} E_{\theta}(\theta) d \theta \\
& +\int_{\tau}^{t} e^{\sigma \theta}\left(g\left(u_{2}\right)-g\left(u_{1}\right), w^{\prime}(\theta)\right)_{\theta} d \theta
\end{aligned}
$$

Substituindo (4.56) em (4.55), temos

$$
\begin{aligned}
(t-\tau) e^{\sigma t} E_{w}(t) \leq & \frac{2 e^{\sigma \tau}}{\eta} E_{w}(\tau)+e^{\sigma \tau}\left(w^{\prime}(\tau), w(\tau)\right)_{\tau}-\left(1+(t-\tau) \sigma e^{\sigma t}\left(w^{\prime}(t), w(t)\right)_{t}\right. \\
& +(2 \sigma-\eta) \int_{\tau}^{t} e^{\sigma \theta}\left(w^{\prime}(\theta), w(\theta)\right)_{\theta} d \theta \\
& +\sigma(\eta-\sigma)(1+\sigma(t-\tau)) \int_{\tau}^{t}|w(\theta)|_{\theta}^{2} d \theta \\
& +\int_{\tau}^{t} e^{\sigma \theta}\left(g\left(u_{2}\right)-g\left(u_{1}\right), w(\theta)\right)_{\theta} d \theta \\
& +\sigma \int_{\tau}^{t} \int_{s}^{t} e^{\sigma \theta}\left(g\left(u_{2}\right)-g\left(u_{1}\right), w(\theta)\right)_{\theta} d \theta d s \\
& +\int_{\tau}^{t} \int_{s}^{t} e^{\sigma \theta}\left(g\left(u_{2}\right)-g\left(u_{1}\right), w^{\prime}(\theta)\right)_{\theta} d \theta d s \\
& +\frac{2}{\eta} \int_{\tau}^{t} e^{\sigma \theta}\left(g\left(u_{2}\right)-g\left(u_{1}\right), w^{\prime}(\theta)\right)_{\theta} d \theta
\end{aligned}
$$

Pelas propriedades de $g$, desigualdade de Hölder e os teoremas de imersão, obtemos

$$
\begin{aligned}
& \int_{\tau}^{t} \int_{s}^{t} e^{\sigma \theta}\left(g\left(u_{2}\right)-g\left(u_{1}\right), w(\theta)\right)_{\theta} d \theta d s \\
& \leq C(t-\tau)\left(\int_{\tau}^{t} e^{\sigma \theta} \int_{\mathscr{O}_{\theta}}\left(1+\left|u_{1}(\theta)\right|^{\frac{2 N}{N-2}}+\left|u_{2}(\theta)\right|^{\frac{2 N}{N-2}}\right) d x d \theta\right)^{\frac{1}{2}}\left(\int_{\tau}^{t} e^{\sigma \theta}|w(\theta)|_{\theta}^{2}\right)^{\frac{1}{2}} \\
& \leq C(t-\tau)\left(e^{\sigma t}+\left(\int_{\tau}^{t} e^{\sigma \theta}\left(\left|\nabla u_{1}(\theta)\right|_{\theta}^{2}+\left|\nabla u_{2}(\theta)\right|_{\theta}^{2}\right) d \theta\right)^{\frac{N}{N-2}}\right)^{\frac{1}{2}}\left(\int_{\tau}^{t} e^{\sigma \theta}|w(\theta)|_{\theta}^{2}\right)^{\frac{1}{2}}
\end{aligned}
$$

Como estamos interessados em o comportamento das soluções quando $\tau \longrightarrow-\infty$ de $(4.28)$, deduzimos que

$$
\left(\int_{\tau}^{t} e^{\sigma \theta}|\nabla u(\theta)|_{\theta}^{2} d \theta\right)^{\frac{N}{N-2}} \leq C_{t, \tau}<\infty \text { para todo }\left(u_{\tau 0}, u_{\tau 1}\right) \in D(\tau)
$$


Combinando (4.58) e (4.59), obtemos

$$
\int_{\tau}^{t} \int_{s}^{t} e^{\sigma \theta}\left(g\left(u_{2}\right)-g\left(u_{1}\right), w(\theta)\right)_{\theta} d \theta d s \leq \widehat{C_{t, \tau}}\left(\int_{\tau}^{t} e^{\sigma \theta}|w(\theta)|_{\theta}^{2}\right)^{\frac{1}{2}}<\infty, \quad \forall\left(u_{\tau 0}^{i}, u_{\tau 1}^{i}\right) \in D(\tau)
$$

Denotemos por $\Phi_{\tau}$ à função definida sobre $\widehat{B}$, dado por

$$
\begin{aligned}
\frac{1}{2} \Phi_{\tau}\left(\left(u_{0 \tau}^{1}, u_{1 \tau}^{1}\right),\left(u_{0 \tau}^{2}, u_{1 \tau}^{2}\right)\right)= & \widehat{C_{t, \tau}} e^{-\sigma t}\left(1+(t-\tau)^{-1}\right)\left(\int_{\tau}^{t} e^{\sigma \theta}|w(\theta)|_{\theta}^{2}\right)^{\frac{1}{2}} \\
& +\sigma(\eta-\sigma)\left(\sigma+2(t-\tau)^{-1}\right) e^{-\sigma t} \int_{\tau}^{t} e^{\sigma \theta}|w(\theta)|_{\theta}^{2} d \theta \\
& +(t-\tau)^{-1} e^{-\sigma t} \int_{\tau}^{t} \int_{s}^{t} e^{\sigma \theta}\left(g\left(u_{2}\right)-g\left(u_{1}\right), w^{\prime}(\theta)\right)_{\theta} d \theta d s \\
& -\left(\sigma+(t-\tau)^{-1}\right)\left(w^{\prime}(t), w(t)\right)_{t} \\
& +2 \eta^{-1}(t-\tau)^{-1} e^{-\sigma t} \int_{\tau}^{t} e^{\sigma \theta}\left(g\left(u_{2}\right)-g\left(u_{1}\right), w^{\prime}(\theta)\right)_{\theta} d \theta
\end{aligned}
$$

Então, de (4.57), (4.60) e (4.61), resulta

$$
E_{w}(t) \leq C_{\tau, t}^{\prime}(t-\tau)^{-1} e^{\sigma \tau} E_{w}(\tau)+\frac{1}{2} \Phi_{\tau}\left(\left(u_{0 \tau}^{1}, u_{1 \tau}^{1}\right),\left(u_{0 \tau}^{2}, u_{1 \tau}^{2}\right)\right)
$$

Daí, temos

$$
E_{w}(t) \leq \frac{1}{2} C_{\tau, t}^{\prime}(t-\tau)^{-1} e^{\sigma \tau}\left(|\nabla w(\tau)|_{\tau}^{2}+\left|w^{\prime}(\tau)\right|_{\tau}^{2}\right)+\frac{1}{2} \Phi_{\tau}\left(\left(u_{0 \tau}^{1}, u_{1 \tau}^{1}\right),\left(u_{0 \tau}^{2}, u_{1 \tau}^{2}\right)\right)
$$

Como $\left(u_{0 \tau}^{1}, u_{1 \tau}^{1}\right)$ e $\left(u_{0 \tau}^{2}, u_{1 \tau}^{2}\right)$ pertencem a $\widehat{D}$, existe $\rho_{\widehat{D}} \in R_{\sigma}$ tal que

$$
|\nabla w(t)|_{t}^{2}+\left|w^{\prime}(t)\right|_{t}^{2} \leq C_{\tau, t}^{\prime}(t-\tau)^{-1} e^{\sigma \tau} \rho_{\widehat{D}}^{2}(\tau)+\Phi_{\tau}\left(\left(u_{0 \tau}^{1}, u_{1 \tau}^{1}\right),\left(u_{0 \tau}^{2}, u_{1 \tau}^{2}\right)\right)
$$

Portanto, da propriedade de $\mathscr{D}_{\sigma}$, dado qualquer $\varepsilon>0$, existe $\tau_{0}=\tau_{0}(\varepsilon, t, \widehat{D})$ tal que

$$
\| U(t, \tau)\left(u_{\tau 0}^{1}, u_{\tau 1}^{1}\right)-U(t, \tau)\left(u_{\tau 0}^{2}, u_{\tau 1}^{2}\right)||_{X_{t}}=|\nabla w(t)|_{t}^{2}+\left|w^{\prime}(t)\right|_{t}^{2} \leq \varepsilon+\Phi_{\tau}\left(\left(u_{0 \tau}^{1}, u_{1 \tau}^{1}\right),\left(u_{0 \tau}^{2}, u_{1 \tau}^{2}\right)\right)
$$


para todo $\left(u_{0 \tau}^{1}, u_{1 \tau}^{1}\right),\left(u_{0 \tau}^{2}, u_{1 \tau}^{2}\right) \in B(\tau), \operatorname{com} \tau \leq \tau_{0}$.

Para aplicar o teorema 4.4.2 resta mostrar que $\Phi_{\tau}$ definida por (4.61) é contrativa, isto é, existe $\tau_{0} \leq t$ tal que, se $\tau \leq \tau_{0}$, dado qualquer sequência $\left\{\left(u_{\tau 0}^{n}, u_{\tau 1}^{n}\right)\right\}$ de $B(\tau)$ existe uma subsequência $\left\{\left(u_{\tau 0}^{n_{k}}, u_{\tau 1}^{n_{k}}\right)\right\}$ tal que

$$
\lim _{k \rightarrow \infty} \lim _{l \rightarrow \infty} \Phi_{\tau}\left(\left(u_{0 \tau}^{n_{k}}\right), u_{1 \tau}^{n_{k}},\left(u_{0 \tau}^{n_{l}}, u_{1 \tau}^{n_{l}}\right)\right)=0
$$

\section{Afirmação: A aplicação $\Phi_{\tau}$ é contrativa}

Seja $u_{n}$ as soluções do problema (3.1) correspondente aos dados $\left(u_{0 \tau}^{n}, u_{1 \tau}^{n}\right) \in B(\tau)$. De (4.28), temos

$$
\left|\nabla u_{n}(s)\right|_{s}^{2}+\left|u_{n}^{\prime}(s)\right|_{s}^{2} \leq C_{1} e^{-\sigma s} e^{\sigma \tau}\left(\rho_{\widehat{D}}^{2}(\tau)+1\right)+C_{2} e^{-\sigma s} \int_{\tau}^{s} e^{\sigma \theta}\|f(\theta)\|_{H^{-1}\left(\mathscr{O}_{\theta}\right)} d \theta+C_{3}
$$

para todo $s \in[\tau, t]$ e $n \in \mathbb{N}$.

Como $e^{\sigma \tau} \rho_{\widehat{D}}^{2}(\tau) \rightarrow 0$ quando $\tau \rightarrow-\infty$ segue que existe $\tau_{0} \leq t$, tal que para todo $\tau \leq \tau_{0}$, tem-se

$$
\left|\nabla u_{n}(s)\right|_{s}^{2}+\left|u_{n}^{\prime}(s)\right|_{s}^{2} \leq C_{t, \tau}<+\infty \text { para todo } s \in[\tau, t] \text { e } n \in \mathbb{N}
$$

Então, sem perda de generalidade, assumimos que

$$
\begin{aligned}
& u_{n} \rightarrow u \text { fraco estrela em } L^{\infty}\left(\tau, t ; H_{0}^{1}\left(\mathscr{O}_{s}\right)\right), \\
& u_{n}^{\prime} \rightarrow u^{\prime} \text { fraco estrela em } L^{\infty}\left(\tau, t ; L^{2}\left(\mathscr{O}_{s}\right)\right)
\end{aligned}
$$

Então pelos teoremas de imersão, temos

$$
u_{n} \rightarrow u \text { fortemente em } C\left([\tau, t] ; L^{2}\left(\mathscr{O}_{s}\right)\right)
$$

Por outro lado, de acordo com [15], Lemma 8.1, p.275, e (4.66) tem-se que a sequência $\left\{u_{n}\right\}$ é 
limitada em $C_{b}\left(\tau, t ; H_{0}^{1}\left(\mathscr{O}_{s}\right)\right)$ e conseqüentemente a sequência $\left\{u_{n}(s)\right\}$ é limitado em $H_{0}^{1}\left(\mathscr{O}_{s}\right)$ para cada $s \in[\tau, t]$. Assim, de (4.67), temos

$$
u_{n}(s) \rightarrow u(s) \text { fracamente em } H_{0}^{1}\left(\mathscr{O}_{s}\right)
$$

de acordo com os teoremas de imersão compacta, concluimos que

$$
G\left(u_{n}(s)\right) \rightarrow G(u(s)) \text { fortemente em } L^{1}\left(\mathscr{O}_{s}\right), \text { para todo } s \in[\tau, t]
$$

Por outro lado, pela imersão contínua $H_{0}^{1}\left(\mathscr{O}_{S}\right) \hookrightarrow L^{\frac{2 N}{N-2}}\left(\mathscr{O}_{S}\right)$, e as imersões compactas $H_{0}^{1}\left(\mathscr{O}_{S}\right) \hookrightarrow$ $L^{2}\left(\mathscr{O}_{S}\right), H_{0}^{1}\left(\mathscr{O}_{S}\right) \hookrightarrow L^{\frac{2 N-2}{N-2}}$, deduzimos que

$$
\begin{gathered}
u_{n} \rightarrow u \text { fraco estrela em } L^{\infty}\left(\tau, t ; L^{\frac{2 N}{N-2}}\left(\mathscr{O}_{S}\right)\right), \\
u_{n} \rightarrow u \text { em } L^{2}\left(\tau, t ; L^{2}\left(\mathscr{O}_{s}\right)\right), \\
u_{n}(\tau) \rightarrow u(\tau) \text { e } u_{n}(t) \rightarrow u(t) \text { em } L^{2}\left(\mathscr{O}_{\tau}\right), L^{2}\left(\mathscr{O}_{t}\right) \text { e } L^{\frac{2 N-2}{N-2}}\left(\mathscr{O}_{\tau}\right), L^{\frac{2 N-2}{N-2}}\left(\mathscr{O}_{t}\right) .
\end{gathered}
$$

\section{Agora, nós vamos lidar com os termos em (4.61), um por um:}

1. Em primeiro lugar, a partir de (4.71), segue

$$
\lim _{n \rightarrow+\infty} \lim _{m \rightarrow+\infty} \int_{\tau}^{t}\left|u_{n}(\theta)-u_{m}(\theta)\right|_{\theta}^{2} d \theta=0
$$

2. De (4.72), tem-se

$$
\lim _{n \rightarrow+\infty} \lim _{m \rightarrow+\infty} \int_{\mathscr{O}_{t}}\left(u_{n}^{\prime}(t)-u_{m}^{\prime}(t)\right)\left(u_{n}(t)-u_{m}(t)\right) d x=0
$$


3. Por outro lado,

$$
\begin{aligned}
& \int_{\tau}^{t} e^{\sigma \theta} \int_{\mathscr{O}_{\theta}}\left(u_{n}^{\prime}(\theta)-u_{m}^{\prime}(\theta)\right)\left(g\left(u_{n}(\theta)\right)-g\left(u_{m}(\theta)\right)\right) d x d \theta \\
& =\int_{\tau}^{t} e^{\sigma \theta} \int_{\mathscr{O}_{\theta}} u_{n}^{\prime}(\theta) g\left(u_{n}(\theta)\right) d x d \theta+\int_{\tau}^{t} e^{\sigma \theta} \int_{\mathscr{O}_{\theta}} u_{m}^{\prime}(\theta) g\left(u_{m}(\theta)\right) d x d \theta \\
& \quad-\int_{\tau}^{t} e^{\sigma \theta} \int_{\mathscr{O}_{\theta}} u_{n}^{\prime}(\theta) g\left(u_{m}(\theta)\right) d x d \theta-\int_{\tau}^{t} e^{\sigma \theta} \int_{\mathscr{O}_{\theta}} u_{m}^{\prime}(\theta) g\left(u_{n}(\theta)\right) d x d \theta \\
& =e^{\sigma t} \int_{\mathscr{O}_{t}} G\left(u_{n}(t)\right) d x-e^{\sigma \tau} \int_{\mathscr{O}_{\tau}} G\left(u_{n}(\tau)\right) d x \\
& \quad+e^{\sigma t} \int_{\mathscr{O}_{t}} G\left(u_{m}(t)\right) d x-e^{\sigma \tau} \int_{\mathscr{O}_{\theta}} G\left(u_{m}(\tau)\right) d x \\
& \quad-\int_{\tau}^{t} e^{\sigma \theta} \int_{\mathscr{O}_{\theta}} u_{n}^{\prime}(\theta) g\left(u_{m}(\theta)\right) d x d \theta-\int_{\tau}^{t} e^{\sigma \theta} \int_{\mathscr{O}_{\theta}} u_{m}^{\prime}(\theta) g\left(u_{n}(\theta)\right) d x d \theta \\
& \quad-\sigma \int_{\tau}^{t} e^{\sigma \theta} \int_{\mathscr{O}_{\theta}} G\left(u_{n}(\theta)\right) d x d \theta-\sigma \int_{\tau}^{t} e^{\sigma \theta} \int_{\mathscr{O}_{\theta}} G\left(u_{m}(\theta)\right) d x d \theta .
\end{aligned}
$$

Fazendo $m, n \rightarrow+\infty$ em (4.75), de (4.69), obtemos

$$
\begin{aligned}
& \lim _{n \longrightarrow+\infty m \longrightarrow+\infty} \lim _{\tau} e^{\sigma \theta} \int_{\mathscr{O}_{\theta}}\left(u_{n}^{\prime}(\theta)-u_{m}^{\prime}(\theta)\right)\left(g\left(u_{n}(\theta)-g\left(u_{m}(\theta)\right)\right) d x d \theta\right. \\
&=e^{\sigma t} \int_{\mathscr{O}_{t}} G(u(t)) d x-e^{\sigma \tau} \int_{\mathscr{O}_{\tau}} G(u(\tau)) d x \\
&+e^{\sigma t} \int_{\mathscr{O}_{t}} G(u(t)) d x-e^{\sigma \tau} \int_{\mathscr{O}_{\theta}} G(u(\tau)) d x \\
&-\int_{\tau}^{t} e^{\sigma \theta} \int_{\mathscr{O}_{\theta}} u^{\prime}(\theta) g(u(\theta)) d x d \theta-\int_{\tau}^{t} e^{\sigma \theta} \int_{\mathscr{O}_{\theta}} u^{\prime}(\theta) g(u(\theta)) d x d \theta \\
&-\sigma \lim _{n \longrightarrow+\infty} \int_{\tau}^{t} e^{\sigma \theta} \int_{\mathscr{O}_{\theta}} G\left(u_{n}(\theta)\right) d x d \theta-\sigma \lim _{m \longrightarrow+\infty} \int_{\tau}^{t} e^{\sigma \theta} \int_{\mathscr{O}_{\theta}} G\left(u_{m}(\theta)\right) d x d \theta \\
&= \sigma \lim _{n \longrightarrow \infty} \int_{\tau}^{t} e^{\sigma \theta} \int_{\mathscr{O}_{\theta}}\left(G(u(\theta))-G\left(u_{n}(\theta)\right)\right) d x d \theta \\
&+\sigma \lim _{m \longrightarrow \infty} \int_{\tau}^{t} e^{\sigma \theta} \int_{\mathscr{O}_{\theta}}\left(G(u(\theta))-G\left(u_{m}(\theta)\right)\right) d x d \theta \\
&= 0 .
\end{aligned}
$$

4. Da mesma forma, uma vez que $\left|\int_{s}^{t} e^{\sigma \theta} \int_{\mathscr{O}_{\theta}}\left(u_{n}^{\prime}(\theta)-u_{m}^{\prime}(\theta)\right)\left(g\left(u_{n}(\theta)\right)-g\left(u_{m}(\theta)\right)\right) d x d \theta d s\right|$ 
é limitado para cada $s \in[\tau, t]$, então por (4.75) e o teorema da convergência dominada, obtemos

$$
\begin{aligned}
& \lim _{n \rightarrow+\infty} \lim _{m \rightarrow+\infty} \int_{\tau}^{t} \int_{s}^{t} e^{\sigma \theta} \int_{\mathscr{O}_{\theta}}\left(u_{n}^{\prime}(\theta)-u_{m}^{\prime}(\theta)\right)\left(g\left(u_{n}(\theta)\right)-g\left(u_{m}(\theta)\right)\right) d x d \theta d s \\
& \quad=\int_{\tau}^{t}\left(\lim _{n \rightarrow+\infty} \lim _{m \rightarrow+\infty} \int_{s}^{t} e^{\sigma \theta} \int_{\mathscr{O}_{\theta}}\left(u_{n}^{\prime}(\theta)-u_{m}^{\prime}(\theta)\right)\left(g\left(u_{n}(\theta)\right)-g\left(u_{m}(\theta)\right)\right) d x d \theta\right) d s \\
& \quad=\int_{\tau}^{t} 0 d s=0 .
\end{aligned}
$$

Combinando (4.73) -(4.76), sabemos que $\Phi_{\tau}(\cdot, \cdot)$ é uma função contrativa em $B(\tau) \times B(\tau)$. 


\section{Referências Bibliográficas}

[1] Bardos, C. \& Chen, G.; Control and stabilization for the wave equation. III. Domain with moving boundary, SIAM J. Control Optim. 19 (1981) 123-138.

[2] Brézis, H.; Opérateurs Maximaux Monotones et Semi-Groupes de Contractions dans les Espaces de Hilbert; North-Holland. Mathematics Studies (1973).

[3] Brézis, H.; Analyse Fonctionnelle, Théorie et Applications; Masson, Paris (1983).

[4] Caraballo, T.; Lukaszewics, G. \& Real, J.; Pullabck attractor for asymptotically compact non-autonomous dynamical system; Nonlinear Analysis. 64 (2006) 484-498.

[5] Caraballo, T.; Lukaszewics, G. \& Real, J.; Pullback attractors for non-autonomous 2DNavier-Stokes equations in some unbounded domains; Comptes Rendus Mathématique. 342 (2006) 263-268.

[6] Carvalho, A. N.; Langa, J. \& Robinson, J.; Attractors for Infinite-Dimensional NonAutonomous Dynamical Systems; Applied Mathematical Sciences. Springer, New York 182 (2013).

[7] Chepyzhov, V. V. \& Vishik, M.I.; Attractors of Equations of Mathematical Physics; American Mathematical Society (2002).

[8] Coddington, E. A. \& Levinson, N.; Theory of Ordinary Differential Equations; New York. McGraw-Hill (1955).

[9] Cooper, J. \& Bardos, C.; A nonlinear wave equation in a time dependent domain, J. Math. Anal. Appl. 42 (1973) 29-60.

[10] García-Luengo, J.; Marín-Rubio, P. \& Real, J.; Pullback attractors in V for non-autonomous $2 D$-Navier-Stokes equations and their tempered behaviour; Journal of Differential Equations. 252 (2012) 4333-4356.

[11] Ha, Tae Gab \& Park, Jong Yeoul; Global existence and uniform decay of a damped KleinGordon equation in a noncylindrical domain; Nonlinear Analysis. 74 (2011) 577-584.

[12] Kloeden, P. E.; Marín-Rubio, P. \& Real, J.; Pullback attractors for a semilinear heat equation in a non-cylindrical domain; Journal of Differential Equations. 244 (2008) 2062-2090. 
[13] Kloeden, P. E.; Real, J. \& Sun, C.; Pullback attractors for a semilinear heat equation on time-varying domains; Journal of Differential Equations. 246 (2009) 4702-4730.

[14] Lions, J. L.; Une remarque sur les problèmes d'evolution non Linéaires dans des domaines non cylindriques; Rev. Romaine Math. Pure Appl. 9 (1964) 11-18.

[15] Lions, J. L.; Magenes, E.; Problèmes aux Limites non Homogènes; Dunod, Paris (1968).

[16] Lions, J. L.; Quelques Méthodes de Résolution des Problèmes aux Limites non Linéaries; Dunod Gauthier-Villars, Paris, (1969).

[17] Medeiros, L. A.; Non-linear wave equations in domains with variable boundary; Archive for Rational Mechanics and Analysis 47 (1972) 47-58.

[18] Medeiros, L. A.; Limaco, J. \& Menezes, S. B.; Vibrations of elastic strings: mathematical aspects. I.; Journal Computational Analysis and Applications. 4 (2002) 91-127.

[19] Medeiros, L. A.; Limaco, J. \& Menezes, S. B.; Vibrations of elastic strings: mathematical aspects. II.; Journal of Computational Analysis Applications. 4 (2002) 211-263.

[20] Sun, C.; Cao, D. \& Duan, J.; Non-autonomous dynamics of wave equations with nonlinear damping and critical nonlinearity; Nonlinearity. 19 (2006) 2645-2665.

[21] Temam, R.; Navier Stokes Equations, Theory and Numerical Analysis; North Holland, Amsterdam; (1979).

[22] Wang, Y.; On the upper semicontinuity of pullback attractors with applications to plate equations; Communications on Pure and Applied Analysis. 9 (2010) 1653-1673.

[23] Yan, X. \& Zhong, C.; On pullback attractor for non-autonomous weakly dissipative wave equations; Dynamical Systems. An International Journal. 24 (2009) 97-108.

[24] A. V. Babin and M. I. Vishik, Attractors of Evolution Equations, Studies in Mathematics and its Application 25, North-Holland, Amsterdam, 1992.

[25] Conti, M.; Pata, V. \& Temam, R.; Attractors for processes on time-dependent spaces. Applications to wave equations, J. DifferentialEquations 255 (2013) 1254-1277.

[26] J. K. Hale, Asymptotic behavior of dissipative systems, Mathematical Surveys and Monographs, 25. American Mathematical Society, Providence, RI, 1988.

[27] O. Ladyzhenskaya, Attractors for Semi-groups and Evolution Equations, Cambridge University Press, 1991.

[28] R. Temam, Infinite-Dimensional Dynamical Systems in Mechanics and Physics, Applied Mathematical Sciences 68, Springer-Verlag, New York, 1988. 